M A

MW MM

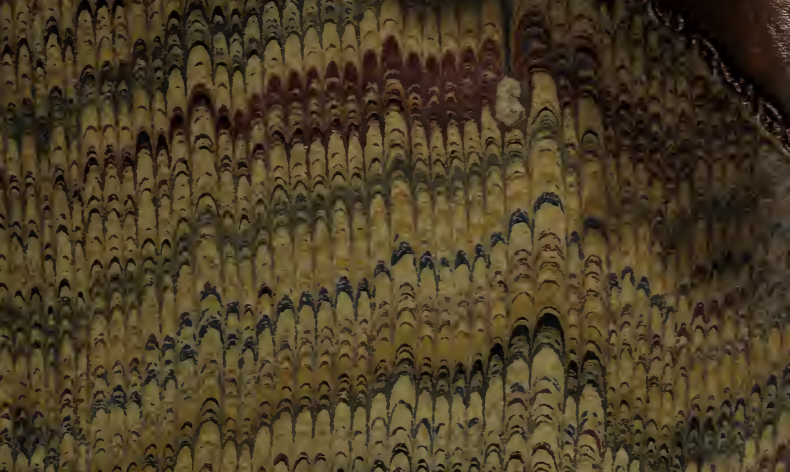

Con

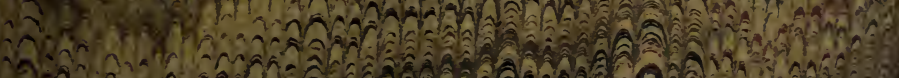

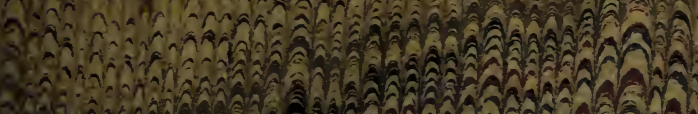

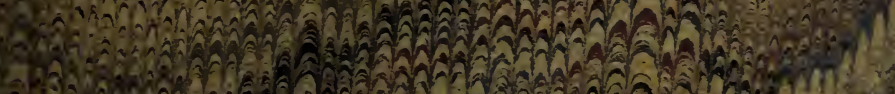

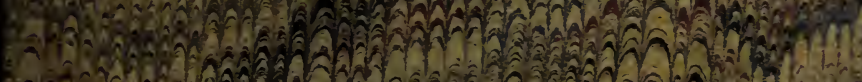
A Afa

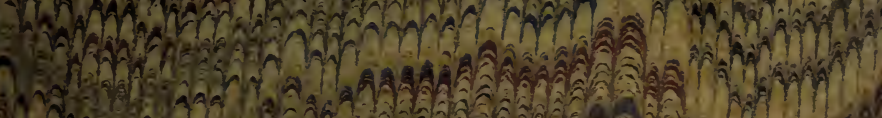

Mnh

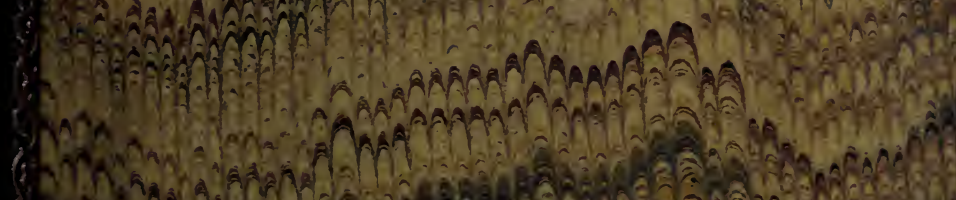

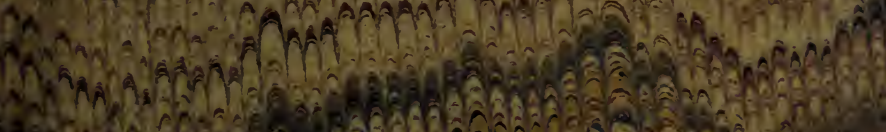

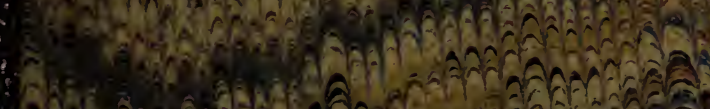
- Now

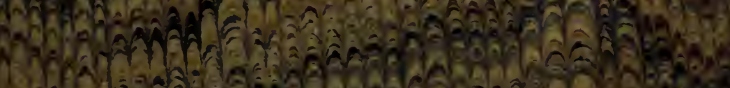

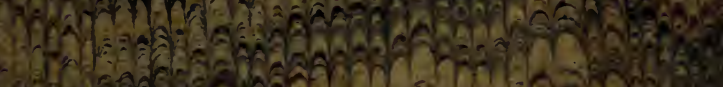

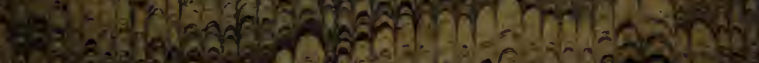

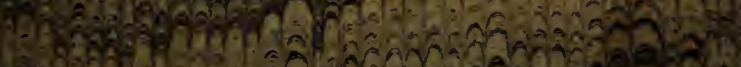




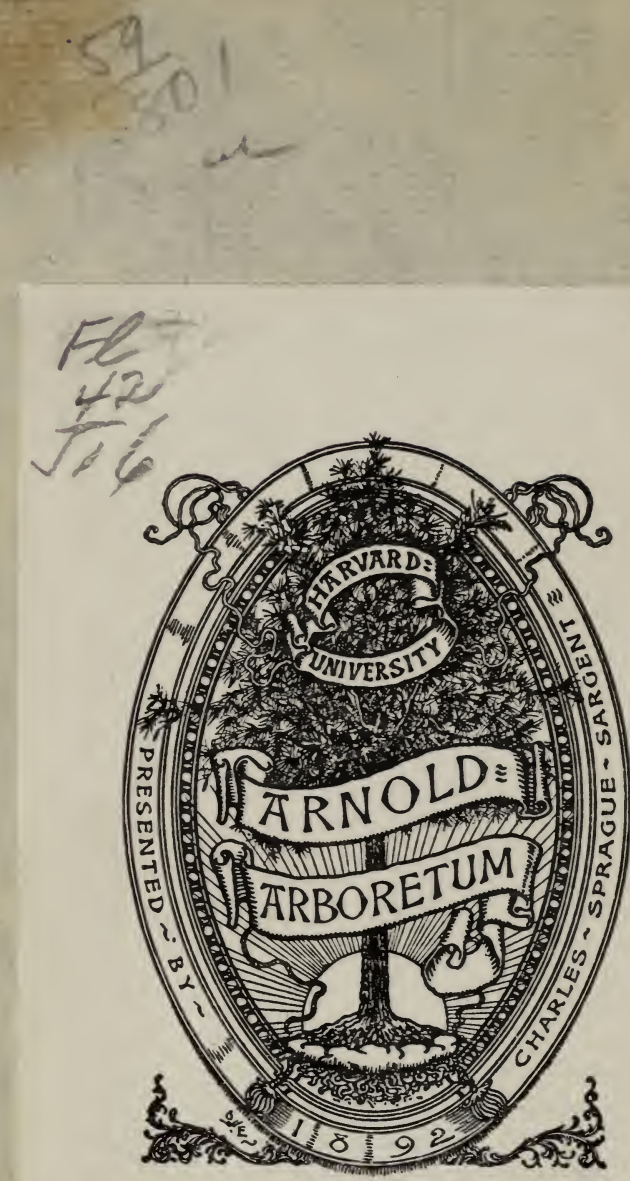




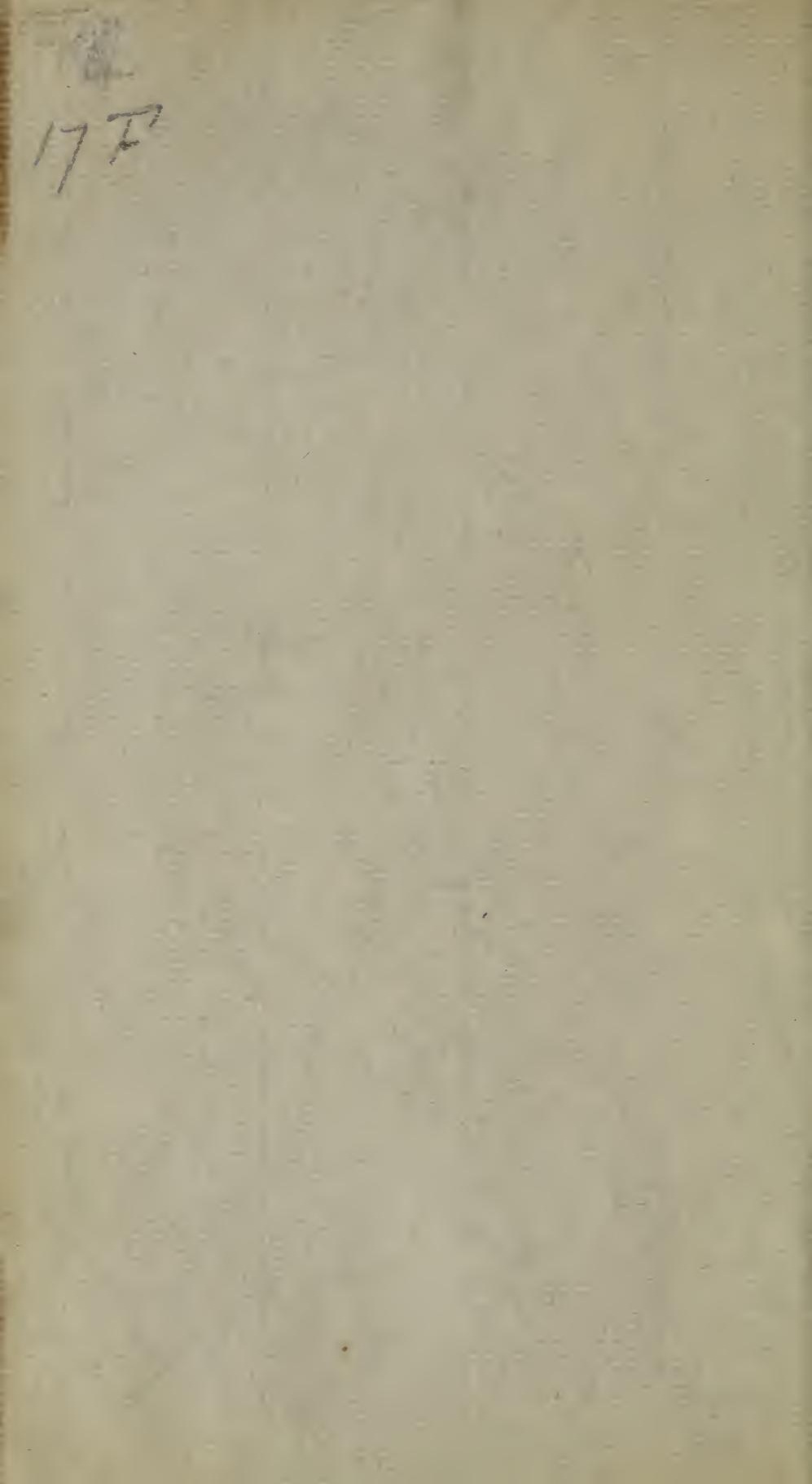



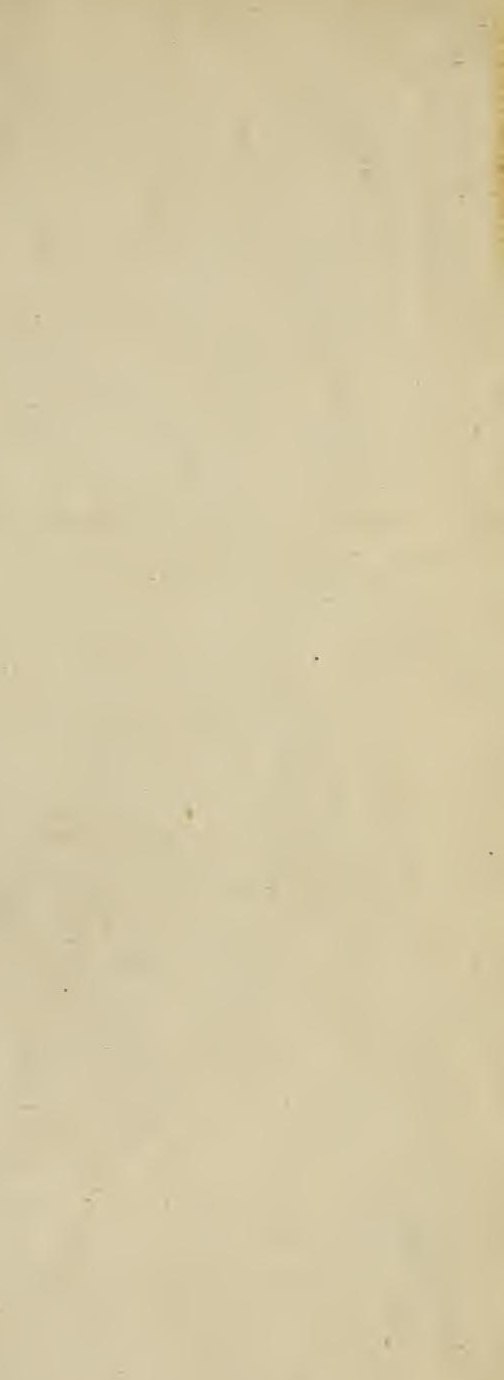

I 


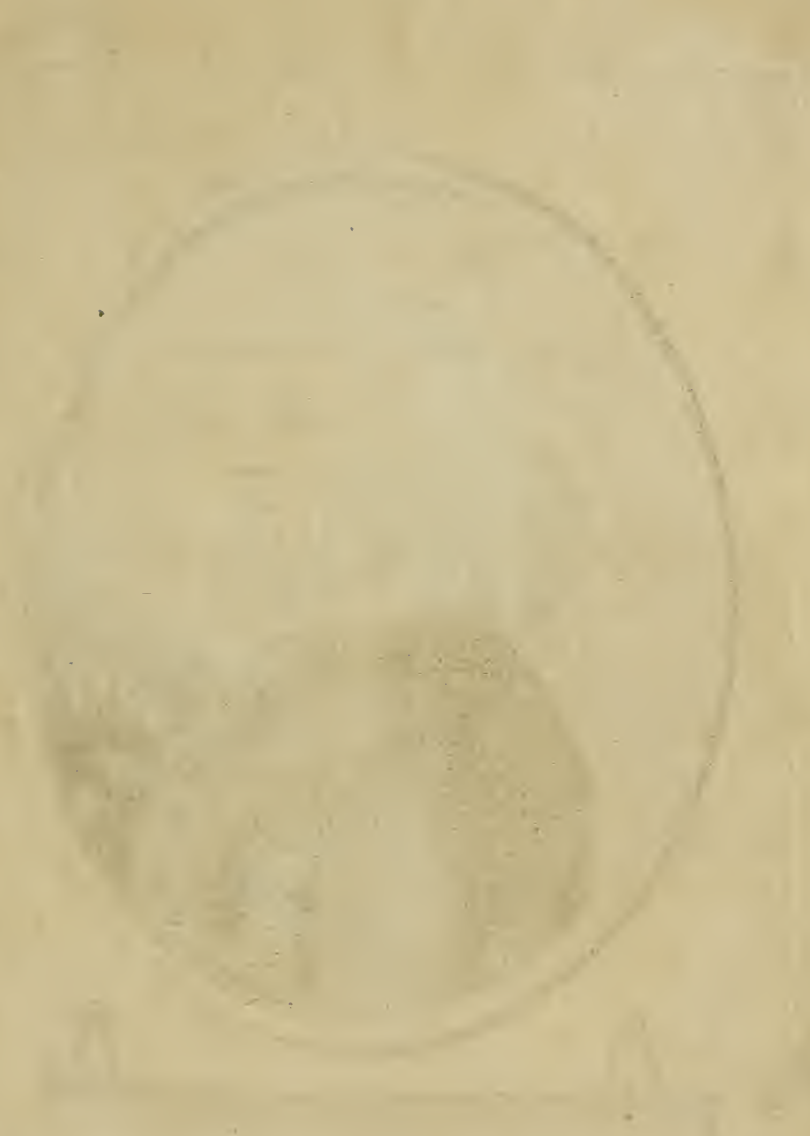

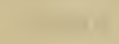
$-\infty+2=$ ta

$\div \quad+10$ 


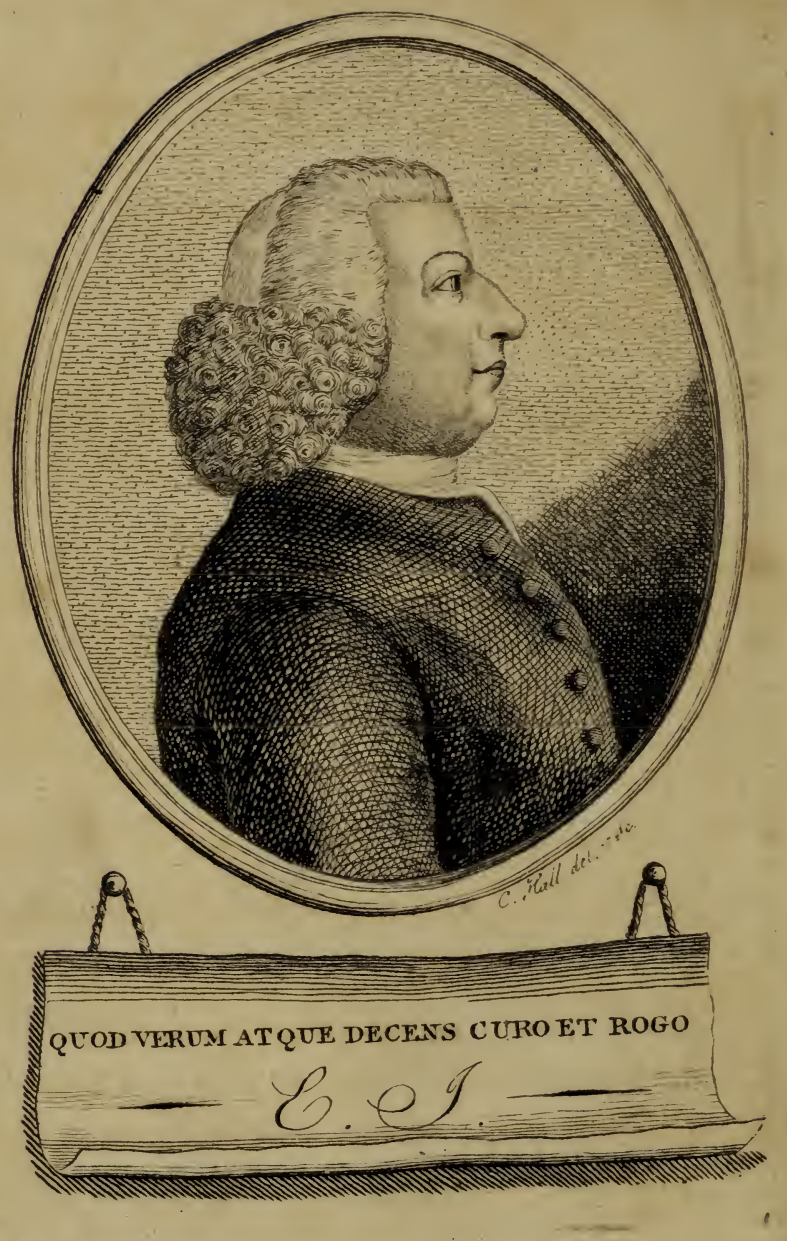


PLANTE FAVERSHAMIENSES.

\section{A

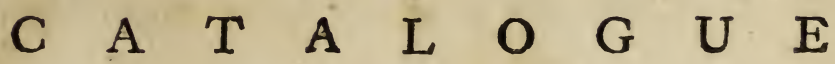 OF THE} MORE PERFECT
$\mathbf{P}$
L
A
N
$T$
$\mathbf{S}$

Growing fpontaneoufly about

$\begin{array}{lllllllll}\text { F } & A & V & E & R & S & H & A & M \text {, }\end{array}$ I N T H E

C O U N T Y OF K E N T. W IT H A N

$\begin{array}{llllllll}\text { A } & \mathrm{P} & \mathrm{P} & \mathrm{E} & \mathrm{N} & \mathrm{D} & \mathrm{I} & \mathrm{X}\end{array}$ Exhibiting a SHORT VIEW of the

F O S S I L B O D I E S OF THE ADJACEN T

I S L A N D OF S H E P E Y. By E D W A R D J A C O B, Efq; F.S.A.

Confder the Lillies of the Field borv they grow - Matt. vii. 28 .

$$
\text { L } O \quad N \quad D \text { O N : }
$$

Printed for the Author; by J. MAR ch, on Tower-Hill. Sold by B. WHITE, at Horace's-Head, FleEt-Street; and T. EVANS, PAter-Noster-Row. 
Wow: $19 / 2$ $27>59$ 


\section{T $\mathrm{O}$}

\section{Mr. JOH N WH I T E,}

MERCHANT OF LONDON,

IN A GRATEFUL SENSE OF HIS

LONG AND SINGULAR

$\begin{array}{lllllllllll}F & R & I & E & N & D & S & H & \text { I } & \text { P. }\end{array}$

THIS LITTLE WORK

IS MOST RESPECTFULLY INSCRIBED

BY HIS EVER OBIIGED,

AND VERY HUMBLE SERVANT,

\section{EDWARD JACOB.}

Faverham, Aug. 10, 1777 . 
Nefcio quid fafcincrerit nofros ub loba mens traikatur ad peregrinas picmes, ille vero quas pedibus conculcomus, omino relinquuriur.

C. L.ink a i Bib!. Botan p. 24. 


\section{$P \quad R \quad E \quad F \quad A \quad C \quad E$.}

$\mathcal{T}^{\prime} \mathrm{E}$ enfuing Collection of $P L A N \mathcal{T} S$ was begun many Years fince upon the Bafis of the late Rev. Mr. Bateman's Catalogue, with whom, in the early part of Life, the Autbor made feveral Botanical Excurfions bereabouts; and it hatb gradually kept increafing ever fince, as the conftant Vifits, in the way of his Profefion, to different Parts, gave bim opportunities of fearching after them. It weas ever intended for bis own private Ufe and infructive Entertainment, till lately, at the recommiendation of feveral Gentlemen well verfed in Botany, it bath been revifed and digefled, and is now offered to the Lovers of that Science, as a means, however finall, of promoting the topicil Knowledge of Englinh Plants; if it flall prove agreable to their. Taffe, be woill think binglif amply rewarded for his Pains.

The great Promoter of its publieation was the Late woorthy Richard Warner, Efg; of Woodford-Row, in Efiex, who not only permiteed the 


\section{$\begin{array}{lllllll}P & R & E & F & A & C & E .\end{array}$}

free Ufe of bis Plantæ Woodfordienfes, (without the Aid of wbich, and Mr. Hudfon's excellent Flora Anglica, it would never bave been undertaken) but alfo afifted the Autbor in the Profecution of it, and above all, introduced bim to the acquaintance of that accurate Botanift, Mr. Alchorne, AfjayMafter of the Mint, whofe kind Informations and Corrections bave been of the greateft Advantage.

The principal Inducement for the Publication was to rectify the Account given in Mr. Blackfton's Specimen Botanicum, taken from $M r$. Bateman's M.S. Catalogue of near Two Hundred Plants growing about this Town, becaufe many of the rare ones at this time, are not to be found in the Places therein referred to, which bave of late been often diligently fearched in bope of retrieving them, as they would bave added much to this Work, but in vain.

In the Courje of about Seventy Years fince that Catalogue was made, mony Caufes and Accidents may be afigned for the lofs of fome of the rare Plants there mentioned, yet none greater than the indufrious band of improving Hufbandry. $M r$. Bateman might pofibly bave been miftaken in others, 


\section{$\begin{array}{lllllll}P & R & E & F & A & C & E\end{array}$}

otbers, and pofibly too fome may be overlooked by the Autbor, who is not fo conceited as to tbink be bath difcovered all the Plants growing round bim. Those Gentlemen who are fond of the Study muft be Jenfible, that a fingle Perfon, whofe neceflary attention in the different Branches of bis Profefion was fully engaged, could not afford fufficient Leifure, bowever fervent bis Inclination might be, to fearch at proper Seafons every corner of a Diftrict Five or Six Miles Diameter, when even the great $M r$. Ray could make confiderable. Additions to the firf Catalogue of bis Cambridgerhire Plants, and yet leave many unobferved, to be added thereto by Dr. Martyn, and others.

In order, bowever, to Jupply bis Deficiencies, the Autbor bath inferted fome fero rare Plants difcovered at a greater diftance in the Eaftern parts of the County, either by bimfelf, or communicated to bim by bis obliging Friends, $M r$. Alchorne, the Rev. Mr. Jones, of Pluckley, and $M r$. Boys of Sandwich, for wobich Favours be begs they will be pleafed to accept bis moft grateful Acknotuledgements. 


\section{$P \quad R \quad E A$ C E.}

As be batb compoled bis Catalogue upon $M r$. Warner's alphabetical Plan, be bopes the Preference given to Mr. Ray's Names will be pardoned by the more fcientific Botanifts, especially as be bath printed an Index of thofe of Linnæus.

The Appendix, giving a Sort Account of the Shepey Foljils, it is prefumed will not be deemed an improper Addition, by the Lovers of Natural Hiflory, as bitberlo no particular one batb been offered to their Notice, altbough fo well deferving of it.

The Plate of the Author is the unexpected Donation iof an obliging Friend.

The A B BREVIA T ION S explained.

R. S. J. Raii Synopfis Stirpium Britannicarum. Ed. III. 1724 .

H. F. Gul. Hudfoni Flora Anglica. 1762 . A. Annual. B. Biennial. P. Perennial. S. Shrub, or Tree.

The Month (as March, \&rc.) denotes the Time of the Plants flowering.

* This Mark placed before a Plant, although found wild, thews it moft probably to have originated an. from Seed difperfed by Birds, or otherwife from Gardens. 


\section{( I )}

PLANTÆFAVERSHAMIENSES.

A

A BSINTHIUM marinum album. R. S. 188 :

A ARTEMISIA maritima. H.F. วิr White Sea-Wormwood.

In the Salt Marßhes-very common. Augurt. P.

ABSINTHIUM vulgare. R. S. 188.

ARTEMISIA Abfintbium. H.F. 31 .

Common Wormwood.

In Farm-yards and on dry banks-common. Augurt. P.

* ACER majus. R. S. 407.

ACER PSeudo-Platanus. H.F. 37 s.

The greater Maple or Sycamore Tree.

In a Hedge near Brenly and Hannets Forital -- very uncommon. May. S.

ACER minus. R.S. 470 .

ACER compeftre. H. F. 379:

The Common Mapie.

In Hedges - rery commoiz. May S.

B 


\section{(2)}

ACINOS. R. S. 238.

THYMUS Acinos. H. F. 230.

Wild Bafil.

On Badgen Dorons - plentifully. July. A.

ADIANTUM nigrum Officinarum. R.S. 126 . ASPLENIUM Adiantum-nigrum. H.F. 386.

Common black Maiden-hair.

On the bollow fandy Banks in Byfing Wood and Hernhill-not common. P.

AGRIFOLIUM. R. S. 466.

ILEX Aquifolium. H. F. 505 .

The Holly Tree.

In Woods and Hedges-conmon. May. S.

AGRIMONIA. R. S. 202.

AGRIMONIA Eupatoria. H. F. 180.

Agrimony.

On the Sides of Fields and under Hedges - very common. June. S.

ALCEA vulgaris. R. S. 252 .

MALVA Alcea. H. F. 268.

Vervain Mallow.

In Paftures at Graveney and Hernhill - not common. Augult. P. 


\section{(3)}

ALLIUM fylveftre. R. S. 369 .

ALLIUM vineale. H. F. 121.

Crow-Garlick.

On the Wall that leads to Thorn Creek - pientifulin:

June. P.

ALLIUM fylveftre latifolium. R. S. 370 .

ALLIUM urfinum. H. F. 122.

Ramfons.

Amongt the Alders near Hernhill-Church - plentifully. May. P.

ALNUS. R. S. 442 .

BETULA Alnus. H. F. 506.

The Common Alder.

In moift Places-very common. July. S.

ALSINE hirfuta Myofotis. R. S. 349 .

CERASTIUM vilgatum. H. F. 175.

Narrow-leaved Moufe-ear Chickweed.

In Fields and Meadores-common. May. A.

ALSINE hirfuta Myofotis latifolia pracocior.

R. S. 348 .

CERASTIUM vifcosum. H. F. I75.

The broader leaved Moufe-ear Chickweed.

On the Walls of the Abbey-common. April. A. 


\section{$(4)$}

ALSINE major repens perennis. R. S. 347 .

CERASTIUM aquaticum. H, F. 177.

Great Marh Chickweed.

In Davington Brooks, and at Goodneftone - not common. July. P.

ALSINE marina foliis portulacæ. R.S. 35 I. ARENARIA peploides. H. F. 167.

Sea Chickweed.

On Shellnefs in Shepey-not common. July. P.

ALSINE minor multicaulis. R. S. 349 .

ARENARIA serpyllifolid. H. F. 167.

The lealt Chickweed.

On Roofs of old Houfes and on old Walls - common. May. A.

ALSINE fpergula dicta major. R. S. 351 .

SPERGULA arvenfis. H. F. 177 .

Corn Spurrey.

On fandy Grounds in Hern-Hill-common. July. A.

ALSINE fpergulæ facie media. R. S. $35 \mathrm{r}$. ARENARIA marina. H. F. 169.

Sea Spurrey.

On the Paths to Holly Shore-common. June: A. 


\section{( 5 )}

ALSINE foliis caryophylleis. R. S. 344: SAGINA erecza. H. F. 64 .

The leaft Stichwort.

In Nagden Marhes-not common. May. A.

ALSINELLA mufcofo flore repens. R. S. 345 . SAGINA procumbens. H. F. 63.

Pin Pearl-wort, Chickweed breakftone.

On old Walls about the Town-common. - June. A:

ALTHÆA vulgaris. R. S. 252.

ALTHA:A officinalis. H. F. 267.

Marh-Mallow.

In the Marßhes at Clapgate and Goodneftone-common. Augurt. P.

ANACAMPSEROS, vulgo Faba craffa. R.S. 269. SEDUM Telepbium. H. F. 170.

Orpine, or Live-long.

In Byfing and Cockfet Woods - not common. Auguif. $P$.

ANAGALLIS flore phœniceo. R. S. 282;

ANAGALLIS arvenfis. - H. F. 73.

Male Pimpernel.

In Fields and Gardens-common. May-Augurt. A,

ANAGALLIS Jutea. R. S. 282.

LYSIMACHIA nemorum. H, F. 73.

Yellow Pimpernel.

In Jud's Wood, Ofpringe=uncommon. June. P. 


\section{(6)}

ANBLATUM Cordi five Aphyllon. R. S.*288.

\section{LATHREA Squamaria. H.F. 233.}

Toothwort.

Under Hedges near Mr. Jenning's Houfe in Pluckley

-very uncommon. April. P.

ANEMONE nemorum. R. S. 259.

ANEMONE nemorosa. H. F. 208.

- Wood Anemony.

In Woods and Hedges-very common. April. P.

ANGELICA fylventris. R. S. 208.

ANGELICA fylveftris. H. F. I03.

Wild Angelica.

Near Stone-Bridge, and in Byfing Wood - not common. July. P.

ANGELICA fylveftris minor feu erratica.R.S.208. IEGOFODIUM Podagraria. H. F. III.

Herb Gerard, Gout-weed, or Afh-weed.

In Gardens and under Hedges, near Boughton Church -not common. May. P.

ANONIS maritima procumbens. R. S. $33^{2}$.

ONONIS repens. H. F. 273 .

Creeping Reft-harrow.

On the Sand Downs near Deal-uncommon. July. P. 


\section{$(7)$}

ANONIS fpinofa flore purpureo. R. S. 332 .

ONONIS spinofa. H. F. 273.

Reft-harrow, Cammock, Petty Whin.

In the Marghes at Clapgate-common. July. P.

ANONIS non fpinofa, R. S. 332 .

ONONIS arvenfis. H. F. 273.

Corn Reft-harrow, or Cammock.

In Corn Fields at Prefton and Ofpringe-common. July. P.

ANTIRRHINUM majus. R. S. omittitur.

ANTIRRIHINUM majus. H. F. 239.

The greater Snap-Dragon.

On old Walls about the Torn-common. June to September. P.

APARINE. R. S. 225.

GALIUM Aparine. H. F. 57.

Cleavers, or Goofe-grafs.

Under Hedges-very common. Auguft. A.

APARINE paluftris minor Parifienfis. R. S. 225.

GALIUM uliginofum. H. F. ${ }_{5}$.

Marih Goofe-grafs.

At the Boltom of Jud's Wood in Ofpringe - un: common. Auguft. P. 


\section{( 8 )}

APIUM paluftre et officinarum. R. S. 214.

APIUM graveolens. H. F. III.

Smallage.

'About the Sluice Bridge and the Keys-very common. Augurt. B.

AQUILEGIA fore fimplici. R. S. 273.

AQUILEGIA vulgaris. H.F. 207.

Common Columbines.

In Badgen Wood, and beyond Whitehill, Ofpringe -not common. June. P.

ARTEMISIA vulgaris. R. S. 190.

ARTEMISIA vulgaris. H.F. 312 .

Mugwort.

Under Hedges and on the Sides of Fields-very common. Auguft. P.

ARUM. R. S. 266.

ARUM maculatum. H. F. 342 .

Wake-Robin, Cuckow-Pint, Lords and Ladies. In Bady Places and under Hedges - very common. May. P.

ASPERULA. R. S. 224.

ASPERULA odorata. H. F. 55 .

- Woodroof.

In Ofpringe Woods-plentifully. May. P. 


$$
\text { (9) }
$$

ASPLENIUM. R. S. II8.

ASPLENIUM Ceteracb. H. F. 385 .

Spleen-wort, Milt-waft.

On Lenham CburcbWall - lately loft on the Walls of our Abbey. P.

ASTER maritimus cæruleus. R. S. 175 .

ASTER Tripolium. H. F. 3 rg.

Sea Star-wort.

In the Salt Marhes-plentifully. Auguft. P.

ASTER arvenfis cæruleus acris. R. S. $175^{\prime}$

ERIGERON acre. H. F. 314.

Blue flowered Flea-bane.

On oldWalls and on dry Banks-common. Augurt. P.

ASTER maritimus flavus, Crithmum chryfanthemum dictus. R.S. 174.

INULA cbritbmoides. H. F, 320.

Golden Sampire.

In the Markes of Shepey-uncommon. Augurt. P.

ASTRAGALUS luteus. R. S. 326 :

ASTRAGALUS glycyplyllos. H. F. $28 \mathrm{r}$.

Wild Liquorice, or Liquorice Vetch.

Under Hedges in Peafedown, near Whitehill, Ofpringe

-uncommon. July. P. 


\section{(10)}

ATRIPLEX anguftifolia maritima dentata. R.S. 152.

ATRIPLEX ferrata. H. F. 377 .

Narrow leaved Orache.

Near Sheernefs in Shepey-uncommon. Auguft. A.

ATRIPLEX anguftifimo et longiffimo folio. R. S. 153.

ATRIPLEX litoralis. H. F. 378 .

Grafs-leaved Orache.

Near Ramfgate Pier in Thanet uncommon. Auguft. A.

ATRIPLEX maritima. R. S. 152.

ATRIPLEX laciniata. H. F. 376 .

- Jagged Sea Orache.

Near Sheernefs in Shepey-uncommon. Auguit. A.

ATRIPLEX fylveftris anguftifolia. R.S. 15 I. ATRIPLEX patula. H.F. 377.

Narrow leaved wild Orache.

In Gardens and on Dungbills-common. Auguft. A.

ATRIPLEX fylveftris folio haftato feu deltoide. R. S. $15 \mathrm{I}$.

ATRIPLEX baftata. H.F. 377 .

Wild Orache with a fpear pointed Leaf.

In Gardens and wafte Places - common. September. A. 


\section{( II)}

ATRIPLEX maritima fcopariæ folio. R. S. ${ }_{53}$ :

ATRIPLEX litoralis. H. F. 378. $\beta$

Grafs-leaved Orache.

Near Margate Pier in Thanet - uncommon. Auguft. A.

ATRIPLEX maritima fruticofa. $R_{6} S .{ }_{53}$. A TRIPLEX portulacoides. H. F. 376 .

Sea Purflane.

Near Harty Ferry-plentifully. Auguft. P.

ATRIPLEX maritima perennis folio deltoide feu triangulari. R. S. 152 .

ATRIPLEX baftata. H, F. 377. $\beta$

Wild Sea Orache.

In the Brent Marßes-common. Augurt. A:

B.

BACCHARIS, monfpelienfium. R. S. 179

CONYZA fquarrosa. H. F. 314.

Plowman's Spikenard.

On Beacon Hill and dry Banks - not uncommon. Auguft. B.

BALLOTE. R. S. 244 .

BALLOTA nigra. H. F. 227.

Stinking Horehound.

Under Hedges-very common. Auguif, A.

BELLA. 


\section{(12)}

BELLADONNA. R.S. 265 .

ATROPA Belladonina. H.F. 79.

Deadly Night-hade, or Dwale.

Amongft the Bufhes under Prefton-Houfe Wallvery uncommon. June and July. $P$.

BELLIS fylveftris minor.' R. S. 184 .

BELLIS perennis. H. F. 320 .

Common Daify.

In Meadows - very common. From March to October. P.

BERBERIS dumetorum. R. S: 465 .

BERBERIS vulgaris. H. F.

The Berberry-bufh, or Pipperidge-burh.

In a Hedge beyond Queen Court, Ofpringe - uncommon. May. S.

BETONICA. R. S. 238.

BETONICA Officinalis. H. F. 220.

Wood Betony.

In Woods and under Hedges-common. July. P.

BETA fylveftris maritima. R. S. 38 .

BETA vulgaris. H. F. 93.

Sea Beet.

On the Sea Walls at Ham ard Graveney-uncommon. Auguft. B. 


\section{( 13$)$}

BETULA. R. S. 443.

BETULA alba. H. F. 354 .

The Birch Tree.

In Woods-common. April. S.

BIFOLIUM majus, feu Ophrys major quibufdam. R. S. 385 .

OPHRYS ovata. H.F. 338 .

Common Twayblade.

In Cockfet and Jud's Woods-common. June. P.

BLITUM Atriplex fylveftris dictum. R. S. I 54. CHENOPODIUM album. H.F. $9 \mathrm{r}$.

Common wild Orache.

In uncultivated Places, and on Rubbif — common. Augurt. A.

BLITUM foetidum Vulvaria dicum. R.S. 156 . CHENOPODIUM Vulvaria. H. F. 92 .

Stinking Orache.

Under Walls about the Torn-uncommon. Augut. A.

BLITUM Ficus folio. R. S. 155.

CHENOPODIUM ferotinum. H. F. 9 r.

Late flowering or Fig-leaved Blite.

In Gardens and on Rubbihs-common. A. 


\section{( 14$)$}

BLITUM Kali minus album dietum. R.S. 156 . CHENOPODIUM maritimum. H. F. 92 .

Sea Blite, or white Glafs-wort.

In the Salt Marßies-common. Auguit. A.

BLITUM perenne Bonus Henricus dictum. R.S. 156 .

CHENOPODIUM Bonus Henricus. H.F. 89:

Common Englith Mercury, or All-good.

By Way-sides in Ofpringe-not uncommon. July. P.

BLITUM Pes anferinus dictum. R. S. 154.

CHENOPODIUM murale. H. F. 90.

Common Goofe-foot, or Sow-bane.

Iis Gardens and on Rubbijh not uncommon. Auguif. A.

BLITUM Pes anferinus dictum acutiore folio.

R.S. 154.

CHENOPODIUM rubrum. H. F. 90 .

Sharp-leaved Goofe-foor, or Sow-bane.

On Dungbills and Rubbißs-common. Augutt. A.

BLITUM rubrum minus. R. S. 157.

AMARANTUS Blitum. H. F. $35^{6}$.

The leaft Blite.

On Rubbish and in Gardens-common. Auguft. A. 
BORRAGO hortenfis. R. S. 228 .

BORRAGO ifficinalis. H. F. 68.

Borrage.

On old Walls, and by Rocd-fides near the Torennot common. June. $P$.

BORRAGO fempervirens. R. S. $22 \%$

ANCHUSA fempervirens. H. F. 66.

Evergreen Alkanet.

On Rubbifs near Prefton Church Yard - very uncommon. June. P.

BRASSICA maritima arborea five procerior ramofa. R. S. 293.

BRASSICA oleracea. H. F 253.

Sea Cabbage.

On the Cliffs between Deal and Dover - plentifully. April. B.

BRYONIA alba. R. S. 26 .

BRYONIA alba. H. F. 373 .

White Briony.

In Hedges - common. May. P.

BUGLOSSA fylveftris minor. R. S. 22\%:

LYCOPSIS arvenfis. H. F. 69.

Small wild Buglofs.

In Corn-fields near Prefton Church - uncommon. May. A. BUGLOS: 


\section{(16)}

BUGLOSSUM arvenfe annuum. Lithofpermi folio. R. S. 227.

LITHOSPERMUM arvense. H. F. 66.

Baftard Alkanet.

In Corn-fields in Prefton and Hernhill

July. A.

BUGULA. R. S. 245.

AJUGA reptans. H. F. 219 :

Bugle.

In Byfing Wood - common. May. P.

BULBOCASTANUM. R. S. 209.

BUNIUM Bulbocaftanum. H. F. 105.

Earth-nut, or Hog-nut.

In'Byfing Wood and Hernhill-common. June. P.

BUPLEURUM minimum. R. S. $22 \mathrm{r}$.

BUPLEURUM tenuifimum. H. F 97.

The leaft Hare's-ear.

Near Sheernefs in Shepey-uncommon. Auguft. A.

BUPLEURUM perfoliatum rotundifolium annuum. R.S. $22 \mathrm{I}$.

BUPLEURUM rotundifolium. H. F. 97 .

Thorow-wax.

In Corn-fields near Brogdale in Ofpringe-uncommon. July. A.

BUTO: 


\section{(I7)}

BURSA Paftoris. R. S. 306.

THLASPI Burfa Paftoris. H. F. 247\%

Shepherd's Purfe.

In Hop Grounds, and by Road-fides - very common: May to September. A.

BUTOMUS. R. S. 273.

BUTOMUS umbellatus. H. F. 152:

The flowering Ruh, or Water Gladiole.

In Dykes near Pluckley-uncommon. June. $\mathbf{P}$.

*-BUXUS. R. S. 445 .

BUXUS fempervirens. H. F. $355^{\circ}$

The Box Tree.

A few large Trees near Houjes in Sheldwich - very uncommon. $S$.

\section{C.}

CAKILE quibufdam, aliis Eruca marina et Rha: phanus marinus. R.S. 307 .

BUNIAS Cakile.

Sea Rocket.

Near Sheernefs in Shepey, and Cliff's End irs Thanet - uncommon. June. A.

CALAMINTHA humilior folio rotundiore.

R. S. 243 .

GLECHOMA bederacea. H.F. 244.

Ground Ivy. Alehoof.

In Bady Places, and under Hedges - very common. May. P: 


\section{(18)}

CALAMINTHA vulgaris. R. S. 243.

MELISSA Calamintba. H. F. 230.

Common Calamint.

By the Sides of Roads, and under Hedges - common. Augutt. P.

CAMPANULA rotundifolia. R. S. $27 \%$.

CAMPANULA rotundifolia. H. F. 80.

The leffer round leaved Bell Flower.

By Way-sides in Ofpringe and Boughton-not common. Auguft. P.

CAMPANULA vulgatior foliis urticæ. R.S. 276 .

CAMPANULA Trachelium. H. F. $8 \mathbf{I}$.

Canterbury Bells, or Great Throat-wort.

In Woods and Hedges - very common. - With a wbite Flower in Hernhill - very uncommon. July. P.

CAPRIFOLIUM germanicum. R. S. 458 .

LONICERA Periclymenum. H. F. 80.

Common Honey-fuckle, or Woodbine.

In Woods and Hedges - very common. April to Auguft. S.

CARDAMINE. R. S. 299:

CARDAMINE pratenfis. H. F. 256.

Common Ladies Smock, or Cuckow-flower. In moift Meadows-very common.' A pril. P. 


\section{(19)}

CARDAMINE parviflora. H. F. 257.

Small foowered Ladies Smock.

In Meadores near Pluckley-uncommon. A pril. A.

CARDIACA. R.S. 239.

LEONURUS Cardiaca. H. F. 228.

Mother-wort.

Near Cockfet, in Ofpringe — very uncommon. July. B.

CARDUUS acaulos minore purpureo flore.

R. S. 195.

CARDUUS acaulos. H. F. 308 .

Dwarf Carline Thiftle.

Upon Beacon Hill-not unconsmon. July. P.

CARDUUS caule crifpo. R. S. 194.

CARDUUS crippus. H. F. 306 .

Thiftle upon Thifte.

By the Road-fides, and on dry Banks in Ofpringe common. June. A. .

CARDUUS lanceolatus. R. S. 195.

CARDUUS lanceolatus. H. F. 305 .

Spear-Thiftle.

In uncultivated Ground, and under Hedges - common. July. B.

CAR: 


\section{(20)}

CARDUUS Mariæ. R. S. 195.

CARDUUS marianus. H. F. 307.

Milk Thiftle, or Lady's 'Thiftle.

By the Way-fide at Davington, and by the Sea Walls beyond Nagden-riot uncommon. July. A.

CARDUUS Mariæ hirfutus non maculatus.

R. S. 194.

CARDUUS marianus. H. F. $307 . \beta$.

Ladies Thiftle without Spots.

In Graveney Marßhes - uncommon. July. A.

CARDUUS nutans. R. S. 193.

CARDUUS nutans. H. F. 306 .

Murk Thiftle.

On Badgen Downs - plentifully. July. B.

CARDUUS paluftris. R. S. 194.

CARDUUS paluffris. H.F. 306 .

Marfh Thinle.

In the Marßhes - common. July. B.

CARDUUS fpinofifrmus capitulis minoribus.

R. S. . 194.

CARDUUS acanthoides. H. F. 306.

Welted Thiftle with fmall Flowers.

Upon Graveney Marßs Sea Wall-common. July. A. 


\section{(2I)}

CARDUUS ftellatus. R. S. 176 .

CENTAUREA Calcitrapa. H. F. 326 .

Star Thiftle.

In Graveney Marhes - common. July. A.

CARDUUS tomentofus Acanthium dictus vulgaris. R. S. 196 .

ONOPORDUM Acantbium. H. F. 308.

Cotton Thiftle.

In uncultivated Places, and by the Road-side-common. July. B.

CARDUUS vulgatifimus viarum. R.S. 194: SERRATULA arvenfis. H. F. 305.

Common Way-Thiftle, or Creeping Thiftle. In Fields and Byways - very common. July. P.

CARLINA fylveftris quibufdam, aliis Atractilis. R. S. 175 .

CARLINA vulgaris. H. F. 309 .

The common wild Carline Thiftle. In dry Fields and Paftures - common. June. B.

CARYOPHYL.LATA. R. S. 253.

GEUM urbanum. H. F. 198.

Herb Bennet, or Common Avens. In Woods, and under Hedges-common. July. P. 
CARYOPHYLLATA montana purpurea. R.S.

253.

GEUM rivale. H. F. 198.

Water Avens.

In a Wood near Barber's Mill in Hothfield - very uncommon. July. P.

CARYOPHYLLUS holofteus arvenfis glaber flore majore. R. S. 346.

STELLARIA Holofea. H. F. 166.

The greater Stich-wort.

Under Hedges, and on the Sides of Fields — very common. April. P.

CARYOPHYLLUS holofteus arvenfis glaber flore minore. R. S. 346 .

STELLARIA graminea. H. F. 166.

The leffer Stich-wort.

Under Hedges, cbiefly on a dry Soil-common. July. P. CARYOPHYLLUS holofteus arvenfis medius. R.S. 347 .

STELLARIA graminea. H. F. 166 . $\beta$.

The middle Suich-wort.

Upon Beacon Hill - not uncommon. July. P.

CARYOPHYLLUS latifolius barbatus minor annuus flore minore. R. S. 337 .

DIANTHUS Armeria. H. F. 161.

Deptford Pink.

On Beacon Hill, and dry Paffures in Ofpringe - not uncommon. July. A.

CAS. 


\section{(23)}

CASTANEA. R. S. $44^{\circ}$.

FAGUS Caftanea. H. F. 359 .

The Chefnut Tree.

In Byfing and otber Woods-not uncommon. May. S.

CATANANCE leguminofa quorundam. R. S.

325 .

LATHYRUS Niffolia. H. F. 275 .

Crimfon Grafs-Vetch.

On Beacon Hill - not uncommon. June. A:

CAUCALIS minor flofculis rubentibus. R.S. 219.

CAUCALIS Antbrifcus. H. F. 99.

Hedge Parney.

In buby Grounds, and on Ham Wall-not uncommon: July. B.

CAUCALIS fegetum minor, Anthrifco hifpido fimilis. R. S. 220.

CAUCALIS arvenfis. H.F. 98.

Small Corn Parfley.

In Fields, and amongft Corn-common. Auguft. A.

CENTAURIUM luteum perfoliatum. R. S. 287. BLACKSTONIA perfoliata. H.F. 146 .

Yellow Centory.

In dry chalky Fields - common. July. A.

CEN- 


\section{( $\left.24^{*}\right)$}

CENTAURIUM minus. R. S. 286. GENTIANA Centaurium. H. F. 88.

Leffer Centory.

In áry Pafures-very common. July to Oetober. A.

With a white Flower, near Minfter in Shepey but uncommon.

CERASUS fylveftris fructu rubro. R.S. $46_{3}$. PRUNUS Avium. H. F. 187.

Commion wild Cherry.

In Woods - common. April. S.

CERASTIUM hirfutum minus parvo flore.

R. S. 348 .

CERASTIUM Jemi-decandrium. H. F. 175 . The leaft Moufe-ear Chickweed.

On Walls and dry Soils - common. April. A.

CEREFOLIUM fylveftre. R. S. 207.

CHÆROPHYLLUM temulum. H.F. 108. Wild Chervil.

Under Hedges - very common. July. A.

CHAM EMELUM fotidum. R. S. 185 .

ANTHEMIS Cotula. H. F. 223.

Stinking Mayweed.

In Corn Fields - too common. June. A. 


\section{(25)}

CHAM AEMELUM inodorum. R. S. 185. MATRICARIA inodora. H.F. 322.

Field Feverfew.

On the fides of patbs in Corn-fields _ common. Auguit. A.

CHAMEMELUM odoratifimum repens fore fimplici. R. S. 185.

ANTHEMIS nobilis. H. F. 323 .

Sweet-fcented Camomile.

On Charing Heath-uncommon. Augurt. P.

CHAMÆMELUM vulgare. R.S, I 84. MATRICARIA Chamomilla. H.F. 322.

Corn Feverfew.

In cultivated Fields amongft Corn-common. June. A.

CHELIDONIUM minus. R. S. 246 .

FICARIA verna. H. F. 214.

Pilewort, or leffer Celandine.

In Meadows - very common. April. P.

CHENOPODIUM Betæ folio. R. S. 157.

CHENOPODIUM polypermum. H. F. 92.

Upright round leaved Blite, or Allfeed.

In the Conduit Row-not unccmmon. Augurt. A. 


\section{( 26$)$}

CHENOPODIUM erectum foliis triangularibus dentatis, fpicis e foliorum alis plurimis longis erectis tenuibus. R. S. 155 .

CHENOPODIUM urbicum. H. F. 89.

Upright Blite.

In the Conduit Row, and in Ditches-common. September. A.

CHENOPODIUM Stramonii folio. R. S. 154 . CHENOPODIUM bybridum. H. F. 90.

Maple-leaved Blite.

On Dungbills-not common. Auguft. A.

CHRYSANTHEMUM fegetum. R. S. 182.

CHRYSANTHEMUM Jegetum. H. F. 321 。

Corn Marigold.

In Corn-fields_- common. July. A.

CICHOREUM fylveftre. R. S. 172.

CICHOREUM Intybus. H. F. 303 .

Wild Succory.

In uncultivated Places, and by Sides of Fields - very common. Auguft. P.

CICUTA. R. S. 2 I5. CONIUM maculatum. H. F. 100.

Hemlock.

In Bady Places near the Town-common. July. A. 


\section{( 27 )}

CICUTARIA tenuifolia. R. S. 215 . ÆTHUSA Cynapium. H. F. 107.

The leffer Hemlock, or Fools Parley.

In Gatefield, and in Gardens - not uncommon. September. A.

CICUTARIA vulgaris. R. S. 207.

CHÆROPHYLLUM Jylveftre. H. F. 108.

Wild Cicely, or Cow-weed.

Under Hedges, and in Fields - common. June. A.

CIRCÆEA lutetiana. R. S. 289.

CIRC㤅 lutetiana. H. F. 9 .

Enchanters Night-hhade.

In Byfing Wood - common. July. P.

CLEMATIS daphnoides major. R. S. 268.

VINCA major. H. F. 77.

The greater Periwinkle.

In Hedges, near Houses at Ore and Sindal Farm uncommon. A pril. P.

CLEMATIS latifolia feu Atragene quibufdam: R. S. $25^{8}$.

CLEMATIS Vitalba. H. F. 217.

Great Wild Climber, or Traveller's Joy. In Hedges - very common. July. P. 


\section{( 28$)$}

CLINOPODIUM Origano fimile. R. S. 239.

CLINOPODIUM vulgare. H. F. 228.

Great wild Bafil.

In Hedges - very common. July. P.

COCHLEARIA folio finuato. R. S. 303 .

COCHLEARIA anglica. H. F. 248.

Sea Scurvy-grafs.

On the Sea Walls near Thorn-common. May. B.

CONVOLVULUS major. R.S. 275.

CONVOLVULUS Sepium. H. F. 74 .

Great Bindweed.

Under Hedges-very common. With a reddift purple

Flower, in the Brent Hedge; but very uncommon. Auguft. P.

CONVOLVULUS maritimus Soldanella dietus.

R.S. 276 .

CONVOLVULUS Soldaneila. H. F. 75 .

Sea Bindweed.

On the Sand Downs near Sandwich - not common. July. P.

CONVULVULUS minor vulgaris. R.S. 275 . CONVOLVULUS arvenfis. H. F. 74 .

Small Bindweed.

In Fields, and by Road-fides-very common. July. P. 


\section{(29)}

CONYZA media. R. S. 174 .

INUI_A dy fenterica. H. F. 320.

Middle Fleabane.

About the Abbey Pond - common. Auguft. P.

CONYZA minor. R. S. 174 .

INULA Pulicaria. H. F. 320 .

Small Fleabane.

About the Conduit-row, and at Downe's-forftal in Hernhill - not uncommon. September. A.

CORNUS fæmina. R. S. 460 .

CORNUS sanguinea. H. F: $5^{\text {s. }}$

The female Cornel, Dog-berry Tree, or Prick-wood.

In Hedges - commen. June. S.

CORYLUS fylveftris. R. S. 439 .

CORYLUS Avellane. H. F. 360 .

The Hazle-nut Tree:

In Woods and Hedges-very common. March. S.

COTYLEDON vera radice tuberofa. R. S. $27 \mathrm{I}$. COTYLEDON Umbilicus Veneris. H. F, 169. Navel-wort, Kidney-wort, or Wall Penny-wort. On Tenterden Cburch, and in a Stone Pit at Boughton Monchelfea-very uncommon. June. P.

$\mathrm{D}_{3}$ CRACCA. 


\section{( 30$)$}

CRACCA. R. S. 322.

VICIA Cracca. H. F. $27 \%$.

Tufted Vetch.

In the Hedges of the Beacon Farm - uncommon.

July. A.

CRACCA minor. R. S. 322.

ERVUM birfutum. H. F. 280.

Sinall wild Tare, Tine-Tare, or hairy Tare.

In Rickefy-lane Hedge, Selling - not uncommon.

july. A.

CRACCA minor filiquis fingularibus flofculis carulefcentibus. R. S. 322 .

ERVUM tetraspermum. H. F.

Tine-Tare with fmooth Pods; or fmooth Tare. In Fields and under Hedges-common. June. A.

CRITHMUM marinum. R.S. 217 :

CRITHMUM maritimum. H. F. IOI.

\section{Sampire.}

On the Cliff's betroeen St. Margaret's and Dover plentifully. Auguft. P.

CRUCIATA. R. S. 223.

VALANTIA Cruciata. H. F. 375 .

Crofs-wort, or Mug-weed.

In Hedges in Preston and Ofpringe-not uncommon.

June. F. 


\section{( 31 )}

CUSCUTA major. R. S. $28 \mathrm{I}$.

CUSCUTA europea. H. F. 89 .

Dodder, Hell-weed, Devil's-guts.

Upon leguminous Plants - common. July. A

CYANUS. R. S. 198.

CENT AUREA Cyamus. H. F. 325 .

Blue Bottles, or Corn Bottles.

In Corn-fields - very common. July. A.

CYNOGLOSSUM. R.S. 226 .

CYNOGLOSSUM officinale. H. F. 6\%. . . .

Great Hound's Tongue.

By the Road Sides near Boughton Chalk Pits - not: common. June. P.

CYNOGLOSSUM folio virenti. R. S. 226 . CYNOGLOSSUM officinale H, F. 67. $\beta_{\text {e }}$

The leffer green leaved Hound's Tongue. Near Sandwich - not common. June. P.

\section{D.}

DAMASONIUM ftellatum Dalechampii.

R.S. 272 .

ALISMA Damafonium. H. F. 138 .

Star-headed Water Plantain.

In a Field-pond of Mr. Jacob's Farm, at Ealt Church' in Shepey - very uncommon. June to Augurt. $P$. DAU: 


\section{(32)}

DAUCUS vulgaris. R. S. 218 .

DAUCUS Carota. H. F. 99.

Wild Carrot, or Bird's Neft.

In Paftures, and by Way-fides-very common. July. B.

* DELPHINIUM fegetum flore caruleo.

R. S. 273 .

DELPHINIUM Consolida. H. F. 207.

Wild Larkfpur.

In King's Field, amongft Corn - very uncormmon.

June. A.

DENS-LEONIS. R. S. 170.

LEONTODON Taraxacum. H. F. 297.

Dandelion.

In Meadowes and Paftures-very common. May.. P.

DENS-LEONIS hirfutus, Hieracium dietus.

R. S. 127.

LEONTODON bifpidum. H. F.. 297.

Rough Dandelion, or Dandelion: Hawk-weed.

In Meadores and Paftures - common. June. P.

DIGITALIS purpurea. R. S. * 283 .

DIGIT ALIS purpurea. H. F. 240.

Purple Fox Gluve.

In.Woods and Hedges - common: Ju'y. B. 


\section{( 33 )}

DIPSACUS minor feu Virga paftoris. R. S. 192.

DIPSACUS pilófus. H. F. 49 .

Small wild Teafel, or Shepherd's Rod.

In the Road Hedge leading from Plomford to Badgen

Downs - uncommon. July or Auguft. B.

DIPSACUS fylveftris feu Labrum Veneris.

R.S. 192.

DIPSACUS fylveftris. H.F. 49 .

Wild Teafel.

In uncultivated Places, and under Hedges - very

common. July. B.

\section{E.}

ECHIUM alterum five Lycopfis anglica. R.S. 228. ECHIUM anglicum. H. F. 70.

Englin Viper's Buglofs.

By Way fides near Whitehill in Ofpringe - not uncommon. Auguft. $P$.

ECHIUM vulgare. R. S. 218.

ECHIUM vulgare. H. F. 69 .

Viper's Buglors.

On barren Ground, and upon old Walls - very common. July. P.

EQUISETUM arvenfe longioribus fetis. R. S. 130.

E.QUISETUM arvense. H. F. $3^{80}$.

Corn Horfe-tail.

In moift Fields amongft Corn-common. March. P. 


\section{( $34-)$}

EQUISETUM majus. R. S. 130.

EQUISETUM fluviatile. H.F. 381 .

River Horfe-tail.

In the River about the Powder Mills — common. May. P.

EQUISETUM paluftre. R. S. I 3 T.

EQUISETUM paluftre. H. F. 380.

The leffer Marnh Horfe-tail.

In the Corn Fields near Nagden — not uncommon. June. $P$.

EQUISETUM fylvaticum. R. S. 130. EQUISETUM fylvaticum. H. F. 380.

Wood Horfe-tail.

In the fwampy Ground near Ore Mill - not common. May. P.

ERICA Brabantica folio Coridis hirfuto quaterno. R. S. 471 .

ERICA tetralix. H.F. 144 .

Crofs-leaved Heath.

At the Bottom of Jud's Wood - not common. Au$\therefore$ guft. $\mathrm{S}$.

ERICA tenuifolia. R. S. 471 .

- ERICA cinerea. H. F. I44.

Fine leaved Heath. -

Upon Charing Heath - not common. Auguft. S.

ERICA 


\section{( 35 )}

ERICA vulgaris. R. S. 470 .

ERICA vulgaris. H. F. 144.

Common Heath, or Ling.

In Woods-very common. June to September. P.

ERUCA aquatica. R. S. 297.

SISYMBRIUM fylveftre. H. F. 258.

Water Rocket.

In Ham Ponds near Sandwich-uncommon. June. P.

ERUCA hirfuta filiqua cauli appreffa. Eryfimum vulgare. R. S. 298.

ERYSIMUM officinale. H. F. 250.

Hedge Muftard.

Under Hedges, and by Road Sides - very common.

May. A.

ERUCA lutea feu Barbarea. R. S. 297.

ERYSIMUM Barbarea. H. F. 251.

Winter Crefs or Rocket.

Near the Powder Mills_not uncommon. May. P.

ERYNGIUM marinum. R. S. 222.

ERYNGIUM maritimum. H. F. 95.

Eryngo, or Sea Holly.

On the Sbore between Graveney and Sea Salter uncommon. July. P. 


\section{$(36)$}

ERYSIMUM Sophia dictum. R. S. 298.

SYSIMBRIUM Sopbia. H. F. 259.

Flix-weed.

By the Road Sides near Sittingbourn - uncommon.

June. A.

EUONYMUS vulgaris. R. S. 468.

EUONYMUS europaus. H. F. 84.

The Spindle Tree, or Prick-wood.

In. Woods and Hedges - common. May. S.

EUPATORIUM Cannabinum. R. S. 179.

EUPATORIUM cannabium. H. F. 310.

Conmon Hemp Agrimony, or Dutch Agrimony. About the Abbey Pond-common. Augurt. P.

EUPHRASIA. R. S: *284.

EUPHRASIA officinalis. H. F. 234 .

Eye-bright.

In moft upland Paftures-very common. A ugurt. A.

EUPHRASIA pratenfis rubra. R. S. * 284.

EUPHRASIA Odontites. H. F. 234 .

Red Eye-bright.

In the Ways and Corn Fields about Prefton- very cossmon. Auguft. A. 


\section{$(37)$}

F.

FAGUS. R. S. 439.

FAGUS fylvaticus. H. F. 360.

The Beech Tree.

In Woods - common. May. S.

* FEGOPYRUM. R. S. I44. POLYGONUM Fagopyrum. H.F. 150. Buck-wheat, or Brank.

In Corn fields near Beacon Hill-uncommon. July. A.

FEGOPYRUM fcandens fylveftre. R. S. 144 . POLYGONUM Convolvulus. H.F. 149.

Black Bind.weed.

In Corn ficlds and Hedges - not uncommon. June to September. A.

FERRUM equinum germanicum filiquis in fummitate. R. S. 326 .

HIPPOCREPIS comosa. H. F. $28 \mathrm{I}$.

Tufted Horfe-fhoe Vetch.

On cbalky Ground in Ofpringe-common. July. A.

FILIPENDULA. R. S. 259.

SPIR E A Filipendula. H. F. 190.

Common Dropwort.

Upon Beacon Hill - not uncommon. July. P.

E

FILIX 


\section{$\left(3^{8}\right)$}

FII IX Fœmina. R. S. 124.

PTERIS aquilina. H. F. 384 .

Female Fern, or Common Brakes.

In Woods - very common. Augurt. P.

FILIX mas vulgaris. R. S. 120.

POLYPODIUM Filix mas. H. F. 389 .

Common Male Fern.

On hady Bcnks under Hedges - common. $\mathbf{P}$.

FILICULA faxatilis ramofa maritima noftras.

$\because$ R. S. 125.

PTERIS aquilina. H. F. $384 . \quad \beta$.

Small-branched Stone-Fern.

On Walls about the Town-uncommon. Auguft. P.

FCENICULUM vulgare. R. S. 217.

ANETHUM Freniculum. H. F. Iro.

Common Fennel, or Finkle.

By the Road-fide as you enter the Town - not uncom-. . mon. Auguft. P.

FRAGARIA. R. S. 254.

FRAGARIA vefca. . H. F. 194.

Strawberry.

In W.oods - very comimon. A pril. P. 


\section{( 39$)$}

FRAGARIA fterilis. R. S. 254.

FRAGAKIA ferilis. H. F. 195.

Barren Strawberry.

On barren Lanás - very common. A pril. P.

FRANGULA five Alnus nigra baccifera. $R$.

465.

RHAMNUS Frangula. H. F. 83.

The black Berry-bearing Alder.

In Badgen Wood - not common. May. S.

FRAXINUS. R. S. 469 .

FRAXINUS excelfior. H. F. 379 .

The common Afh Tree.

In Wocds and Hedges - common. A pril. S.

FUMARIA vulgaris. R. S. 204.

FUMARIA officinalis. H. F. 270.

Fumitory.

In Fields and Gardens._common. May. A.

FUMARIA alba latifolia. R. S. 204. FUMARIA clariculata. H. F. $27^{\circ}$.

Climbing Fumitory.

In moift Hedges in Davington and Ofp:inge - not common. Augurt. A. 
G.

GALE frutex odoratus feptentrionalium. R.S. 443. MYRICA Gale. H. F. 368.

Sweet Willow, Goule, Dutch Myrtle.

On Willfborough Lees, near Anford - plentifully. May. S.

GALEOPSIS legitima Diofcoridis. R. S. 237. STACHYS fylvatica. H. F. 227.

Hedge Nettle.

fn Woods and Hedges-very common. Augurt. A.

GALLIUM luteum. R. S. 224.

GALLIUM verum. H. F. 55 .

Yellow Ladies Bedftraw, or Cheefe-renning. On the Sides of Fields-very common. July. P.

GENISTA angulofa trifolia. R. S. 474. SPARTIUM fcoparium. H. F. 271.

Common Broom.

In Woods and dry Fields - very common. June. P. GENISTA minor afpalathoides. R. S. 475 . GENISTA anglica. H. F. 272.

Needle Furze, or Petty Whin.

Amongft Furze which grows on wet Land - not common. May. P. 


\section{(4I)}

GENISTA fpinofa vulgaris. R. S. 475 .

ULEX europaus. H. F. 272.

Furze, Whins, or Gorfe.

On Beacon Hill-very common. March to June. P.

GENISTELLA tinetoria. R. S. 474.

GENISTA tinctoric. H. F. 272.

Dyers-weed, Green-wood.

In the Marhes near Holly Shore - not common. July. P.

GENTIANELLA fugax autumnalis. R.S. 275 . GENTIANA Amarella. H. F. 87.

Autumnal Gentian.

In dry Paftures and Ofpringe Chalk Pits - not uncommon. Auguft. A.

GERANIUM Cicutæ folio inodorum. R. S. 357 . GERANIUM cicutarium. H. F. 262.

Field Crane's-bill without Scent, or Hemlockleaved Crane's-bill.

By Road sides, and on old Weils - not uncommon. April to June. A.

GERANIUM columbinum. R. S. 359 .

GERANIUM molle. H. F. 265 .

Common Dove's-foot Crane's bill.

Uider Hedges - very common. June. A.

$$
\text { F. } 3
$$




\section{(42)}

GERANIUM columbinum humile flore cæruleo.

R. S. 359 .

GERANIUM pufillum. H. F. $266^{\circ}$.

Small flowered Duve's-foot Crane's-bill.

By $W$ ayjides leading to Thorn-common. June. A.

GERANIUM columbinum majus foliis diffectis.

R. S. 359 .

GERANIUM diffectum. H. F. 266.

Jagged leaved Dove's-foot Crane's-bill.

In dry Fields in Ofpringe - not uncommon. May to July. A. .

GERANIUM columbinum diffectis foliis pediculis forum longifimis. R. S. 359.

GERANIUM columbinum. H. F. 266.

Long-ftalked Dove's-foot Crane's-bill.

Upon Oldwives Lees - not common. July. A.

GERANIUM robertianum. R. S. $35^{8}$.

GERANIUM robertianum. H. F. 264.

Herb Robert.

In Hedger, and on Rubbiß-very common. June. B.

GERANIUM pufillum fupinum maritimum.

R. S. 357 .

CERANIUM maritimum. H, F. $26_{3}$.

Sea Crane's-bill.

On the Sand Dowens near Deal - not common. Auguit. $P$.

GLAUX 


\section{( 43 )}

GLAUX maritima. R. S. 285 .

GLAUX maritima, H. F. 86.

Sea Milkwort, or black Saltwort.

In the Brent Marßhes - common. July. P.

GNAPHALIUM longifolium humile ramofuns capitulis nigris. R.S. 181 .

GNAPHALIUM uliginofum. H. F. 313 .

Black-headed, long leaved, low branched cudweed.

In moift Places in Hernhill - not uncommon. A utguit. A.

GNAPHALIUM anglicum. R.S. I80.

GNAPHALIUM fylvaticum, H. F. 3'3.

Upright Cudweed.

In Judd's Wood-common. Auguft. B.

GNAPHALIUM minimum. R.S. 181 .

FILAGO montana. H. F. 328.

The leatt Cudweed.

In dry Sandy Meadows, and among Cors _ common. July. A.

GNAPHALIUM minus feu Herba impia.

R.S. 180.

FILAGO germanica. H. F. 323 .

Common Cudweed.

In dry. Meadows ond by Road fides-common. July. A. GROS- 


\section{$(44)$}

* GROSSULARIA. Miller Botan Officinal. 220. RIBES Uva crispa. Lin. Syftem. Nat. Tom. $1 I$. p. 184.

The Goofeberry Burh.

In Several Hedges beyond White Hill, Ofpringe. April. S.

H.

HEDERA communis major et minor. R. S. 459 . HEDER A Helix. H. F. 85.

Climbing, or berried Ivy, alfo barren and creeping Ivy.

In Woods and Hedges - very common. OEtober. S.

HELIANTHEMUM vulgare. R. S. 34I.

CISTUS Heliantbemum. H. F. 205.

Dwarf Ciftus, or little Sunflower.

On dry cbalky Banks - very common. June. P.

HELENIUM. R. S. 176 .

INULA Helenium. H. F. 319.

Elicampane.

On the moiff Ground near the Half-way Houfe to Canterbury - very uncommon. A uguift. P.

HELLEBORUS niger hortenfis. R. S. 271 . HELLEBORUS viridis. H.F. 215.

Wild black Hellebore.

On the Cliff beyond Weftfeld in Pluckley - uncommon. April. P. 


\section{(45)}

HELLEBORASTER maximus. R.S. $27 \mathrm{r}$.

HELLEBORUS fatidus. H. F. 215.

Great baftard black Hellebore, Bear's-foot, or

Setter-wort.

By the Road fide, up the Chalk-hill, about a Mile Nortb-reft from Charing-uncommon. March. P.

HELLEBORINE altera atro-rubente flore.

R. S. $3^{8} 3$.

SERAPIAS latifolia. H. F. $34 \mathrm{I}$. $\beta$.

Broad-leaved baftard Hellebore, a Variety.

In Orpringe Woodis - uncommon. Auguit. P.

HELL,EBORINE latifolia montana. R. S. $3^{8} 3$.

SERAPIAS latifolia. H. F. 341 .

Broad leaved baftard Hellebore.

In King's Wood - uncommon. July. P.

HELLEBORINE latifolia flore albo claufo.

R. S. $3^{84}$.

SERAPIAS longifolia. H. F. 341.

White flowered baftard Hellebore.

In Badgen and Jud's Woods-uncommon. June. P.

HERBA Paris. R. S. 264.

PARIS quadrifolia. H. F. 150.

Herb Paris, True-love, or One-berry.

In Woods near Doddington-not common. May. P.

HES. 


\section{(46.)}

HESPERIS allium redolens. R. S. 293.

ERYSIMUM Alliaria. H. F. $25^{1}$.

Jack by the Hedge, or Sauce alone.

Under Hedges - very common. May. P.

HIER ACIUM Echioides capitulis Cardui benedicti. R. S. 166.

PICRIS Ecbioides. H. F. 294.

Ox's Tongue, or Lang de Boeuf.

In Hedges and Paftures going to Thorn Creek

common. July. A.

HIERACIUM fruticofum anguftifolium majus.

R. S. 168.

HIERACIUM umbellatum. H. F. 300 .

Narrow leaved bufhy Hawk-weed.

In Ofpringe Ckalk Pits, and by Way-fides at Dunkirk

- uncommon. Augutt. P.

HIERACIUM fruticofum latifolium hirfutum. R. S. $18 \%$.

HIERACIUM Sabaudum. H. F. 300 .

- Bufhy Hawk-weed with broad rough Leaves.

In Hedges about Sheldwich - not common. July. P.

HIERACIUM longius radicatum. R. S. $16_{5}$. HYPOCHÆRIS radicata. H. F. 302.

- Long-rooted Hawk-weed.

On Beacon Hill - common. June. P. 


\section{(47)}

HIERACIUM luteum glabrum five minus hirfutum. R. S. 165.

CREPIS tectorum. H. F. $30 \mathrm{r}$.

Smooth Succory Hawk-weed.

In Fields and on old Walls - common. June to Sep. tember. A.

HIER ACIUM maximum Chondrillæ folio afperum. R. S. 166.

CREPIS biennis. H. F. 301 .

Rough Succory Hawk-weed.

In. Hedges near Sittingbourn-uncommon. Auguft. B.

HIERACIUM minus præmorfa radice. R.S. 164 .

LEONTODON autumnale. H. F. 297.

Hawk-weed with bitten Roots, or yellow Devil's Bit.

In Corn fields in Hernhill-common. Auguft. P.

HORMINUM fylveftre Lavendulæ fore. R. S. 237.

SALVIA verbenaca. H. F. 9.

Common Englifh wild Clary, or Oculus Chrifti. In Davington and Ore Cburcb-Yards - not common.

May. $P$.

HOTTONIA. R. S. 285.

HOTTONIA paluftris. H. F. 72 .

Vivater Violet, or Gilliflower.

About the Decoy Ponds- uncommon. In Dykes near

Deal-common. June. P.

HYA 
HYACINTHUS anglicus. R.S. 373 .

HYACINTHUS non scriptus. H. F. 123.

Englinh Hyacinth, or Hare-bells.

In Woods and under Hedges-very common. April. P.

HYDROCOTYLE vulgaris. R.S, 22.

HYDROCOTYLE vulgaris. H. F. 96 .

Marnh Penny-wort, or White-rot.

In the Marhes about Luddenham and Ore - not uncommon. May. P.

HYOSCYAMUS vulgaris. R. S. 274.

HYOSCYAMUS niger. H. F. 77 .

Common Henbane.

By Road fides near the Town-common. June. P.

HYPERICUM. R. S. 342 .

HYPERICUM perforatum. H. F. 290.

Saint John's-wort.

Under Hedges, and by Roat fades - very common. July. P.

HYPERICUM Androfæmum diEtum. R.S. 343 . HYPERICUM birfutum. H. F. $29 \mathrm{I}$.

Tutfan, or hairy St. John's-wort.

In bollow Lanes in Boughton-uncommon. July. P. 


\section{( 49 )}

HYPERICUM Afcyron dictum caule quadran: gulo. R. S. 344 .

HYPERICUM quadrangulum. H. F. 292, Saint Peter's-wort.

In Davington Brooks-not uncommon. July. P.

HYPERICUM pulchrum Tragi. R. S. 342:

HYPERICUM pulcbrum. H. F. 290.

Upright Saint John's-wort.

In Byfing Wood - common. July. P.

HYPERICUM maximum Androfæmum vulgare dictum. R. S. 343 .

HYPERICUM Androsemum. H. F. $29 \mathrm{I}$.

Tutfan, or Park-leaves.

In a Hedge near Provender Wood uncommon. July. P.

HYPERICUM minus fupinum. R. S. 343 .

HYPERICUM bumifufum. H. F. 290.

The leaft trailing Saint John's-wort.

On fandy Lands in Ore-common. July. P.

HYPERICUM eleganifimum non ramofum folio lato. R. S. 343 .

HYPERICUM montenum. H. F. $29 \mathrm{r}$.

Imperforate Saint John's. wort.

In Byfing Wood - uncommon. July. P. 


\section{$(50)$}

J.

JACEA major. R. S. 198.

CENTAUREA Scabiosa. H. F. 325 .

Great Knap-weed, or Matfellon.

On the fides of Patbs - common. July. P.

JACEA nigra. R. S. 198.

CENTAUREA facea. H. F. 326 .

Common Knap-weed, or Matfellon.

On the fides of Fields-very common. Auguit. P.

JACOBÆA latifolia paluftris five aquatica.

R. S. 178.

SENECIO aquaticus. H. F. 317 .

Broad-leaved Marh or Water Ragwort.

In the Brents - common. Auguft. P.

JACOBÆA fenecionis folio incano perennis.

R.S. 177 .

SENECIO erucifolius. H.F. 377 .

Hoary Perennial Ragwort with Groundfel Leaves.

In Byfing Wood - not common. July. P.

JACOBEA vulgaris. R. S. 177 .

SENECIO facobaa. H. F. $3^{16 .}$

Common Ragwort.

In Meadowes and Paftures-very common. July. P. 


\section{(5 $\mathrm{r}$ )}

IRIS paluftris lutea. R. S. 374 :

IRIS Pseudacorus. H.F. 13.

Yellow Water Flower de luce.

In moift boggy Places - very common. June. P.

IRIS fylveftris quam Xyrim vocant. R. S. 375 .

IRIS fatidifima. H. F. I3.

- Stinking Gladdon.

By Sandgate Caftle near Folkftone-very uncommon.

July. P.

JUNIPERUS vulgaris baccis parvis purpureis.

R. S. 444 .

JUNIPERUS communis. H. F. 372 .

The common Juniper.

On dry chalky Commons - very common. May. S.

K.

KALI geniculatum perenne fruticofius procumbens. R. S. 136 .

SALICORNIA europea. H. F. 1. $\beta$.

Perennial Marh Sampire, or jointed Glafs-wort. In the Ine of Shepey-uncommon. Auguft. P.

KALI fpinofum cochleatum. R.S. 159.

SALSOLA Kali. H. F. 33 .

Prickly Glafs-wort.

On the Sbore between Graveney and Seafalter - not common. July. A. 


\section{$(52)$}

KNAWEL. R. S. 159 .

SCLERANTHUS annus. H. F. 160.

German Knot-grafs, or annual Knawel.

On barren dry fandy Soils-very common. Auguft. A.

\section{L.}

LACTUCA agnina feu Valerianella foliis ferratis.

R. S. $20 \mathrm{I}$.

VALERIANA Locufa. H. F. 12.

Small Corn Sallet, or Valerian with jagged leaves.

In Fields among Corn, and on old Walls — common. May. A.

LACTUCA fylveftris murorum. R. S. 162 . PRENANTHES muralis. H. F. 296.

Ivy-leaved wild Lettuce.

In Hedges near Wilderton-uncommon. - July. P.

LACTUCA fylveftris minima. R. S. 162.

LACTUCA faligna. H. F. 296.

The leaft wild Lettuce.

On Banks of Ditches near Sheernefs - uncommon. Auguft. A.

LAMIUM album. R. S. 240.

LAMIUM album. H. F. 225.

White Archangel or Dead Nettle. Under Hedges - very common. May. P. 


\section{( 53 )}

L.AMIUM rubrum. R. S. 240. LAMIUM rubrum. H. F. 225.

Red Archangel or Dead Nettle.

On Rubbith, and fides of Fields - very common. May. A:

LAMIUM cannabinum floribus albis verticillis purpurafcentibus. R. S. 24I.

GALEOPSIS Tetrabit. $\beta$. H. F. 226. White flowered Hemp-leaved Dead Nettle. In Hedges between Plomford and Badgen Downs not common. Auguft. A.

L A MIUM cannabino folio vulgare. R. S. 240. GALEOPSIS Tetrabit. H. F. 226.

Nettle Hemp, or Hemp-leaved Dead Nettle. In Byfing Wood-not common. Augurt. A.

LAMIUM cannabino folio, flore amplo luteo, labio purpureo. R. S. 241 .

GALEOPSIS Tetrabit $\delta$. H. F. 226. Hemp-leaved Dead Nettle with a parti-coloured Flower.

In Mr. Jacob's new Wood-bedge at Nackington very uncommon. Auguft. A.

LAviUM folio caulem ambiente majus et minus,

R. S. 240.

LAMIUM emplexicaule. H.F. 225 . Great Henbit. In Hop-grounds-very common. June. A. 


\section{( 54 )}

LAMIUM luteum. R. S. 242 .

GALEOPSIS Galeobdolon. H. F. 226.

Yellow Archangel, Dead Nettle, or yellow Nettle

Hemp.

In Woods and under ßady Hedges - very common. May. P.

LAMPSANA. R. S. 173.

LAPSANA communis. H. F. 303 .

Nipple-wort.

In moift Cornfields, and by Way-jides-very common.?

July. A.

I.APATHUM acetofum repens lanceolatum.

R. S. 143 .

RUMEX Acetosella. H. F. ${ }^{3} 6$.

Sheep's Sorrel.

On dry Sandy or gravelly Ground - very common.

July. P.

LAPATHUM acetofum vulgare. R. S. 143.

RUMEX Acetofa. H. F. 136 .

Common Sorrel.

In Meadowes and Paftures-very common. June. B.

LAPATHUM acutum. R. S. 142.

RUMEX acutus. H. F. 134 .

Sharp-pointed Dock.

In uncultivated Places-very common. June. P. 


\section{( 55$)$}

LAPATHUM acutum minimum. R. S. $14 \%$

An RUMICIS acuti varietas. H. F. 134 .

The leffer fharp-pointed Dock.

In Woods - not uncommon. June. P.

LAPATHUM folio acuto crifpo. R. S. $14 \mathrm{I}$.

RUMEX cripus. H. F. 134 .

Sharp-pointed Dock, with curled Leaves.

On the Mud Walls beyond the Sluice — common. July. P.

LAPATHUM folio acuto rubente. R. S. 142 .

RUMEX Sanguineus. H. F. 133 .

Blood-wort.

In Davington Brooks-uncommon. July. B.

LAPATHUM maximum aquaticum five Hydrolapathum. R.S. 140.

RUMEX. Britannica. H. F. 135 .

Great Water Dock.

In Ditcbes and boggy Places-very conmon. Auguft. P.

LAPATHUM pulchrum Bononienfe finuatum.

R. S. 142.

RUMEX pulcher. H. F. 134 .

Fiddle-Dock.

By the fides of Foot-ways about the Town - not uncommon. June. P. 
LAPATHUM viride. R. S. I 41 .

An RUMICIS acuti varietas, H. F. 134 .

The greener leaved Dock.

In frady Woods and moift Ground - not uncominon. June. $P$.

LAPATHUM vulgare folio obtufo. R. S. 141. RUMEX obtuffolius. H.F. 134 .

Common broad-leaved Dock.

In moift. Places, and by Road fides July. P.

LAPPA major Arctium Diofcoridis. R. S. 197. ARCTIUM Lappa. H. F. 304. Burdock, or Clot burr.

Under Hedges, and by Road-sides — very common. $\therefore$ Auguit. B.

LAPPA major capitulo glabro maximo. R.S. 196. ARCTIUM Lappa. $\beta$. H. F. 304.

Greater Burdock, with a larger Flower.

By Road-fides, and under Hedges _ common. Auguft. B.

LAPPA major capitulis parvis glabris. R.S. 197. ARCTIUM Lappa. H. F. 304.

Burdock with fmall Heads.

By-Road fides in Hern-hill —not uncommon. Auguft. B. 


\section{( 57 )}

LAPPA major montana capirulis tomentofis.

ARCTIUM Lappa. H. F. 304.

Greater Burdock with woolly Heads.

In the Way-fides near Luddenham Court-uncommon. Auguft. B.

LATHYRUS luteus fylveftris dumetorum. R. S. 320.

LATHYRUS pratenfis. H. F. 277.

Tare everlafting, or common yellow baftard:

Vetchling.

In Woods and Hedges - common. Augurt. A.

LATHYRUS major latifolius. R. S. 319.

LATHYRUS latifolius. H. F. 276 .

Broad-leaved everlafting Pea.

In a Field Hedge at Copton, and by the Road-fide near Boughton Street-uncommon. July. P.

LATHYRI majoris fpecies flore rubente et albido.

R. S. 319 .

LATHYRUS fylveftris. H. F. 276.

Narrow-leaved everlafting Pea.

In a Hedge of the Beacon Farm-uncommon. Aulguft. $P$.

LAUREOLA. R. S. 465.

DAPHNE Laureola.

Dwarf Laurel, or Spurge-Laurel.

In Woods and under Hedges - common. March. P. 


\section{( 58$)$}

LENS paluftris. R. S. 129.

LEMNA minor. H.F. 345 .

Duck's Meat.

In Ponds and fanding Waters - very common. June. $P$.

LENTICULA aquatica trifulca. R.S. 129.

LEMNA trifulca. H. F. 344.

Ivy-leaved Duck's Meat.

In the Ditches of Ham Marh-common. June. P.

LENTICULA paluftris major. R. S. I 29.

LEMNA polyrbiza. H. F. 345 .

Greater Duck's Meat.

In a Ditch near Waterham-uncommon. July. A.

LEPIDIUM latifolium. R. S. 304 .

LEPIDIUM latifolium. H.F. 244.

Dittander, or Pepperwort.

Near the King's Head Key in the Town-very uncommon. July. P.

LEUCANTHEMUM vulgare. R. S. 184.

CHRYSANTHEMUM Leucantbemum. H. F:

321.

The greater Daifie, or Ox-eye.

In Paftures, and amongft Corn-common. May. P.

LEUCO. 


\section{(59)}

LEUCOJUM luteum vulgo Cheiri flore fimplici.

R.S. 291.

CHEIRANTHUS Cbeiri. H.'F. 250.

Wall-flower, or wild Chier.

On old Walls - very uncommon. May. P.

* LEUCOJUM bulbofum trifolium minus.

Bauhini Pinacis. 56.

GALANTHUS nivalis. Lin. Syft. Natur.

II. p. 234.

The Snow Drop.

In Paftures at South-ftreet, and at Davington very common. Febriary. $P$.

LIGUSTRUM. R. S. 465 .

LIGUSTRUM vulgare. H.F. 3 .

Privet, or Prim.

In Woods and Hedges - very common. May. S.

LILLIUM convallium. R. S. 264.

CONVALLARIA majalis. H. F. 126.

Lilly Convally, or May Lilly.

In the Blean Woods - plentifully. May. P.

LIMNOPEUCE. R. S. 136 .

HIPPURIS vulgaris. H.F. I.

Mare's Tail.

In the Powder-Mill Waters-uncommon. May. P.

LIMO- 


\section{( 60$)$}

LIMONIUM. R. S. 200.

STATICE Limonium. H. F. II4.

Sea Lavender.

In the Salt Marßhes-very common. Auguft. P.

LINARIA Elatine dicta folio acuminato.

R. S. ${ }^{*} 282$.

ANTIRRHINUM Elatine. H. F. 237.

Sharp.pointed Fluellin.

In Corn Fields - common. September. A.

LINARIA Elatine dicta folio fubrotundo. R. S. ${ }^{*} 282$.

ANTIRRHINUM fpurium. H. F. 237.

Round- leaved Fluellin.

In the Corn fields of Prefton-not uncommon. Auguft. A.

LINARIA lutea vulgaris. R. S. *28I. ANTIRRHINUM Linaria. H. F. $23^{3}$.

Common yellow Toad-flax.

On the fides of Fields and Roads - very common. July. P.

LINUM fylveftre anguftifolium floribus dilute purpurafcentibus vel carneis. R. S. $3^{62}$. LINUM tenuifolium. H. F. 116.

Narrow-leaved Wild Flax.

Upon Beacon Hill - not common. June. P. 


\section{$(6 !)$}

LINUM fylveftre cæruleum perenne ereetius flore et capitulo majore. R. S. 362 . LINUM perenne. H. F. 115 .

Perennial Blue Flax.

In the Ine of Shepey - uncommon. June. P.

LiNUM fylveftre catharticum. R. S. 362 .

LINUM catbarticum. H. F. II6.

Purging Flax, Wild Dwarf Flax, Mill-mountain On fandy and chalky Soils-very common. June. A.

LITHOSPERMUM, feu Milium Solis. R.S. 228.

LITHOSPERMUM officinale. H. F. 65.

Gromwell, Gromil, or Graymill.

In Badgen Wood, and by Road fides-not uncommon: June. $P$.

LONCHITIS afpera. R, S. I18.

OSMUNDA Spicant. H. F. 382.

Rough Spleen-wort.

In the Woods necir Dunkirk-uncommon. July. P.

LOTI corniculatæ major fpecies. R. S. 334 . LOTUS corniculata. H. F. 288. $\gamma$. The greater Bird's-foot Trefoil. Under Hedges near Byfing Wood not common. July. P. 


\section{$(62)$}

LOTUS corniculata glabra minor. R. S. 334 . LOTUS corniculata. H. F, 288.

Bird's-foot Trefoil.

Under Hedges, and in Fields in Ofpringe - common. July. P.

LUPULUS mas et fømina. R. S. 137 .

HUMULUS Lupulus. H. F. 369 .

Hops, the male and female.

In Hedges in Prefton - not uncommon. July. P.

LUTEOLA. R. S. 366 .

RESEDA Luteola. H. F. $18 \mathrm{r}$.

Wild Woad, Yellow Weed, or Dyers Weed.

In Davington Cburcb-Yard, and on barren Landsnot uncommon - June. A.

LYCHNIS major noetiflora Dubrenfis perennis. R. S. 340 .

CUCUBALUS vifcosus. H. F. $16_{3}$.

Dover Campion.

On the Cliffs between St. Margaret's and Dover plentifully. July. P.

LYCHNIS maritima repens. R. S. 337 .

SILENE amona.? H. F. 164.

Sea Campion.

In the Ine of Shepey. - uncommon. Auguft. P. 
LYCHNIS plumaria fylveftris fimplex. R.S. 338.

LYCHNIS Flos cuculi. H. F. 174.

Meadow Pink, Wild Williams, Cuckow Flower. In moift Pafures - very common. June. P.

LYCHNIS Saponaria dicta. R. S. 339 .

SAPONARIA officinalis. H. F. 160.

Common Soap-wort.

In Hedges near Sindal and Keatings, and beyond Sheldwich Church - uncommon. July. P.

LYCHNIS fegetum major. R. S. 338. AGROSTEMMA Gitbaco. H. F. 173 . Cockle.

In Corin Fields - common. June. A.

LYCHNIS fupina maritima Ericæ facie. R.S. 338 . FRANKENIA levis. H. F. IIg.

Smooth Sea-Heath.

Near Minfter in Shepey-uncommon. Auguft. P.

LYCHNIS fylvertris anguftifolia caliculis turgidis ftriatis. R. S. $34 \mathrm{I}$.

SILENE conoidea. H. F. 165.

Narrow-leaved Campion.

On the Sandbills near Deal - uncommon. July.
G 2
LYCH- 


\section{( 64 )}

LYCHNIS fylveftris flore albo. R. S. 339.

LYCHNIS dioica. H. F. 174.

Wild white Campion.

In Woods and Hedges - not uncommon. June. P.

LYCHNIS fylveftris flore rubello. R.S. 339 .

LYCHNIS dioica. H. F. I74. $\beta$.

Red flowered wild Campion.

In Woods and Hedges - common. June. 'P.

LYCHNIS fylveftris, quæ Behen album vulgo.

R. S. 337 .

CUCUBALUS Beben. H. F. 163 .

Spatling Poppy, Bladder Campion, or white

Corn Campion.

In dry Paftures, and in Corn fields-common. July. P.

LYCOPUS paluftris glaber. R. S. 2.36 .

LYCOPUS earopcus. HI. F. 8.

Water Horehound.

On the Banks of the River, and in boggy Places common. July. P.

İYSIMACHIA campeftris. R. S. $3 \%$.

EPILOBIUM montanum. H. F. $14 \mathrm{I}$.

The greater fmooth-leaved Willow-herb, or

Loofe-ftrife.

By the fides of Ditches, and under moift Hedges -
common. July. B. 
LYSIMACHIA lutea. R. S. 282.

\section{$\left(6_{5}\right)$}

LYSIMACHIA vulgaris. H.F. 72 .

Yellow Willow-herb, or Loofe-frife.

On the fwampy Ground near Stone Bridge - very uncommon. Augurt. P.

LYSIMACHIA filiquofa glabra media five minor.

R. S. 3 II.

EPILOBIUM tetragonum. H. F. I4r.

Middle fmooth-leaved Willow-herb, or Loofeftrife.

In moift Ditcbes, and near the River common. July. P.

LYSIMACHIA filiquofa glabra minor anguftifolia. R.S. $31 \%$.

EPILOBIUM painftre. H. F. $14 \mathrm{I}$.

The leaft fmooth codded Willow-herb, or Loofeftrife.

In mojt Places - common. July. P.

LYSIMACEIA filiquofa hirfuta magno flore.

R. S. 3 II.

EPLLOBIUM ramofum. H. F. $14 \pi$.

Great flowered Willow herb, called Codlings and

Crtam.

On the fides of the thooting Meadow Stream-common. July. P.

LYSIMACHIA filiquofa hirfuta parvo flore. R. S. SII.

EPILOBIUM birfutum. H. F. 140. The leffer hairy codded Willow-herb, or Loofeftrife.

On the fades of the hooting Meadow Stream-common. July. P. LYSI- 


\section{$(66)$}

LYSIMACHIA fpeciofa, quibufdam Onagra

dicta. R. S. 310.

EPILOBIUM angufifolium. H. F. 140.

Rofebay Willow-herb.

In a Wood Hedge beyond the five Mile Stone to Charing

- very uncowmon. July. P.

M.

MALUS fylveftris. R. S. $45^{2}$.

PYRUS Malus. H. F. 189 .

The Crab Tree.

In Hedges - not uncommon. May. S.

MALVA fylveftris minor. R. S. 25I.

- MALVA rotundifolia. H. F. 268.

Small wild or Dwarf Mallow.

On dry Banks by Road fides common. July to Oetober. A.

MALVA vulgaris. R. S. $25 \mathrm{r}$.

MALVA fylveftris. H. F. 268.

Common Mallow.

Under Hedges, by Way fides, and on Rubbifs - very common. July: B.

MARRUBIUM album. R. S. 239.

MARRUBIUM vulgare. H. F. 228.

White Horehound.

On Sheldwich Lees - not common. July. P. 
MATRICARIA. R. S. 187 .

MATRICARIA Partbenium. H.F. $32 \mathrm{t}$.

Feverfew.

Under Hedges, and on old Walls-comman. June. P.

MELAMPYRUM fylvaticum flore luteo. R. S. * 286 .

MELAMPYRUM fylvaticum. H. F. $2{ }_{3} 6$.

Common Cow-wheat with a yellow Flower.

In Byfing Wood-very common. June to A uguft. A.

MELILOTUS vulgaris. R. S. $33 \mathrm{I}$.

TRIFOLIUM Melilotus officinalis. H. F. 282.

Common Melilot.

Amongft the Corn at Halfo-plentifully. July. B.

* MELiss A officinalis. Miller Botan. Off. 290. MELISSA officinalis. Lin. Syftem. Nat. II.

p. 400.

Common Garden Balm.

Under Hedges in Boughton and Davington - uncommon. July. P.

MENIANTHES paluftre triphyllum latifolium et anguftifolium. R. S. 285.

MENYANTHES trifolia. H. F. $7 \mathrm{r}$.

Marth Trefoil, or Buckbean.

In the moift Meadores of the Abbey-commorn. July. P. 


\section{(68)}

MENTHA aquatica feu Syfmbrium. R. S. 233. MENTHA aquatica. H. F. 223.

Water-mint.

In moift Places, and by the River fide - very common. Augutt. P.

MENTHA feu Calamintha aquatica. R. S. 232. MENTHA arvenfis. H. F. 223.

Water-mint with whirled coronets.

On the fides of Ditches, and in Corn felds - common. Anguft. P.

MENTHA fpicata anguftifolia glabra fpica latiore. R. S. 234.

MENTHA Spicata. H. F. 221.

Spear-mint.

On the Side of the River oppofite the Powder Stove very uncommon. A uguit. P.

MENTHASTRUM folio rugofo rotundiore fpontaneum flore fpicato odore gravi. R. S. 234. MENTHA rotundifolia. H. F. $22 \mathrm{I}$.

Round leaved Horfe-mint.

In a Meadow bebind King's Mill - uncommon. Auguft. P.

MENTHASTRUM fpicatum folio longiore candicante. R.S. 234.

MENTHA longifolia. H. F. 22I.

Long leaved Horfe-mint.

On the Banks of the River above Ofpringe Church not uncommon. Auguft. $P$.

MER- 


\section{$(.69)$}

MERCURIALIS annua glabra vulgaris, Mercurialis mas et Foemina. R. S. 139.

MERCURIALIS annua. H. F. $37 \mathrm{r}$.

French Mercury.

On Rubbih and in Gardens - not common. September. A.

MERCURIALIS perennis repens. Cynocrambe dieta. R. S. 138.

MERCURIALIS perennis. H. F. $37 \mathrm{I}$.

Dog's Mercury.

In Woods and under Hedges-very common. A pril. P.

MESPILUS A pii folio fylvettris fpinofa, five Oxyacantha. R. S. 453 .

CRAT EGUS Oxyacantba. H. F. I88. The white Thorn, or Hawthorn, called Quick. In Woods and Hedges - very common. May. S.

MESPILUS Apii folio fylveftris non fpinofa.

R. S. 453.

CRAT ÆGUS torminalis. H. F. 188.

The common or wild Service-tree, or Sorb.

In the Woods about Chart - uscommon. A pril or

May. S.

MILLEFOLIUM vulgare. R. S. 183 .

ACHILLEA Millefolium. H. F. 324 .

Common Yarrow, Millfoil, or Nofe-bleed.

In Meadowes and Paftures - very common. June. P. 


\section{( 70$)$}

MOLLUGO montana minor Gallio albo fimilis. R.S. 224.

GALIUM montanum. H. F. 56 .

Small mountain baftard Madder, or mountain

Ladies Beditraw.

In the Corn fields about Sindal-not uncommon. July. P.

MOLLUGO vulgatior. R. S. 223.

GALIUM Molugo. H. F. 56 .

Wild Madder, or great baftard Madder.

In Hedges and bufay Places-very common. July. P.

MOLLUGINIS vulgatioris varietas minor.

R. S. 224.

GALIUM paluftre. H. F. 57.

White Ladies Bediftraw.

On the fides of the fhooting Meadow Stream-common. July. P.

MOSCHATELLINA foliis fumarix bulbofæ.

R.S. 267.

ADOXA Mofcbatellina. H. F. 150.

Tuberous Mofchatell.

In Woods and Hedges-very common. April. P.

MYOSOTIS fcorpioides hirfuta. R. S. 229.

MYOSOTIS fcorpioides. H. F. 65 .

Moufe-ear Scorpion grafs.

In Woods, on old Walls and dry Paffures_common. April to Auguft. P.

MYOSO. 


\section{(7I)}

MYOSOTIS fcorpioides paluftris. R. S. 229. MYOSOTIS fcorpicides. H. F. $65 . \delta$.

Water Scorpion-grafs.

On the fides of Ore Mill-pond-not common. April to Auguif. P.

MYRRHIS fylveftris feminibus afperis. R.S. 220. SCANDIX Antbriccus. H. F. 108.

Small Hemlock-Chervil with rough Seeds. Under Hedges, and by the fiales of Ditches — common. May. A.

N.

NAPUS fylveftris. R. S. 295 .

BRASSICA Napus. H. F. 253.

Wild Navew.

Amongf Corn, ana' by the fides of Ditches— common. May. B.

NARCISSUS fylveftris pallidus calyce luteo. R.S. 371 .

NARCISSUS Pseudo Narcifus. H. F. 123.

Wild Englin Daffodil.

In the Orcbards near Selling-Street-very uncommon. April. P.

NASTURTIUM fupinum capfulis verrucofis, five Coronopus Ruellii. R. S. 304. COCHLEARIA Coronopus. H. F. 248. Swine's Crefs.

On the fides of Roads and Foot-patbs - very common. July. A.

NEPETA 
NEPETA major vulgaris. R. S. 237.

NEPETA Cataria. H. F. 220.

Nep, or Cat-mint.

Under the Weft Hedge of King's. Field - not common.

July. P.

NUMMULARIA. R. S. 283.

LYSIMACHIA Nummularia. H.F. 73.

Money-wort, or Herb Two-pence.

In moift Hedges at Luddenham, and near Provender - not common. June. P.

NUMMULARIA ninor flore purpurafcente.

R. S. 283.

LYSIMACHIA tenella. H. F. 73 .

Purple flowered Money-wort.

In moifs Meadows by the Abbey - not uncommon. July. P.

NYMPHÆA alba. R. S. 368.

NYMPHÆE alba. H. F. 206.

White Water Lilly.

In Ham Ponds near Sandwich-uncommon. July. P.

NYMPHÆA lutea. H. F. 368.

NYMPHÆA lutea. H. F. 206.

Yellow Water Lilly.

In Ore Mill Pond - uncommon. July. P. 


\section{$(73)$}

O.

OENANTHE aquatica. R. S. 210.

UENANTHE fifuloja. H. F. 104.

Water Dropwort.

In the Ditches near Fairbrook-common. July. P.

OENANTHE Cicutæ facie. Lobellii. R.S. 210.

OENANTHE crocata. H. F. $10 j$.

Hemlock Dropwort.

In the Water-lane between Sitingbourn and Miltonvery uncommon. June. $\mathrm{P}$.

ONOBRYCHIS feu Caput gallinaceum. R.S. 327 . HEDYSARUM Onoorycbis. H. F. 281 .

Suintfoin or Cock's-head.

On the cbalky Hills - common. June. P.

OPHIOGLOSSUM. R. S. I 28.

OPH:OGLOSSUM vulgatum. H. F. 382 .

Adders Tongue.

In Meadores at Ofpringe and Luddenham - not uncommon. May. P.

OPULUS Ruellii. R. S. $4^{\text {fo. }}$

VIBURNUM Opulus. H. F. II 2.

Water Elder, or Cherrywood.

In Badgen Wood, and in Hedges - not uncommon.

June. $S$. 


\section{$(74)$}

ORCHIS abortiva rufa five Nidus avis. R. S. 382 . OPHRYS Nidus avis. H. F. $33^{8}$.

Birds-neft Orchis.

In Byfing and Jud's Woods - very uncommon. June. $P$.

ORCHIS alba bifolia minor calcari oblongo.

R. S. 380.

ORCHIS bifolia. H. F. 333 .

Butterfly Orchis.

In Badgen and Cockfet Woods - not common. May. P.

ORCHIS anthropophora oreades. R. S. 397.

OPHRYS antbropopbora. H. F. 340.

The Green Man Orchis.

On cbalky Banks - common. June. P.

ORCHIS fuciflora galea et alis purpurafcentibus.

R. S. 379.

OPHRYS apifera. H. F. 340.

The Bee Orchis.

In Meadows in Ofpringe-not uncommon. June. P.

ORCHIS galea et alis fere cinereis. R.S. $3 ; 8$.

ORCHIS militaris. H. F. 335 .

The Man Orchis.

On cbalky bußhy Banks beyond Whitehill, Ofpringe not common. June. P. 



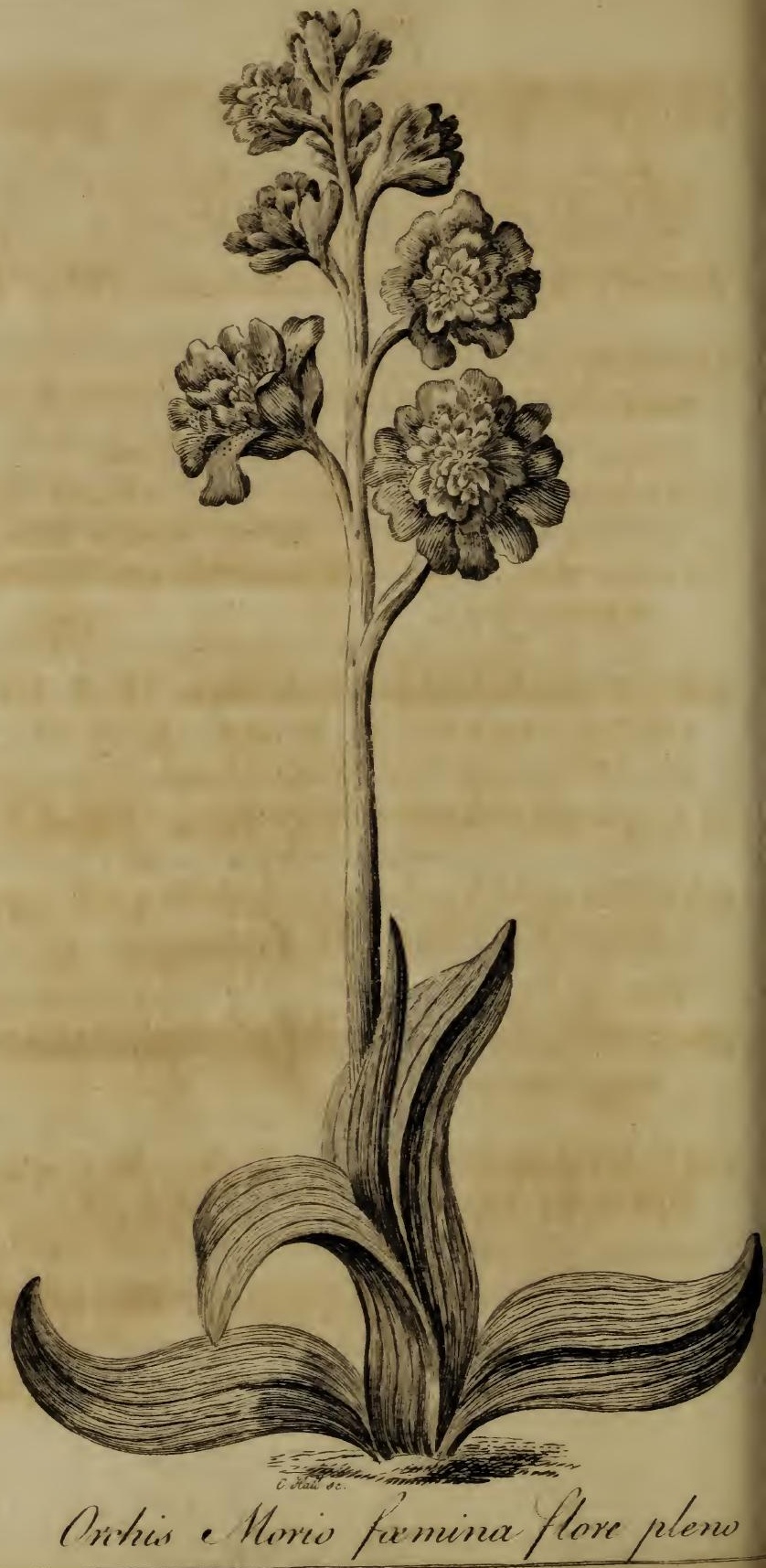




\section{( 75$)$}

ORCHIS magna latifolia galea fufca vel nigricante. R. S. 378 .

ORCHIS purpurec. H. F. 334 .

The purple Man Orchis.

In Cockfet and Jud's Woods-uncommon. May. P.

ORCHIS morio fœmina. R. S. 377 .

ORCHIS morio. H. F. 333 .

Female Orchis, or Female Fools Stones.

In Meadowes - very comwon. In a Meadow near Cades in Ofpringe, fome of a Rofe Colour, fome white, and a very ferw with double Focrevrs. May and June. P.

ORCHIS morio mas foliis maculatis. R. S. 376 .

ORCHIS mascula. H. F. 333 .

Male Orchis, or Male Fools Stones.

In Hedges and Meadows-very common. May. P.

ORCHIS myodes galea et alis herbidis. R.S. 379 . OPHRYS muscifera. H. F. 340.

The Fly Orchis.

Among the Bußbes in Ofpringe Parfonage Meadoresnot uncommon. June. $P$.

ORCHIS odorata mofchata. K. S. 378 .

OPHRYS Monorchis. H. F. 339 .

Yellow or Mufk Orchis.

On the cbalky Banks of Ofpringe Parfonage Meadows - uncommon. June. P. 


\section{( 76$)$}

ORCHIS palmata minor flore luteo-viridi.

R.S. 381 .

SATYRIUM viride. H.F. 337.

Frog Satyrion or Orchis.

On dry graffy Banks near Whitehill

uncommon. June. $P$.

ORCHIS palmata pratenfis latifolia longis calcaribus. R. S. 380.

ORCHIS latifolia. H. F. 335 .

Male handed Orchis, or Satyrion royal.

On moift boggy Grounds about Ore Mill —— not uncommon. June. P.

ORCFils palmata fpeciofiore thyrfo, folio maculato.

R. S. 38 .

ORCHIS maculata. H. F. 335 .

Female handed Orchis, or Satyrion royal.

In Cba!k Pits - common. June. P.

ORCHIS pannonica. R. S. 377 .

ORCHIS uftulata. H. F. 334.

Little purple flowered Orchis.

On the South fde of Chatham Hill _ pientifully.

May. P.

ORCHIS purpurea fpica congefta pyramidali.

. R. S. 377 .

ORCHIS pyramidalis. H.F. 334 .

Purple late flowering Orchis.

On sbalky Eanks in Ofpringe - common. July. P. 
ORCHIS firalis alba odorata, five Triorchis. R. S. 378 .

OPHRYS fpiralis. Triple Ladies Traces.

In Ofpringe Parfonage Meadows - uncommon. September. $P$.

ORCHIS five Tefticulus fphegodes hirfuto flore. R. S. 380.

OPHRIS apifera. H. F. 340, $\beta$.

Humble Bee Orch is with green Wings.

On the Banks of Whiteiill in Selling - uncommon. April. P.

ORIGANUM Onites. R. S. 236 .

ORIGANUM Onites. H. F. 229.

Pot Marjoram.

In Walderhare Park-very uncommon. Auguft. P.

ORIG ANUM vulgare fpontaneum. R. S. 236 . ORIGANUM vulgare. H.F. 229.

Wild Marjoram.

Under Hedges, and in bufby Places - very common. July. $\mathbf{P}$.

ORNITHOPODIUM radice nodofa. R.S. 326 . ORNITHOPUS perpufillus. H. F. 280. Birds Foot.

In Broom fields at Hernhill-common. May to Auguft. P. 


\section{$(78)$}

OROBANCHE major Garyophyllum olens.

R. S. ${ }^{*} 288$.

OROBANCHE major. H. F. 232 .

Broom-rape.

In Broom fields - not uncommon. June. B.

OROBUS fylvaticus foliis oblongis glabris.

R. S. 324 .

OROBUS tujerofus. H. F. 274.

Wood Pea, or Heath Pea.

In Jud's Wood - very common. May. P.

OSTRYA Ulmo fimilis, fructu in umbilicis foliaceis.

R. S. $45^{\mathrm{r}}$.

CARPINUS. Betulus. H. F. 360 .

The Horn-beam Tree, or Horfe-Beech.

In Woods - common. May. S.

OXYS alba. R. S. ${ }^{*} 28 \mathrm{r}$.

OXALIS Acetosella. H. F. 173.

Wood Sorrel.

In Byfing and Jud's Woods - common. June. A.

$$
\text { P. }
$$

PAPAVER corniculatum luteum. R. S. 309. CHELIDONIUM Glaucium. H. F. 202.

Yellow horned Poppy.

On the Beacb at Sea-Salter-common. July. A.

PAPAVER 


\section{(79)}

PAPAVER corniculatum luteum. Chelidonia dictum. R. S. 309.

CHELIDONIUM majus. H.F. 20 r.

The greater Celandine.

Annong Rubbifh, and on old Walls-common. June. P.

PAPAVER laciniato folio capitulo breviore glabro annuum, Rhœas dictum. R. S. 308.

PAPAVER Rbicas. H. F. 203.

Red Poppy, or Corn Poppy.

In Cornfields - very common. July. A.

PARIETARIA. R. S. 158.

PARIETARIA officinalis. H. F. 376.

Pellitory of the Wall.

On old Walls - very common. July. P.

PARONYCHIA vulgaris. R. S. 292 :

DRABA verna. H. F. 243 .

Common Whitlow Grafs.

On old Walls and dry Paftures - very common. April. A.

PASTINACA fylveftris latifolia. R. S. 206. PASTINACA fativa. H. F. Iog.

Wild Parfnep.

On the cbalky Bank by the Roadfide near Wildertonuncommon. July. B.

PEDI- 


\section{( 80$)$}

PEDICUI_ARIS feu Crifta galli lutea. R. S. * 284.

RHINANTHUS Crifta galli. H. F. 234 .

Yellow Rattle, or Cocks-comb.

InByfing Wood, and by Roadfides-common. July. A.

PEDICULARIS paluftris rubra elatior. R. S. * 284.

PEDICULARIS paluftris. H. F. 236 .

Great Marh red Rattle, Cocks-comb, Loufe. wort.

About the Powder Mills at Ore-not common. June. A.

PEDICULARIS pratenfis rubra vulgaris. R. S. * 284.

PEDICULARIS fylvatica. H. F, 236 .

Red Rattle, Cocks-comb, Loufe-wort.

In Jud's Wood - common. June. P.

PENTAPHYLLOIDES Argentina dicta. R. S. 256.

POTENTILLA Argentina. H. F. 195.

Wild Tanfey, or Silver-weed.

By the fades of Roads'and Patbs-common. July. P.

PENTAPHYLLUM erectum foliis profunde

fectis fubtus argenteis flore luteo. R. S. 255 .

POTENTILLA argentea. H. F. 196.

Tormentil Cinquefoil.

In a Road bedge near Newnham.uncommon. May. P

PENIA- 


\section{$(81)$}

PENTAPHYLLUM vulgatifimum. R. S. $225^{\circ}$ POTENTILLA reptans. H. F. 197.

Common Cinquefoil, or Five-fingered Grafs. By the fides of Roads and Patbs-common. June. P.

PENTAPTEROPHYLLON aquaticum, forculis ad foliorum nodos. R. S. 315.

MYRIOPHYLLUM verticillatum. H. F. 357 . Verticillated Water-millfoil.

In the Ditches of Graveney Marih - not uncommon. July. P.

PERCEPIER Anglorum. R. S. 159. APHANES arvenfis. H. F. 60 .

Purney-piert.

Upon barren Lands and old Walls-common. May. A.

PERSICARIA latifolia geniculata caulibus marulatis. R. S. 145.

POLYGONUM Perficaria. H. F. $147 \cdot \beta$.

Dead or fpotted Arfmart.

In Fields, and on Rubbis - common. Auguft. A.

PERSICARIA maculofa. R. S. 145.

POL.YGONUM Perficaria. H. F. 147,

Dead or fpotted Arfmart.

In Meadowes and Ditches - very common. Sept. A. 
PERSICARIA mitis major foliis pallidioribus. R.S. 145.

POLYGONUM penfylvanicum. H. F. 148.

Greater dead Arfmart with pale Leaves.

On a Dungbill at Staple Street - uncommon. Auguft. A.

PERSICARIA falicis folio perennis, Potamogiton anguftifolium dicta. R. S. 145. POLYGONUM ampibibium. H.F. 147. Perennial Willow-leaved Arfmart.

On the fides of the Abby Ditches — not common. July. $\mathbf{P}$.

PERSICARIA vulgaris acris, feu Hydropiper. R. S. 144 .

POLYGONUM Hydropiper. H. F. 148. Lakeweed, Arimart, Water-pepper.

In moift Places and wet Ditcbes very common. Auguit. A.

PETASITES. R. S. 179.

TUSSILAGO Petafites. H.F. 315 .

Common Butter Bur, Peftilent-wort.

Near Waterham in Hernhill-uncommon. March. P.

* Petroselinum vulgare. Miller. Botan. Officinal. 324 .

APIUM Petrofelinum. Linnæi Syftem. Natur. II. p. 217. Common Parney.

On the Weft Church-yard Wall — very uncommon. June. B. 


\section{$(83)$}

PEUCEDANUM. R. S. 206.

PEUCEDANUM officinale. H. F. 108:

Hog-fennel, or Sulphur-wort.

On the Wall leading to Thorn Creek — plentifully. June. $P$.

PHELLANDRIUM vel Cicutaria aquatica quorundam. R. S. 215.

PHELLANDRIUM aquaticum. H. F. $10 \%$

Water Hemlock.

On boggy Ground between Staple-Street and Herno hill Church-uncommon. July. B.

PHYLLITIS feu Lingua cervina vulgo. R.S. I 6 . ASPLENIUM Scolopendrium. H. F. 384 .

Hart's Tongue.

In Bady Lanes, bollow Ways, and on Walls-com. mon. $\mathrm{F}$.

PILOSELILA repens. R. S. 170.

HIERACIUM Pilosella. H. F. 298.

Common creeping Moufe ear.

On dry fandy Banks - common. May. A.

PIMPINELLA Saxifraga. R. S. 213.

PIMPINELLA major. H. F. 110.

Great Burnet-faxifrage.

In a tbick Hedge beyond Whitehill - uncomnon. Auguft. P. 


\section{$(84)$}

PIMPINELLA Saxifraga minor. R. S. 213 .

PIMPINELLA Saxifraga. H. F. 111. $\beta$.

Small Burnet-faxifrage with divided Leaves.

In Meadorws in Hernhill — not uncommon. Auguft. P.

PIMPINELLA Saxifraga minor foliis fanguiforbæ. R. S. 213.

- PIMPINELLA Saxifraga. H. F. im.

The leffer round leaved Burnet-faxifrage.

In dry Pafures - common. Auguft and September. $P$.

PLANTAGO aquatica. R. S. 257 .

ALISMA Plantego. H. F. $137^{\circ}$.

Great Water Plantain.

In moift Places, and on the fides of Ponds common. Augutt. P.

PLANTAGO foliis laciniatis, Coronopus dieta.

PLANTAGO Coronopus. H. F. 52.

Buck's-horn Plantain, Star of the Earth.

In the Abby Meadows - very conmon. Auguft. A.

PLANTAGO latifolia vulgaris. R. S. 314.

PLANTAGO major. H. F. 5 I.

Great Plantain, Way-bread.

By Way fides - very common. July. P. The Plantago panicula fparfa, found in Perry fieid, is an elegant variety of this Plant, but very uncommon. 


\section{$(85)$}

PLANTAGO major incana. R. S. 314. PLANTAGO media. H. F. 5 I.

Hoary Plantain.

By Roadfides - common. July. P.

PLANTAGO marina. R. S. 315 . PLANTAGO Loefingii. H. F. 52 .

Sea Plantain.

On the fides of the Ditches and Sea Wails - common. July. P.

PLANTAGO maritima. H. F. 52 .

Narrow leaved Plantain.

Iil the Brent Marhes-not uncommon. July. P.

PLANTAGO quinquenervia. R. S. 314.

PLANTAGO lanceolata. H. F. 52.

Rib-wort, or Rib wort Plantain.

Iis Fields and Meadows - very comimen. June to Auguit. P.

POLYGALA. R. S. * 287 .

POLYGALA vulgaris. H. F. 271.

Milk-wort.

In dry Fie'ds, and under Hedges_- very common. June. $P$. 


\section{$(86)$}

POLYGONUM brevi anguftoque folio. R.S 146 .

POLYGONUM aviculare. H. F. 149. $\beta$.

Knot-grafs with a finall narrow Leaf.

hy Road fides-common. June to September. A.

POLYGONUM mas vulgare. R. S. 145.

POLYGONUM aviculare. H. F. 149.

Common Knot-grafs.

In uncultivated Ground, and by Way fides - very

common. June to Oetober. A.

POLYGONUM oblongo anguftoque folio.

R. S. 146 .

POLYGONUM aviculare. H. F. 149. $\gamma$.

Knot-grafs with a more oblong narrow Leaf.

In Corn fields at Hernhill - common. June to September. A.

POLYPODIUM. R. S. 117.

POLYPODIUM vulgare. H. F. 387 .

Common Polypody.

In Byfing Wood, and on Banks of bollow Ways common. P.

POPULAGO; five Caltha paluftris. R. S. 272. CALTHA palifti is. H. F. 214 .

Marh Marigold.

In the Brent Marfhes-very common. April. P. 


\section{$(87)$}

POPULUS aiba. R. S. $4+6$.

POPULUS alba. H. F. 370 .

The White Poplar, or Abele Tree.

In miift Woods, and boggy Places - common. March. S.

POPULUS I. ybica Plinii. R. S. $44^{6}$.

POPULUS tremula. H. F. 370.

The Afp, or Trembling Poplar.

In moift Places in Hernhill not uncommon. March. S.

POPULUS major. R. S. $44^{6 .}$

POPULUS nigra. H. F. 370 .

The Black Poplar.

In moitt boggy Places in Hernhill - not uncominon. March. S.

PORTULA. R. S. 368.

PEPLIS Portulaca. H. F. 128.

Water Purnane.

In the Brent Meadows - not uncommon. September. P.

POTAMOGITON foliis pennatis. R.S. 150 . MYRIOPHY LLUM Jpicatum. H. F. 357 .

Feathered or fpiked Water Millfoil. In the Powder Mill Waters, and Graveney Marn Dykes-common. July. P. 


\section{( 88$)$}

POTAMOGITON feu Fontalis crifpa. Tribulus

aquaticus. R. S. 149.

POTAMOGETON crispum. H. F. 6I.

The great r Water Calirops.

In the Powder Mill Waters-common. June. P.

POTAMOGETON feu Fontalis media lucens.

R. S. 149 .

POTAMOGETON ferratum. H. F. $6 \mathrm{I}$.

The lefier Water Caltrops, or Frog's Lettuce.

In the River near the Powder Stove-not uncommen. June. P.

POTAMOGITON gramineum latiufculum foliis et ramificationibus denfe fipatis. IR. S. 149 . POTAMOGETON granineum. H.F. 62.

Grafs leaved Pond weed.

In the fallow Waters about the Powder Mills - not coinincn. P.

POTAMOGITONnaritimu'n ramofifimum grandiufculis capitulis, capillaceo folio noftras. R.S. 150.

PO ГAMOGETON marinum. H. F. 63 . Sea Pond-weed.

In Dykes near Sheernefs - uncommon. Auguft. P.

POT AMOGITON millefolium, feu foliis gramineis ramofum. R. S. 150. POTAMOGETON pestinatum. H. F. 62. Fine or Fennel-leaved Pond-weed. In Ofpringe River - not common. June. P. 
POTAMOGITON perfoliatum. R.S. 149. POT AMOGETON perfoliatum. H. F. 60. Perfoliated Pond-weed. In Ofpringe River - common. July. P.

POTAMOGITON rutundifolium. R.S. 148 . POTAMOGETON natans. H. F. 60.

Broad leaved Pond-weed.

In a Pond near Uplees - uncommon. Auguft. P.

PRIMULA pratenfis inodora lutea. R. S. 284. PRIMULA veris. H. F. 70. $\beta$.

Great Cownips, or Oxnips.

In thick Hedges beyond Whitehill - not common. April. P.

PRIMULA veris major. R. S. 284.

PRIMULA veris. H. F. 7r, $\alpha$.

Common Pagils, or Cownlips.

In Meadorws and Hedges-very common. A pril. P.

PRIMULA veris vulgaris. R. S. 284.

PRIMULA rulgaris. H. F. 70.

Common Primrofe.

In Woods and Hedges - very common. February to May. P.

$$
\text { I } 3 \quad \text { PRU. }
$$




\section{(90)}

PRUNELLA. R. S. 238.

PRUNELLA vulgaris. H. F. 231.

Common Self-heal.

In Meadows - common. Auguift. P.

PRUNUS fylveftris. R.S. 48.

PRUNUS pinosa. H. F. 186.

The Black Thorn, or Sloe Tree.

In Hedges - very common. A pril. S.

PRUNUS fylveftris major. R. S. 462 .

PRUNUS infititia. H. F. 186.

The black Bullace Tree.

In Some Hedges near Rickefey-Lane in Selling uncommon. April. S.

PRUNUS fylveftris fruttu majore albo. R.S. 462.

PRUNUS infititia. H. F. 186. $\gamma$.

The white Bullace Tree.

In Hiea'ges at Cades in Ofpringe, and at Selling uncominor. April. S.

PRUNUG fylveftris fruetu rubro acerbo et ingrato.

R. S. $4{ }^{6}$.

PRUNUS infititia. H. F. 186. $\beta$.

The Scad Tree.

In Hedges - not uncommon. April. S. 


\section{( 91$)$}

PTARMICA. R. S. 183 .

ACHILLEA Ptarmica. H. F. 325 .

Sneeze-wort, Baftard Pellitory, or Goofe-tongue. In Davington Brooks - uncommon. Auguft. P.

PULEGIUM. R.S. 235.

MENTHA Pulegium. H. F. 224.

Penny-royal, or Pudding-grafs.

In a fmall Pond at Elvyland in Ofpringe-uncominon.

PYRASTER feu Pyrus fylveftris. R.S. 452.

PYRUS communis. H. F. 189.

The Wild Pear Tree.

In the Wood leading from Keneways to Wilderton very uncommon. April. S.

\section{Q.}

QUERCUS latifolia. R.S. 440.

QUERCUS Robur. H. F. 359 .

The common Oak Tree.

InWoods-very common. April. P.

QUEERCUS latifolia mas quæ brevi pediculo eft. R. S. 440.

QUERCUS Robur. H. F. 359. $\beta$.

The Oak Tree.

In Woods ant Hedges-common. April. S. 


\section{$(92)$}

R.

RADICULA fylveftris feu paluftris. R. S. 301 . SISYMBRIUM ampbibium. H. F. 258. $\alpha$. Water Radith.

About the Powder-Mill Waters not common. June. $P$.

RADIOLA vulgaris ferpyllifolia Millegrana! minima. R. S. 345.

LINUM Radiola. H. F. I 7 .

The leaft Rupture-wort, or All-feed.

On Sandy Banks between Ore and Luddenham common. A.

RANUNCULO five Polyanthemo aquatili albo affine. R. S. 250.

RANUNCULUS aquatilis. H. F. $213 \cdot \gamma$.

Fennel-leaved Crow-foot.

In Running Waters - common. May. P.

RANUNCULUS aquaticus albus, circinatis tenuilfime divifis foliis, floribus ex alis longis pediculis innixis. R. S. 249.

RANUNCULUS aquatilis. H. F. $213 . \delta$.

Fineft leaved Water Crow-foot.

In the Brent Dykes $=$ common. April to June. P. 


\section{(93)}

$\mathrm{R}+\mathrm{NUNCULUS}$ aquatilis. R. S. 249.

RANUNCULUS cquatilis. H. F. 213 .

Various leaved Water Crow-foot.

In the fhooting Meadow Stream - common.

May. P.

RANUNCULUS aquatilis hederaceus albus.

R. S. 249.

RANUNCULUS bederaceus. H. F. 212 .

Ivy leaved Water Crow-foot.

By the Wall that lerids to Thorn-common. May. P.

RANUNCULUS aquatilis omnino tenuifolius.

R. S. 249.

RANUNCULUS aquatilis. H.F. $213 . \beta$.

Fine leaved Water Crow-foot.

In C fpringe River near Whitehill-common. April to June. P.

RANUNCULUS arvorum. R. S. 248.

RANUNCULUS arvenfis. H. F. 212 .

Corn Crow-foot.

In Corn fields-very common. June. A.

RANUNCULUS bulbofus. R. S. 247.

RANUNCULUS bulbofus. H. F. $21 \mathrm{I}$.

Round rooted or bulbous Crow-foot, or Butter-

cups

In Meadows - very common. May. P.

RANUN- 


\section{( 94$)$}

RANUNCULUS flammeus major. R. S. 250. RANUNCULUS Lingua. H. F. 210.

Great Spear-wort.

In the old Haven near Sand wich - very uncommon. May. P.

RANUNCULUS flammeus minor. R. S. 250. RANUNCULUS Flammula. H.F. 210. The leffer Spear-wort.

In the fhooting Meadow Stream-common. June to September. P.

RANUNCULUS nemorofus dulcis fecundus Tragi. R. S. 248.

RANUNCULUS Auricomus. H. F. 211 ?

Sweet wood Crow-foot, or Goldilocks.

In tVoöls and under Hedges-common. A pril. P.

RANUNCUL.US paluftris: R. S. 249.

RANUNCULUS sceleratus. H. F. 212.

Round leaved Water Crow-foot.

On the Banks of the Brent Marthes — common. June. A.

RANUNCULUS pratenfis erectus acris. R. S. 248.

RANUNCULUS acris. H. F. $21 \mathrm{r}$.

Upright Meadow Crow foot.

In Meadowes - very common. June. P.

RANUNCULUS pratenfis repens. R. S. $24 \%$.

RANUNCULUS repens. H. F. 210.

Common creeping Crow-foor.

On Beacon Hill - common. May. P. 


\section{$(95)$}

RAPA fativa rotunda. R. S. 294.

BRASSICA Rapa. H. F. 253.

Turneps.

In Corn fields, and by Way fides — not uncostmon. April. B.

RAPISTRUM arvorum. R. S. 295.

SINAPIS arrenfis. H. F. 260.

Charlock, or Wild Muftard.

In Fields among Corn-common. May. A.

RAPISTRUM fore luteo, filiqua glabra articulata.

R. S. 296 .

RHAPHANUS Rbapbaniftrum. H.F. 252. $\beta$.

Yellow flowered Charlock with a jointed Cod.

In Fields among Corn - common. July. A.

RAPUNCUL.US Scabiolæ capitulo cæruleo. R. S. 278.

JASIONE montana. H. F. 329.

Hairy Sheep's Scabious.

By the Way fides near Dunkirk - uncommon. Augurt. A.

RESEDA vulgaris. R. S. 366 .

RESEDA lutea. H. F. 18 I.

Bafe Rocket.

In Boughton Chalk Pits - not common. July. A. 


\section{$\left(9^{6}\right)$}

RHAMNCIDES fructifera, foliis Salicis, baccis leviter flavefcentibus. R. S. 445 .

HIPPOPHAE Rbamnoides. H. F. 368.

Sea Buck Thorn, or Sallow Thorn.

In Shepey, uncommon; near Sandown Caftle plentifully. April. S.

RHAMNUS catharticus. R. S. 466 .

RHAMNUS catbarticus. H. F. 83.

Buck Thorn, or Purging Thorn.

In Perry Wood and Badgen Down Hedges - not - common. May. S.

RHAPHANISTRUM filiqua articulata glabra majore et minore. R. S. 296.

RHAPHANUS Rbaphanifrum. H. F. 252.

White flowered Charlock with a jointed Cod.

In Fields among Corn - common. June. A.

RHAPHANUS rufticanus. R. S. 301 .

COCHLEARI. Amoracia. H. F. 278.

Horfe Radifh.

On the Banks of the River, abore the Sluice, and of Ore Stream-unconimon. June. P.

RIBES nigrum vulgo dictum fólio olente. R.S. $45^{6}$.

RIBES nigrum. H. F. 85 .

Black Currants, or Squinancy Berries.

In the boggy Ofier Ground near Stone Bridge - wncommon. May. S.

RIBES 
* RIBES vulgare fructu rubro. R. S. 465.

RIBES rubrum. H. F. 84.

Currants.

In Some tbick Hedges in Ofpringe - not common.

May. S.

ROSA fylveftris altera minor, flore albo noftras.

R. S. 455 .

ROSA arien/is. H. F. 192.

White flowered Dog's Rofe.

In Hedges - very common. June. S.

ROSA fylvertris inodora feu canina. R. S. $454^{\circ}$.

ROSA canina. H. F. 192.

Red flowered wild Dog's Rofe, or Hep Tree.

In Hedges - very common. June. S.

* ROSA fylveftris odora. R. S. 454.

ROSA Eglanteria. H. F. $19 \mathrm{I}$.

Sweet Briar.

In a Fiedge near Ore.Stray — very uncommon. June. $S$.

ROSA pumila fpinofifima, foliis Pimpinellæ glabris flore albo. R. S. 455 . ROSA Jpinofifima. H. F. IgI. The Burnet Rofe.

On the North fide of Chatham Hill _- uncommon. June, $S$.

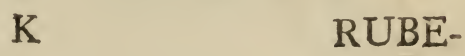




\section{$\left(9^{8}\right)$}

RUBEOLA arvenfis repens cærulea. R. S. 225. SHERARDIA arvenfis. H. F. 54.

Little Field Madder.

In Cades nerw Wood, and in Broom fields in Hernhill - not uncommon. May. A.

RUBEOLA vulgaris quadrifolia lævis floribus purpurafcentibus. R. S. 225.

ASPERULA Cynancbica. H. F. 55 .

Squinancy Wort.

In Ofpringe Cbalk Pits, and on Badgen Down July. P.

RUBUS Idæus fpinorus fructu rubro. R. S. $4^{6} 7$. RUBUS Ideus. H.F. 193.

The Rafpberry Burh.

In Stocking Wood, near Lees Court, and in Perry Wood - not common. May. S.

RUBUS major fructu nigro. R.S. 46\%.

RUBUS fruticofus. H.F. 193.

The Bramble, or Blackberry Bufh.

In Woods and Hedges - very cummon. June. S.

RUBUS minor fructu cæruleo. R. S. 467 .

RUBUS cafus. H. F. 193.

The fmall Bramble, or Dew-berry Bufh. In Woods and Hedges - common. July. S. 


\section{( 99 )}

RUSCUS. R. S. 262.

RUSCUS aculeatus. H.F. 373.

Knee Holly, or Butcher's broom.

In Ofpringe Wools and Hedges-common: April. P.

RUTA muraria. R. S. 122.

ASPLENIUM Ruta muraria: H. F. 386.

Wall Rue, White Maiden-hair, Tentwort.

On the Buttrices near the South Door of the Church very uncommon. $P$.

S.

SALICARIA vulgaris purpurea foliis oblongis,

R. S. 367 .

LYTHRUM Salicaria: H. F. 179.

Purple-fpiked Loofeftrife, or Willow. herb.

In the fwampy Ground near Stone-bridge - not ws-

common: Auguft. P.

SALICORNIA geniculata annua. R. S. 136 .

SALICORNIA europaa. H. F. I.

Marih Sampire, Jointed Glafs-wort, or Salt-wort. In the Marbes as you go to Thorn - common. Auguft. A.

SALIX. R. S. $447^{\circ}$

SALIX alba. H.F. 366.

The common white Willow.

In moift Places about the Powder Mills-not common. April. S.

SALIX 


\section{$(100)$}

SALIX folio articulato fplendente flexilis. R. S. 448.

SALIX amygdalina. H. F. ${ }_{36} 6$.

The rount eared Mining Willow, or Almondleaved Willow.

In a Hedge as you enter Sheldwich Road-uncommon. May. S.

SALIX folio longiffimo. . R. S. 450 .

SALIX vimenalis. H. F. ${ }_{366 .}$

The Ofier.

In moift Places - common. April. S.

SALIX latifolia rotunda. R. S. 449.

SALIX caprea. H. F. 366 .

The common round-leaved Sallow.

In Woods and Hedges - common. A pril. S.

SALIX pumila foliis utrinque candicantibus et

lanuginofis. R. S. 447 .

SALIX arenaria. H. F, 364 .

Sand Willow.

On the Sand Downs near Deal-plentifully. June. S.

SAMBUCUS. R. S. 461 ,

SAMBUCUS nigra. H. F. 112.

Common Elder.

In Hedges - very common. May. S. 


\section{$(101)$}

SAMBUCUS humilis, feu Ebulus. R. S. 461. SAMBUCUS Ebulus. H. F. 113 .

Dwarf Elder, Wall-wort, or Dane-wort.

By the Road fides near Boughton-Street — not uncommon. July. P.

SAMOLUS Valerandi. R. S. 283.

SAMOLUS Valerandi. H. F. 79.

Round.leaved Water Pimpernel.

In the Brent Marfhes - common. June. P.

SANGUISORBA minor R. S. 203.

POTERIUM Sanguiforba. H. F. $35^{8}$.

Burnet.

On the chalky Hills in Ofpringe-common. July. P.

SANICULA five Diapenfia. R. S. 221.

SANICULA europaa. H. F. 96.

Sanicle. .

In.Woods - very common. May. P.

SAXIFRAGA aurea. R. S. 158.

CHRYSOSPLENIUM oppofitifolium. H. F. $1 ; 6$.

Golden Saxifrage.

In Jud's Wood - not common. April. P.

$$
\mathrm{K}_{3}
$$




\section{$(102)$}

SAXIFRAGA rotundifolia alba. R. S. 354 .

SAX!FRAGA gramulata.' H. F. 159.

White Saxifrage, or Sen-green.

Upon Beacon Hill - uncommon. May. P.

SAXIFRAGA verna annua. R. S. 354.

SAXIFRAGA tridaciylites. H. F. 159.

Rue-leaved Saxifrage, or Whitlow-grafs.

On Houfes andWalls - very common. A pril. A.

SCABIOSA major communior folio laciniato.

R. S. Igl.

SCABIOSA arvenfis. H. F. 50 .

Common Field Scabious.

In moift Fields among Corn, and on Bank fides-common. Augutt. P. With a proliferous Flower in Badgen Wood, uncommon.

SCABIOSA minor vulgaris. R. S. Ig $\mathrm{r}$.

SCABIOSA Columbaria. H. F. $5^{\mathrm{C}}$.

The leffer Field Scabious.

In dry billy Places - not uncommon. July. $\mathrm{P}$.

SCABIOSA radice fuccifa, fore globofo. R.S. 19 !. SC $\triangle$ BIOSA Succisa. H. F. 50 .

Devil's Bit.

In Dyfing Wood and the Abby Meadows-common. Aliguit. $P$.

SCAN: 


\section{( 103 )}

SCANDIX femine roft tato vulgaris. R. S. 207.

SCANDIX Pecten Veneris: H. F. 107.

Shepherd's Needle, or Venus Comb.

In Fields among Corn-very common. July. A.

SCORO ONIA feu Salvia agreftis. R. S. 245 .

TEUCRIUM. Scorodonia. H. F. 219.

Wood Sage.

In Byfing Wood - very common. July. P.

SCROPHULARIA aquatica major. R. S. ${ }^{*} 283$.

SCROPHULARIA aquatica. H. F. 239.

Water Betony, Water Figwort.

On the Banks of the River, and in moift Ditchescommon. July. P.

SCROPHULARIA major. R. S. * 283.

SCROPHULARIA nodofa. H. F. 239.

Common knobby-rooted Figwort. In moift Hedges - common. July. P.

SEDUM minimum non acre flore albo. R.S.

270.

SEDUM annum. H. F. 172.

Mountain Stone-crop.

On the Bcacb near Sanduwn Cartle - plentifully. Auguit. P. 


\section{(104)}

SEDUM minus hæmatoides. R. S. $26 \mathrm{~g}$.

SEDUM refiexum. H. F. 1;0. $\beta$.

Yellow Stone-crop, or Prick-madam.

On the Abby Walls - common. July. P.

SEDUM minus luteum ramulis reflexis. R. S. 270.

SEDUM reflexum. H. F. I70.

Yellow reflexed Stone-crop.

On the Abby Walls - common. July. P.

SEDUM parvum acre flore luteo. R. S. 270.

SEDUM acre. H. F. I 7 r.

Stone-crop, or Wall-pepper.

On Walls and Houfes - not uncommon. July. P.

SEDUM fexangulare. H. F. I7I.

Infipid Stone-crop.

Near Sheernefs plentifully. July. B.

SEMPERVIVUM majus. R.S. 269.

SEMPERVIVUM teEtorum. H. F. 185.

Houfe-leek, or Sengreen.

On Walls and tiling of Houfes not uncommon.

July. P.

SENECIO 


\section{( 105$)$}

SENECIO vulgaris. R. S. 178.

SENECIO vulgaris. H. F. 315 .

Common Groundfel, or Simfon.

In Fields and under Hedges - very common. May. A.

SERPYLLUM citratum. R. S. 230.

THYMUS Serpyllum. H. F. 229. $\varepsilon$.

Lemon Thyme.

On Badgen Down - not common. July. P.

SERPYLLUM vulgare. R. S. 230.

THYMUS Serpyllum. H. F. 229.

Mother of Thyme.

On dry billy Ground - very common. July. P.

SERRATULA. R. S. 196.

SERRATULA tincioria. H. F. 304.

Saw-wort.

Near the late Decoy Ponds at Graveney-uncommon.

July. P.

SESELI pratenfe noftras. R. S. 216.

SESELI Caruifolia. H. F. 106.

Meadow Saxifrage.

In the low Meadows in Hernhill, and in Shepey not uncommon. Auguft. P. 


\section{$(106)$}

SIDERITIS anglica ftrumofa radice. R. S. 242 . STACHYS paluftris. H. F. 227.

Clowns Allheal.

In moift Places, and near the River - common. Auguit. $\mathrm{P}$.

SIDERITIS arvenfis rubra. R. S. 242.

GALEOPSIS Ladanum. H. F. 225.

Narrow leaved Allheal, or Iron-wort.

In Ofpringe Corn fields - common. Augurt. A.

SIDERITIS humilis lato obtufo folio. R. S. 242.

GLECHOMA arvenfis. H. F. 224.

Upright Ground Ivy.

In Ore Corn fields - common. Auguft. A.

* SINAPI album filiqua hirfuta, femine albo vel ruffo. R. S. 295.

SINAPIS albe. H. F. 260.

White Muttard.

By the Way fide in the Brent Marh-very uncommon. Auguft. A.

SINAPI fativum fecundum. R. S. 295:

SINAPIS nigra. H. F. 260.

Common Multard.

In Shepey amongft Corn, and by Wayfides - plentifully. July. A. 


\section{( 107 )}

SISYMBRIUM Cardamine feu Nafturtium aquaticum. R. S. 300.

SISYMBRIUM Nafturtium.

Water Crefs.

By the River fides - very common. July. B. or P.

SISYMBRIUM hirfutum. R. S. 233.

MENTHA aquatica. H. F. 223. $\mathrm{\gamma}$.

Water Mint.

On the fides of the River - very common. July. P.

SIUM aromaticum. Sifon officinarum. R. S. 211. SISON Amomum. H. F. 104.

Baftard Stone Parnley.

In a Hedge near Thorn - not uncommon. Auguft. P.

SIUM arvenfe five fegetum. R.S. 211 .

SISON fegetum. H. F. 104.

Corn Parney, or Honewort.

In moift Hedges near Ewell - not uncominon. July. B.

SIUM latifolium foliis variis. R. S. 2 II.

SIUM latifolium. H. F. 103.

Great Water Parfnep.

In marhy Ground, and by the River fide common. Auguit. P. 


\section{( 108$)$}

SIUM pufillum foliis variis. R. S. 212 .

SISON inundatum. H. F. 104 .

The leaft Water Parfnep.

In the Dykes of Nagden MarR — not common.

June. A.

SIUM umbellatum repens. R. S. 2 Ir.

SIUM nodiforum. H. F. 103.

Creeping Water Parfnep:

In the Abby Ditches - common. Auguit. P.

SMYRNIUM. R.S. 208.

SMYRNIUM Olufatrum. H. F. 109 .

Alexanders.

In a Hedge as you enter Graveney Marh-uncommon.

June. B.

SOLANUM lignofum feu Dulcamara. R.S. 255. SOLANUM Dulcamara. H. F. 78.

Woody Night-fhade, or Bitter-fweet.

In moift Woods and Hedges - common. July. P.

* SOLANUM pomo fpinofo oblongo flore calathoide, Stramonium vulgo dictum. R.S. 266. DATURA Stramonium.. H. F. 78 .

Thorn Apple.

On Dunghills - very uncommon. Augutt. A. 


\section{$(\log )$}

SOLANUM vulgare. R. S. 265 . SOLANUM nigrum. H, F. 78 .

Common Night-hade. On Rubbijh and Dungbills - very common. Ju'y. A. SONCHUS afper laciniatus. R. S. $16_{3}$. SONCHUS oleraceus. H. F, $294 . \%$ Prickly Sow-thiftle with jagged Leaves. On Rubbifs and in cultivated Grounds _... common. Auguit: A.

SONCHUS afper non laciniatus. R. S. 163. SONCHUS oleraceus. H. F. 294. $\delta$. Prickly Sow-thifle with lefs jagged Leaves. In cultivated Grounds, and on Rubbifb- common. June to Auguif. A.

SONCHUS lævis. R.S. $16_{2}$. SONCHUS oleraceus. H.F. 294. $\alpha_{0}$ Common Sow-thiftle.

On Rubbijh, in Fields and cultivated Grounds - very common. June to September. A.

SONCHUS Jævis minor paucioribus laciniis. R. S. $16_{3}$.

SONCHUS oleraceus. H. F. 294. $\beta$. Smooth Sow-thiftle with lefs jagged Leares. On Rubbijh, in Fields and c itivated Grounds. June to September. A. 


\section{( 110$)$}

SONCHUS repens, multis Hieracium majus.

R.S. 163 .

SONCHUS arrenfis. H. F. 295.

Tree Sow-thiftle.

In Fields and Hedges - commorr. Auguft. P.

SORBUS fylveftris foliis domefticæ fimilis.

R.S. 452 .

SORBUS aucuparia. H. F. 189 .

Mountain Af, Quicken Tree, Roan Tree.

In tle Blean Woods - not uncommon. May. S.

SPARGANIUM non ramofum. R. S. 437.

SPARGANIUM eręlum. H. F. 346. $\beta$.

Bur-reed not branched.

In the Ditckes of Ham and Graveney Marhes common. July. P.

SPARGANIUM ramofum. R. S. 437.

SPARGANIUM erectum. H. F. 346.

Great branched Bur-reed.

In Ponas near Ewell - not uncommon. July. P.

SPERGULA maritima fore parvo cæruleo, femine vario. R.S. 35 s.

ARENARIA marina. H. F. 169. $\gamma_{0}$ ?

Small flowered Sea-fpurrey.

In Shepey Inand - not uncommon. July. P. SPER- 


\section{( III)}

SPERGULA purpurea. R. S. 163. ARENARIA mbra. H. F. I $6 \mathrm{~g}$.

Purple Spurrey, Purple fiowered Chickweed. On fandy Grounds in Hernhill _- not uncommon. July. A.

SPHONDYLIUM. R. S. 205.

HERACLEUM Spbondylium. H. F. 102.

Cow Parinep.

On the fides of Fields, and in Heages $\longrightarrow$ common: July. B.

STATICE montana minor. R. S. 203.

STA TICE Armeria. H. F. 114.

Thrift, or Sea Gilly-Flower.

In cll the Salt Marhes- very common. July. P.

STELLARIA. R. S. $28 \mathrm{~g}$.

CALLITRICHE verna. H. F. 2.

Vernal Star wort, Water wort, Star-headed

Chickweed.

In the Powder Mill Streams-common. April. A.

STELLARIA aquatica follis longis tenuifrimis.

R. S. 290 .

C.ALLITRICHE autumnalis. H. F. 2.

Autumnal Star-wort.

In the River oppofite the Gunpowder Stove - no. uncommon. September. A.

t. 2 .

STRA- 


\section{$($ II $)$}

SRRATIOTES foliis Afari, femine rotundo. R. S. 290.

HYDROCHARIS Morfus rana. H. F. 372 . Frog-bit.

In the Dykes of Ham and Graveney Marnhes common. June. P.

SYMPHYTUM magnum. R. S. 230. SYMPHYTUM offinale. H. F. 68. Comfrey.

On the Banks of the Powder Mill Waters-uncommon. July. P.

\section{T.}

' ANNUS racemola, flore minore luteo palle. fcente. R.S. 26?. TAMUS communis. H. F. 369 . Black Briony.

Uncer Hedges, and among Bufkes-crnmon. June. P. TANACETUM. R. S. 188. TANACETUM vulgare. H. F. 310.

Common Tanfy.

By Way fides, wear Queen Court and Uplees - very uncommon. Auguft. P.

TAXUS. R. S. 445.

TAXUS baccata. H. F. 372.

The Yew Tree.

In Wcods and Hedges on a cbalky Soil _- common. April. S. 


\section{( $\operatorname{Ir} 3)$}

THALICTRUM feu Thalietrum majus. $R$. $S$. 203.

THALICTRUM flarum. H. F. 216.

Meadow Rue.

In the fhooting Meadow Stream - uncommon. June. $P$.

TITHYMALUS characias Amygdaloides. R. S, 312.

EUPHORBIA Amygdaloides. H. F. I84.

Wood Spurge.

In Woods, and under Hedges in Ofpringe - very common. April. P.

TITHYMALUS heliofcopius. R. S. 3 I 3 .

EUPHORBIA Helioscopia. H. F. 183 .

Sun Spunge, or Wart-wort.

In Meadoress, and on Dungbills_common. July. A:

TITHYMALUS leptophyllos. R. S. $3^{1} \mathbf{3}$.

EUPHORBIA exigua. H.F. 182.

Dwarf Spurge, Small annual Spurge.

In Corn fields and Gardens - common. July. A.

TITHYMALUS parvus annuus, foliis fubrotundis

non crenatis, Peplus dictus. R. S. 3 '3.

EUPHORBIA Pep'us. H. F. 182.

Petty Spurge.

In cultivated Grounds - common. July. A.

$$
\text { L } 3
$$

TITHY:- 


\section{( 114$)$}

TITHYMALUS platyphyllos Fuchsii. R. S.

\section{2.}

EUPHORBIA platyphyllos. H. F. I 84.

Broad leaved Spurge.

In Corn fields near Thorn Creek common.

$$
\text { July. P. }
$$

TORMENTILLA. R. S. 257.

TORMENTILLA ereeza. H. F. 197.

Tormentil Septfoil.

In Woods and Hedges - conmon. June. P.

TORMENTILLA reptans. R. S. 257.

TORMENTILLA reptans. H. F. 198.

Creeping Tormentil.

In Brcom fields at Hernhill - common. July. P.

TRAGOPOGON luteum. R. S. I7I. TRAGOPOGON pratense. H. H. 293. Yellow Goat's Beard, Go to Bed at Noon. In Fields and Paftures - common. June. B.

TRAGOPOGON purpureum. R.S. 172.

TRAGOPOGON porrifolium. H. F. 293.

Purple Goat's Beard.

In a Marfs near Sheernefs = uncommon. June. $B$. 


\section{( 115$)$}

TRICHOMANES. R.S. IIg.

ASPLENIUM Tricbomanes. H. F. 385 .

Englifh black Maiden-Hair.

On the Walls of the Church and Abby- not comm mon. $\mathrm{P}$.

TRIFOLIUM arvenfe humile fpicatum feu

Lagopus. R. S. 330.

TRIFOLIUM arvense. H. F. 285.

Hare's Foot, or Hare's Foot Trefoil.

Upon Beacon Hill - not uncommon. July. A.

TRIFOLIUM cochleatum folio cordato maculato.

R.S. 333 .

MEDICAGO arabica. H. F. 288.

Heart Trefoil, or Clover.

In the Abby Meadows-not uncommon. June. A.

TRIFOLIUM cochleatum modiolis fpinô̂s.

R. S. 333 .

MEDICAGO arabica. H. F. 288. $\gamma$.

Hedge-hog Trefoil, or Clover.

In Paftures near Sheernefs-not common. May. A.

TRIFOLIUM fragiferum. R. S. 329 .

TRIFOLIUM fragiferum. H. F. 286.

Strawberry Trefoil.

In the Abby Meadows - common. Auguft. P:

TRIFO: 


\section{$(116)$}

TRIFOLIUM flofculis albis, in glomerulis oblongis afperis, cauliculis proxime adnatis. R. S. 329 .

TRIFOLIUM scabrum. H. F. 285.

Oval headed Trefoil.

in Paftures in Shepey - common. June. A.

TRIFOLIUM lupulinum alterum minus. R.S. 330.

TRIFOLIUM procumbens. H. F. 287.

The leffer Hop Trefoil, Decumbent Trefoil.

In Meadows - common. May to Auguft. P.

TRIFOLIUM lupulinum minimum. R. S. 33 r. TRIFOLIUM fliforme. H.F. 287.

Small Trefoil.

On gravelly Banks beyond South Street - uncommon.

May. A.

TRIFOLIUM pratenfe album. R. S. 327.

TRIFOLIUM repens. H. F. 283 .

Dutch Clover, White flowered Meadow Trefoil.

In Fields anả Meadows - very common. May to

September. P.

TRIFOLIUM pratenfe luteum capitulo L Lupuli, vel. agrarium. R. S. 330.

TRIFOLIUM agrarium. H. F. 286.

Hop Trefoil.

On Beacon Hill - not uncommon. June. A. 


\section{( 117 )}

TRIFOLIUM pratenfe purpureum. R.S. $3 z 8$. TRIFOLIUM pratenfe. H. F. 284.

Common Purple or Honey-fuckle Trefoil.

In Fields and Meadows - very common. May to - September. P.

TRIFOLIUM purpureum majus, folis longioribus et anguftioribus, floribus faturatioribus. R. S. 328 .

TRIFOLIUM medium. H. F. 284.

Long leaved Purpie Trefoil with deeper coloured Flowers.

In Byfing Wood, and by Field fides in Ofpringe not uncommon. July. P.

TRIFOLIUM ftellatum glabrum. R. S. 329 :

TRIFOLIUM maritimum. H. F. 284.

Teafel-headed Trefoil.

On Shellnefs in Shepy - uncommon. June. P.

TURRITIS muralis minor. R. S. 294.

TURRITIS birfuta. H. F. 254 .

Hairy Tower Muftard.

On old Walls at Colkins in Boughton-very uncunn mon. May. B.

TUSSILAGO. R. S. 173 .

TUSSILAGO Farfara. H. F. 315 .

Common Colt's Foot.

In moift Fields - very common. A pril. P.

TYPHA. 


\section{$(118)$}

TYPHA. RS. 436 .

TYPHA latifolia. H. F. 345.

Great Cat's 'Tail, or Reed Mace.

On the fides of Ponds, and in Dykes - very common. July. P.

TYPHA paluftris media. R. S. 436 .

TYPHA angufifolia. H. F. 345 .

Narrow leaved Cat's 'Tail.

In the Ditches at Ewell and Nagden-not uncommion. July. P.

\section{V.}

VALERIANA fylveitris major. R. S. 200 ,

VALERIANA offinalis. H. F. 12.

Great wild Valerian.

On the Banks of Ofpringe River - not uncommon. June. $P$.

VALERIANA fylveftris minor. R. S. 200.

VALERIANA dioica. H.F. I 2.

Small wild or Marih Valerian.

On the Banks of the Thooting Meadow Stream common. June. P.

VALERIANELLA arvenfis præcox humilis femine compreffo, five Lactuca agnina. R. S. 201.

VALERIANA Locufa. H. F. I 2.

Lamb's Lettuce, or Corn Sallad.

In Corn fields, and rich cultivated Ground - common.

May. A. VALE- 


\section{(-19)}

VALERIANELL\# vulgaris fpecies major, ferotina. R. S. $20 \mathrm{I}$.

VALERIANA Locufa. H. F. 13. $\beta$.

Late flowering Lambs Lettuce, or Corn Sallad.

In the Corn fields between Ore ond Harty-ferry - not common. July. A.

VERBASCUM flore albo parvo. R.S. 287. VERBASCUM Lycbritis. H. F. 76 .

Hoary Mullein, or white Mullein.

On old Walls at Davington-not common. July. B. 1

VERBASCUM mas latifolium luteum. R.S. 287. VERBASCUM Thapfus. H. F. 75 .

Great white Mullein, High Taper, Cows Lungwort.

On dry Banks by Road fides - common. July. B.

VERBASCUM nigrum flore parvo, apicibus pur: pureis. R. S. 288.

VERBASCUM nigrum. H. F. 76 .

Sage-leaved black Mullein.

On the fides of Cbalk felds in OPpringe - very uncomsmon. As you afcend St. Martin's-Hill near Canterbury - plentifully. July. P.

VERBENA vulgaris. R. S. 236 .

VERBENA officinalis. H. F. 505 .

Vervain.

By Road fides and Foot patbs-very common. July. P.

VERBE. 


\section{$(120)$}

VERBESINA feu Cannabina aquatica fore minus pulchro elatior. R.S. $18 \%$. BIDENS tripartita. H. F. 309 .

Trifid Water-hemp Agrimony.

In marfoy watery Places - common. July. A.

VERBESINA pulchriore flore luteo. R. S. 187.

BIDENS cernua. H. F. 309.

Whole leaved Water-hemp Agrimony.

In Dykes near the Town Key - common. Augurt. A.

VERONICA aquatica anguftifolia minor. R.S. 280.

VERONICA fcutellata. H.F. 5 .

Narrow leaved Water Speedwell, or Brooklime. In wet Ditcbes and mar by Ground-common. June. P.

VERONICA aquatica longifolia media. R.S. 280. VERONICA Anagallis. H. F. 5 .

The middle long leaved Water Speedwell, or Brooklime.

In the Abby Ditches - common. July. P.

VERONICA aquatica rotundifolia Becabunga dieta minor. R. S. 280.

VERONICA Becabunga. H. F. 4.

Common Brooklime.

In wet Ditches and fmall running Waters — very common. May. P. 


\section{$($ I2I $)$}

VERONICA Chamædrys fylveftris dieta. R.S. 28 : VERONICA Chamedrys. H. F. 5 .

Wild Germander.

In Fields and Meadcws - very common. May. P.

VERONICA floribus fingularibus, in oblongis pediculis, Chamædryfolia. R. S. 279.

VERONICA agrefis. H. F. 6.

Germander Speedwell, or Chickweed.

In Fie'd, on Rubbish, and in cultivated Ground

conmon. May. A.

VERONICA flofculis fingularibusHederule folio,

Morfus gallinæ minor dicta. R. S. 280.

VERONICA bederifolia. H. F. 6.

lvy leaved Speedwell, or fmall Henbit.

In diry Fieids, and on old Walls-common. A pril. A.

VERONICA mas fupina et vulgatifima. R. S.

. $28 \mathrm{I}$.

VERONICA oficinalis. H.F. 4 .

The Male Speedweil, or Fluellin.

In barren dry Fields - common. May. P.

VERONICA pretenfis minor ; five BetonicaPauli.

R.S. 279.

VERONICA Serpyliffolia. H. F. 4 .

Little, or fmooth Speedwell, or Paul's Retony. In Fields and Meadows - common. May. P. 
VIBURNUM. R. S. 460.

\section{VIBURNUM Lantana. H. F. 112.}

The pliant Mealy, or Way-faring Tree. In Hedges - common. May. S.

VICIA. R. S. 320 .

VICIA faiva. H. F. 278.

Common Vetch, or Tare.

In Fields among Corn - not uncominon. June. A.

VICIA fepium perennis. R. S. 320 .

VICIA Sepium. H. F. $27^{8}$.

Bun Vetch.

In bufay Places and Hedges-common. May. P.

VICIA minima præcox Parifienfium. R. S. 321.

E.R VUM Jolsniense. H. F. 279.

Spring Tare.

in Ofpringe Cbalk Pits - common. April. P.

VICIA fylveitris five Cracea major. R. S. 321.

VICIA lathyroides. H. F. 279.

Strangle Tare, or Wild Vetch.

In fondy Fields in Hernhill — not uncomainon. May. A. 


\section{( 123$)$}

VINCA Pervinca minor. R. S. 268.

VINCA minor. H.F. 77 .

Periwinkle.

In Cades Wood, and Hedges in Prefton and Orpringe - common April. P. Wirh a white Flower, near the 3.9 th Mile Stone - very uncommon.

VIOLA bicolor arvenfis. R. S. 366 .

\section{VIOLA tricolor. H. F. 33I. $\beta$.}

Panfies, Heart's-eafe, Two Faces under a Hood. In fandy Corn fields at Hernhill _ common. Auguit. A.

VIOLA martia inodora fylveftris. R. S. $3^{6} 4$.

\section{VIOLA canina. H.F. 33 r.}

Wild or Dogs Violet. In Woods and under Hedges-very commoir. A pril. L'.

VIOLA martia hiriuta inodora. R. S. $3^{6} 5$.

VIOLA birta. H. . 330 .

Hairy Violet.

In the new Wood at Cades in Ofpringe - plen if alls. March. P.

VIOL.A martia alba. R. S. ${ }_{364}$. VIOLA odorata. H. F. $330 . \beta$. White Violet.

Under dry Hedges and Banks, by Way fides - not uncommon. March, P. 
VIOLA martia purpurea. R. S. 364 .

V!OL.A odorata. H. F. 330.

Purple fweet Violet.

Uncier Hiedges, and in Fields - very common. March. P.

VIOLA tricolor. R. S. $36 r$.

VIOLA tricolor. H. F. 33 I.

Panfies, Three liaces under a Hood.

In Corn fields at Ore, and in Gordens - common. June. A.

VIRGA aurea. R. S. 176 .

SOLIDAGO Virga ourea. H.F. 318.

Common Golden Rod.

I.t Wrous ard Hedges-common. Augurt. P.

VISCUM. R. S. 464 .

VISCUM album. H. F. 367 .

Mifs, or Miffeltoe.

On Trees, especially in Orchards common. May. S.

VUILNERARIA ruftica. R. S. 325 . ANTHYLLIS Vulneraria. H. F. 273.

Kidney Vetch, or Ladies Finger.

About Byfing Wood common. July. P.

ULMA 


\section{$(125)$}

U.

ULMARIA. R. S. 259.

SPIR ÆA Uimaria. H. F. 19 .

Meadow fweet.

In moift Meadorvs in Davington-common. July. P.

ULMUS folio glabro.

ULMUS glabra. H. F. 95. $\beta$.

The fmooth leaved or Wych Elm. In Hedges-not uncommon. April. S.

ULMUS folio latifimo fcabro. R. S. 469 .

ULMUS glabra. H. F. 95 .

The Wych Hazel, or broad-leaved Elm.

In Heiges at Throwleigh - uncommon. April. S.

ULMUS vulgatifima, folio lato fcabro. R.S, 468.

ULMUS campeftris. H. F. 94 .

The common Elm.

In Hedges - very common. April. S.

URTICA minor. R. S. 140.

URTICA urens. H. F. 355 .

The leffer Stinging Nettle.

In cultivated and uncultivated Ground - very common. Auguft. A. 


\section{( 126$)$}

URTICA racemifera major perennis. R. S: 139. URTICA dioica. H. F. 355 .

The common Stinging Nettle.

Under Hedges, and on Rubbis — very common July. $P$.

\section{P L A N T S OMIT TE D.}

* CALENDULA officinalis. Lin. Syft. Nat. II.

$$
\text { p. } 577 \text {. }
$$

Garden Marigold.

On the Beach at St. Margaret's at Cliff; plentifully. And in a Field at Woodnerborow - very uncommon. June. A.

CRAMBE maritima brafficæ folio. R. S. 307 .

CRAMBE maritima. H, F. 260.

Sea Colewort.

At St. Margaret's ct Cliff-uncommon. June. P.

HYDROCERATOPHYLLON folio afpero quatuor cornibus armato R. S. 135.

CERATOPHYLLUM demerjum. H.F. 35; Horned Pond-weed.

In Marja Dykes - Common. June. P.

* myagrltm. R. S. 302.

MYAGRUn fativum. H. F. 242.

Gold of Plcaiure.

Near Sandwich, among the Flax - plentifully.

June. A. TRI- 


\section{(127)}

TRIFOLLIUM luteum lupulinum. R. S. 33 ז. MEDICAGO lupulina. H. F. 288.

Melilot Trefoil.

In Patbs near Weftwood - not common. May to Augurt. A.

CAUCULIS nodofa echinato femine. R.S. 220. TORDILIUM nodofum. H. F. 98 .

Knotted Parley.

By the Road fide at Minfter in Shepey-not common May. A.

LINARIA antirrhinum dicta. R. S. 283. ANTIRRHINUM minus. H. F. 238.

The leaft Toad Flax.

In a Corn field near Whitehill, Selling -uncommon. A.

SENECIO minor latiore folio, five montana.

R. S. $17^{8}$.

SENECIO Jyluaticus. H. F. $3 \mathbf{1} 6$.

Mountain Groundfel.

In Badgen Wood-not common. Auguft. A.

OENANTHE aquatica triflora. R. S. 220 : OENANTHE fifulofa. H. F. 104. $\beta$.

The leffer Water Dropwort.

In Ditches at Graveney - common. July. P. 
FOSSILIA SHEPEIANA.

A

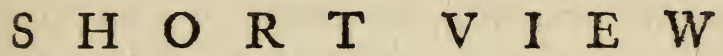

OF T H E

FOS I L B O D I E S

Native and Extraneous,

O F T H E

I $S \quad L \quad A \quad N \quad D$

O F

S H E P E $Y$,

IN T H E

C O U N T Y OF K EN T.

2uicquid fub terra eft in apricum proferet atas. 


\section{$(130)$}

\section{N T R O D U C T I O N.}

THE Ifland of Shepey, with the annexed Penin-

1 fulas of Harty and Elmly, furrounded by the Waters of the Thames, the Medway, and the Sea, is upwards of Thirty Miles in Circumference, about Tbirteeen in Length from Shellnefs to Sheernefs, and in its greateft Breadth about Six. It bath long been noted for producing large Quantities of Sbeep (from whence probably it derived its Name) as well as Corn; and exbibits to the inquifitive Naturalift a moft defirable Spot, by affording on its Surface many rare Plants, and more especially in the Bowels of its Northern Cliffs, So great a 2uantity and Variety of Foffils, both native and extraneous, as are Jcarcely to be paralled.

These Cliffs are in Length about Six Miles from Eaft to Weft, gradually declining at each End; the more elevated Parts whereof reach about two-tbirds of their extenfion, and are, at the very bigheft of them about Minfter, not lefs than Tbirty Yards in perpendicular Height abore the Beach or Sbore, and being composed of Clay, and conftantly waßhed at their Bafis by the 


\section{N T R O D U C T I O N.}

Tides wbich beat againft them more efpecially when driven by frong Eajterly Winds, they are continually weafting more or lefs, and falling down upon the Sbore, where the Clay being by degrees waßsed away, the Fofinls are left in great Abundance. So great is the Lojs of the Land at the higheit Parts, that fometimes near an Acre of it batb Junk down at once, though the fe Falls are indeed extraordinary.

Minfter, Shurland, and Warden, are the three. Manors to wbich theje Cliffs appertain, the Proprietors whereof bire them out to different Owners of Coperas Works, who employ the neigbbouring Poor to colle the Pyrites or Copperas Stone upon the Beach, at the Rate of about a Shilling per Bufbel, which they depojit in Heaps, when collecied, until a Quantity jufficient to load a Hoy is procured, when it is meajured and paid for.

These Coperas Gaiberers, from the Autbor's firft Vifits were inluced to prejerve and carry bome every Thing that feemed to their Apprebenfion fomewbat particular, and baving fince met with Encouragement, and acquired fome Information relative to what was moft defiruble by the Curious, they bave generally from that Time referved fuch, until Inquiries bove been made for them. Many rare Specimens, botb native and extrancous, far beyond any equal space of Ground, perbaps 


\section{I NTRONUCTION.}

perbaps the Universe can produce, are by these theans preferied. Indeed, more may tbus be obtained, at a very eafy Expence, than by the moft dilizent Fclfilifts alone, as they cannot allow due Time to examine the Shore fuficiently. This is evidently confirmed by comparing the Specimens from this Coaft, recited in the Works of our moft celebrated * Naturalifts, with those in the enfuing Catalorme. The Autbor doubts not that many curious Specimens bave efcaped bis Inquiries, and as the Cliffs are daily dropping their Contents more or lefs, be is fully affured that there will always remain a Variety fufficient to gratify every Lover of the Study, wbenever they may choose to take a Trip thitber. But alas! One difagreeable Circumftance attending a confiderabie Part of the Foffirs bere collected, is, that they are fo much impregnated with Pyritical Matter, that after being for fome Time p'aced in a Cabinet, the Salts thereof fboot and entirely deftroy them. Happy would it be, could fome cirtain Remedy be difcovered sobereby this Accident might be prevented. The Lofs of many valuable Specimens by this Couse, togetber witb bis Diftance from any inquifitive and abie Naturalifts, at laft induced the Autbor to difpose of bis wbole Collection to INGHAM FOSTER, E $\int_{\Omega}$;

- Dr. Grew, Mr. Llhuyd, Mr. Petiver, Dr. Woodward, Mr. Dale, and Dr. Hill. 


\section{$1 \mathrm{NT}$ T O DUCTION.}

Merchant of London, where it ncw forms no inconfiderable Part of that Gentleman's very valuable Cabinet.

He, many Years fince, entertained Tboughts of publifing a defcriptive Catalogue of all the Follits there, by bim collecied, but was prevented, as well by more neceffary Attarbments as the Difficulty of the Undertaking: However, it loving been intimated to bin that a concife Account of them would not bs demed an unacceptable Appendix to bis PLANT FE FAVERSHAMIENSES, in which Several of the Shepey Plants are recited, as no fpecial Account of thefe Folfils bath been offered to the Public, and as thofe that lave been alreacy noted by the before cited Autbors are difpersed in their WTorks, and as many others bave not as yet been publißsed, he complied evith the Propofal, and fet cobut revifing lis Notes and Objervations, and now offers thi, fhort Account of the Difcoveries made there in bis cmnual Vifits for above thefe Thirty Years paft; at firft folely for the Purpose of collecting the e elegant Bodies, and afterwards to Juperintend bis private Affairs, baving acquired fome Property in the 1/and, not far diftant from the Cliffs.

What the candid Reader may expect in the following Catalogue is, a generai Account of the Variety of the Foffil Bodies, both native and extraneous, reduced into 


\section{I N T R O D U C T I O N.}

fone Order under particular Heads, with Notice of their degrees of Rarity. If what is offered Sall afford any Information worthy the attention of the Lovers of Natural Hiffory, the Autbor will think bimfelf fufficiently rewarded for this Attempt of ex-. bibiting the Out-lines of a Work wobich merits the Abilities of a Perfon of more Fudgment and greater Leifure, to enlarge and perfect, than be pretends to be 10 Jiffed of. He cannot conclude bowever, witbout oblerving, that from the contemplation of $\int 0$ great a Veriety of extraneous Foffils difcovered in these Cliffs, wabich were evidently the produce of very different Climates, be thinks bimfelf rationally induced to believe, sibat notbing Sort of an univerjal Deluge could be a Caufe adegrate to jucb an Effect.

E. J. 


\section{FOSSILIA SHEPEIANA.}

$$
\text { NATIVE FOSSILS. }
$$

$$
\text { ThE C L I F F S. }
$$

T HESE are compofed of three different Strata 1 of brown Clay; the uppermoft is of the lighteft Colour, and moft friable; the loweft darkeft, and of a clofer Texture.

\section{P Y R I T 正.}

Coperas Stones, are the moft frequent of all tle Foffils of the Cliffs: They are of various Forms and Sizes; round, botryoide, flat, varying in every Digree, as well in Figure as Colour, and impregnating mort of the other Foffils found here.

\section{IUDUS HELMONTII:}

The WaxenVein; Nodules of which are lodged in the Cliffs in great Numbers; they are generally flat and oval, and of various Sizes, fometimes exceed. ing two Feet in length, all are covered with Coats 


\section{(136)}

in the Cliffs, but are frequently feen denudated on the Beach; the Tali are of different Sizes and Forms, generally they pais ftrait from one flat Surface of the Nodule to the other; in fome there is a double Set of them, rurning only half through the Body, with intermediate fparry Partitions; which being generally of a waxen Colour, at firft gave this Nodule its Name. The Number of the Tali in different Nodules are indeterminate; both the Tait and Septo vary in Colour in different Specimens - they are very common.

\section{BEZOAR MINERALE.}

Bodies, which are cruftated, ferrugineous, and of a rounded Form, covered with various Crufts of the Colour of the Clay of the Cliffs, and of different Sizes - Thefe are common.

\section{A L C U L I.}

Petbles. Amongt the numbers on the Beach, there have been fome Specimens collected, which by polining have proved very elegant.

\section{SELEN I T E S.}

Selenite, is found here in great Plenty, chiefly of the columnar fort, fome Specimens exceed fix Inches in length, but moft are of fhorter dimenfions, 


\section{( 137$)$}

frons, which are common. The rhomboidal Sort is not near fo common.

\section{LEPASTRUM. D. HILL.}

The Starry, Waxen Vein, is a Foffil peculiar to thefe Cliffs, not having as yet been difcovered elfewhere to the Author's Knowledge. Dr. Hill hath made from their Forms three Sorts of it, to which others might be added if nicely attended to. It is ever affixed to the Septa of the Ludus. Helmontii, where the Fiffures are fo far divided as to permit the Stars to hoot. The largeft specimens do not exceed three Inches in Length, and thefe are very rare; thofe are generally of a much lefs Size that are common.

\section{S U C C I N U M.}

Amber, is very uncommon; a few Specimens only, and thofe finall and tranfparent, are to be found here.

\section{EXTRANEOUS FOSSILS.}

$$
\text { V E GE T A B L ES. }
$$

\section{I N U M FOSSILE.}

Foffil Wood, in large Maffes, feemingly of Oals from its Grain, even to above three Feet in Length, 


\section{$\left(13^{8}\right)$}

is plentifully difperfed on the Beach, and generally impregnated with pyritical Matter.

\section{E QU I S E T U M.}

Horfe-tail, Joints of the naked Species, are fometimes found even to the Length of three Inches, but they are uncommon.

\section{F R U C T U S VARII.}

Fofjl Fruits, of very different Kinds, are here collected, in Variety and Number exceeding all that have been mentioned by Writers on this Branch of Natural Hiftory: Many of them have been drawn and compared with recent ones, in a particular Account given of them by the Author's late learned Friend, Dr. Fames Parfons, in the Philofophical Tranfactions - Vol. L. Part I. p. 396. Thefe have by fome been imagined mere Sports of Nature amongtt the Coperas; but as all the Specimens have not the misfortune to be impregnated by it, fome few, and thofe very elegant ones, being entirely free from it, and petrified, that Conceit without further Difcufion, muft fall to the Ground.

\section{A R I S T 死.}

Ears of Corn, fome fmall Species, are fometinses to be met with, imperfect and impregnated with the Pyrites, but they are not common. 


\section{( 139.$)$}

\section{Y C E T I T E.}

The Mufroom Stone. Here are two Kinds, the Conoide and Difcoide, both fmall, and the firft is by far the moft frequently to be found.

\section{A N I M A I U M PARTES. E L E P H A S.}

The Elepbant. A confiderable Number of the Bones of this very large Animal have been difcovered on the Eaftern Shore, a Mile from the Cliffs, on the ituthor's Manor of Nutts, in Laysciown; an Account of which may be feen in the Philofophical Tranfactions - Vol. xlviii. part ii. page $\sigma_{2} \sigma$.

$$
\text { T.E S T U D O. }
$$

The Tortoife. Two varieties have been found under the Cliffs, one of a more convexed Form, the other more depreffed; both are extreamly rạe.

\section{PIS C I U M P A R TES.}

The Heads of $F i$, from a very fmall Size to that of a middling Cod, are not unfrequent.

Palates of Fith, fome oval, others of a fquarifh form, when perfect, or nearly fo, are very uncommon; 


\section{(140)}

common; but fmall Portions of them, efpecially of the fquare Sort are common: To thefe laft, Mr. Llbuyd hath given the whimfical Name of Scopula littoralis, and to thore of the firft kind, Bufonita. In the Gentleman's Magazine for May, 1755, p. 409, is a Plate exhibiting both Kinds, in fine Prefervation.

Teeth of Fijh, ufually called Sbarks Teeth, are plentifully met with, except thofe of a large Size, which are very uncommon.

Back Bones of Fi $/ h$, are very common in feparate Joints, but with three or more united, are uncommon.

Parts of the Bodies of Fijh, with the Scales on them, are not very uncommon, but none entire have come to the Author's Knowledge.

Tails of Fifh, are frequently collected, and fome few with a Vertebra united to them; there are very few of a large Size.

\section{A NCRI MACROURI.}

Lobfters, are not uncommon, but none of the Specimens are perfect; the Bodies and Tails united are not fo frequent as when feparated; the Claws are alfo found feparately of various Sizes, but generally much injured.

Prawns 


\section{( 14 I )}

Prawns, are very rare; they are nearly perfect, and confiderably larger than thofe taken recent on the oppofite Coart of the Ine of Tbanet.

\section{A NCRI BRACHYURI.}

Foffil Crabs. Before the Publication of Dr. Hill's Hiftory of Folfls, thefe were extreamly rare in England; but fince that Time, thoufands of Specimens here collected, have paffed through the Author's Hands. There are two or three Varieties, but all common except very fair Specimens, which are ftill very rare; their Size varies from that of a fmall Bean, to that of the common Crab of the Coaft; fome of them, efpecially the fmaller ones, are impregnated by the Pyrites.

\section{E C H I N I T E.}

Eckinites, here are the Ovarius, Galeatus, Pileatus, Cordatus, and Placentiformis; but all of them are very rare, efpecially the laft.

The Spines, are of the foliated Kind, and that Sort named by Mr. Llbuyd, Volvola - both are rare.

$$
\text { A S T E R I A S. }
$$

The Star Fifh of five Ray's, has not yet been found entire, and its fingle Rays are uncommon. 


\section{$(142)$}

\section{ASTROITÆ ET ASTEROPODIUM.}

Star Stones, with depreffed Rays, and the Roots of them are very uncommon; the Specimens of the latter Kind were fo imperfect, that without better Information the Author was at a Lofs how to place them in his Collection.

\section{B E L E M N ITES.}

Belemnite, only one Specimen, and that imperfect, hath hitherto been difcovered.

\section{O C H Y IA.}

\section{T UBUL I MARINI.}

Worm Sbells, of various contorted Forms, gene. rally of a fmall Size, fome with their Shells, others denudated, frequently impregnated with the Coperas, are common.

\section{LAPIS SYRINGOIDES.}

The piped Waxen Vein, externally appears like the Ludus Helmontii, being found in incrufted Nudoles; they are evidently Oak that had firt been eroded by Sea Worrns, and afterwards their Per. forations flled with Pyritical Matter and Spar, 


\section{(143)}

fimilar to the Waxen Vein, furrounding each Perforation; thefe are very common.

\section{CONCHYLIA UNIVALVIA. \\ D E N T A L I A.}

The Tooth Sbells, generally finall, about an Inch long, curving towards the Apex in the more perfect Specimens, fome covered with their Shells, and others denudated; both Sorts are common.

\section{PATE L L A.}

The Limpet, is a very rare Fofrll, having oniy once been collected; the Specimen was fimall, of the ftriated Sort, and fomewhat imperfect.

\section{N A U T I L U S.}

The cbambered Nautilus, is a common Fonil of thefe Cliffs. Thefe vary in Size from that of HazelNut to that of a Man's Head, moft commonly they have their pearly Shell, and are often immerfed in Nodules; there are alfo fome fmall ones found of a ferrugineous Colour; very few of the large ones are perfect, having received Injury by compreffion.

OR'THO- 


\section{(144)}

\section{OR THOCERAT ITES.}

Small Fragments feemingly of this Genus are found here, but very uncommon; the Chambers or Partitions appear to be united by a Dove-tail Suture.

\section{TUBULI CONCAMERA'TI.}

Cylinarical Bodies, with feveral Concamerations paffing tranfverny through them, are generally of the Size of a Swan's Quill, and about two or three Inches in Length, fome petrified, others wholly pyritical, and are not uncommon.

\section{O C H L E $Æ$.}

Snail Shells, with a frort as well as with a produced Clavicle, fome with their Shells, others denudated, are generally of a fmall Size, and impregnated by the Pyrites, and are uncommon.

\section{T R O C H I.}

The Topßsells, are of different Forms, fome flatter, others more elevated, of various Sizes, fome with Shells on, others denudated, moft are impregnate with Pyrites, and are common. 


\section{( 145 , \\ CONCHA VENERIS.}

The Cowry. This is fmall, denudated, pyritical, and extremely rare.

\section{B U C C I N A.}

Wbilkes, are found in confiderable Quantities, varying in Form and Size, but generally fmall.

The Opercula are fometimes, though rarely feen.

\section{NER I T Æ.}

The Nerites, are common; and generally impregnated with Pyrites.

\section{T U R B I N E S,}

The Screw Sbells, and cafts of them without th. Shell, of various Sizes, and moftly impregnated? with Pyrites, are very common.

- CONCHYLIA BIVALVIA.

$$
\text { O S T R E A. }
$$

Folfil Oyfters; generally of a fmall Size, ar found here, but very uncommon; although t'le whole Coaft is covered with recent ones.

\section{$\mathrm{O}$ \\ CONCHT}




\section{$(146)$}

\section{CONCH青A NOMI}

These Sbells in great variety, tho' generally of a fmall Size, and impregnated with the Pyrites,-are very common.

\section{PECTUNCULI.}

Cockles, fmooth, ftriated, and fafciated, of a finall Size, are found in tolerable Plenty; but fair and large Specimens are uncommon.

$$
\text { T E L L I N Æ. }
$$

The Wedge Sbell. Small Specimens of which, generally denudated and pyritical, are very common.

$$
\text { M U S C U L I. }
$$

Mufcles. Thefe are extreamly rare, a few large Specimens, and one only of the common Mufcle have been hitherto met with; although the Coaft abounds with the latter.

\section{P I N $\mathrm{N}$.}

The Sea Wing. Only fmall Fragments of this Shell have as yet been difcovered, and thefe are very rare.

F. I N I S. 


\section{I $\quad \mathrm{N} \quad \mathrm{D} \quad \mathrm{E} \quad \mathrm{X}$ \\ OF T H E}

\section{E N G L I S H N A M ES.}

A.

Abele Tree

Adder's Tongue

Agrimony

Dutch, or common Hemp

Alder, common

black Berry-bearing

Alexanders

Alkanet, evergreen

baftard

Ale-hoof

All-good

All-heal, Clowns

narrow leaved

All-feed, upright Blite

leaft Rupture-wort

Anemony Wood

Angelica, wild

Apple-Thorn

Archangel, red

yellow

white
Page

87

73

2

36

3

39

108

15

16

I7

14

106

106

25

92

6

6

I 88

53

54

52 
ii

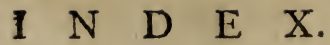

Arfinart, common

dead or fpotted

greater, with pale leaves

with fpotted ftalks

willow-leaved, perennial

Page

Afh Tree

82

81

82

3 I

82

39

mountain

110

Afh-weed

Afp, or Afpen

Avens, common

water

6

87

2 I

22

\section{B.}

$\mathrm{B}^{\prime} \cdot \mathrm{m}$, garden

67

Bafil, wild

Bafe Rocket

95

Batchelor's-buttons

Bear's-foot

Bedftraw, Ladies-yellow

Beech Tree

Beet, fea

Bell-flower, leffer round leaved

Berberry Bufh

Betony, water

wood

Birỏweed, black

12

103

12

37

28

great

finall

28

fea

28

Pirch Tree

13

Bird's Foot 


\section{N D E X.}

iii

Bird's Neft, or Wild Carrot

Page

Bitter-fweet

32

Blackberry

Blite, late flowering, or Fig.leaved the leart

maple leaved

upright

round-leaved, or All-feed

fea

Blood. wort

IOS

98

13

14

26

26

25

14

55

15

3 工

64 white

Box Tree

Brakes, common

Bramble, or Briar, common

fmall

Briar, fweet

Briony, blark white

17

$3^{8}$

98

$9^{8}$

97

112

15

40

78

120

1:0

middle, long leaved narrow leaved

120

67

96

96

fea

Buckbean

Buck's-horn plantain

84

Buck-wheat, or Brank

Bugle

Buglofs, fmall wild

Englih vipers 
I $N$ D $\quad E \quad X$.

Bullaee Tree, black

Page

white

90

Burdock

with fmaller heads

taller, with a larger fower

90

$5^{6}$

56

with wooly heads

Burnet

Burnet-faxifrage, great

leffer round leaved fmall

Bur-reed, great branched not branched

Bufh Vetch

122

Butcher's Broom

99

Burter-bur, common

82

Butter-cups

93

c.

Cabbage, fea

I5

Calamirt, common

18

Calts-inout

Caltrops water, greater leffer

88

88

Cammock

Camomile, fweet-fcented creeping Campion-bladder

corn, white

greater corn red Alowered

wild white

Dover

7

25

64

64

$6+$

64

64

62

fea

62

narrow leaved

63

Can- 


\section{N D E X:}

Canterbury Bells

Page

Cairo!, wild

18

Cat-rnint

Cat's-tail, great

32

118 leffer

Celandine, greater

118 leffer

Centory, leffer

$\begin{array}{ll} & 24 \\ \text { leffer, with a white Flower } & 24\end{array}$

Charlock, white yellow

or white Muftard

Cheefe-rening yellow

79

25

23

96

95

95

40

Chier, wild

Cherry wild, common

Cherry-wood

Chervil, fmall Hemlock wild

Chefnut Tree

Chick-weed, breakftone

leaft

marfh, great moufe-ear, broader leaved

59

24

73

71

24

23

5

4

4

3

24

fea leaft

purple flowered

ftar-headed

Chickweed or Speedwell

Cicely, wild

Cinque-foil, common

Tormenti] narrow leaved 
vi

\section{N D E X.}

Ciftus, dwarf, or little Sun-flower

Page

Clary, common Englinh wild

Climber, great wild

44

Cleavers

Clot-bur

with fmaller heads

taller, with a purple flower

47

27

with wooly heads

Clover, Dutch

Clown's All-heal

Cockle

Cock's comb, great marh, red rattled red rattle

Cock's-head yellow

Codlings and Cream

Colewort, fea

Colt's-foot, common

Columbines

Comfrey

Corn, bottles

Feverfew
Marygold
Mint
Parney, fmall
Sallad
$\quad$ late flowering
fmall

Cornel, female

Cotton Thiftle

Cow's Lung-wort

Cow Parfnep

Cownip, great 


\section{I $\mathrm{N} \quad \mathrm{D} \quad \mathrm{E} \quad \mathrm{X}$.}

vii

Cow.weed

Page

Cow-wheat, common, wild

Crab Tree

Crane's-bill, dove's-foot

field

hemlock leaved

jagged leaved

long ftalked

fmall Alowered

fea

Creffes, water

\section{winter, or Rocket}

Crimfon Grais Vetch

Crofs-wort

Crowfoot, common creeping corn

fineft leaved

ivy leaved

round, rooted, or bulbous

fweet-wood

water fennel leaved

fine leaved

round leaved

various leaved

Crow-garlick

upright meadow

Cuckow flower

67

66

$4 \mathrm{I}$

4 I

$4 \mathrm{I}$

42

42

42

43

107

35

23

30

94

93

92

93

93

94

92

93

94

93

94

3

63

Cudweed, black headed $\quad 43$

common 43

leait

long leaved, low branched

43

43

upright

43 
viii I N D E X.

Currants, black red

Page

$9^{6}$

97

D.

Daffodil, wild Englin

Daifie, common

$7 \mathrm{I}$

greater

12

58

Dandelion

Deptford Pink

Pandelion Hawkweed 32

32
32

22

Devil's-bit

102

yellow

guts
Dewberry
Dittander

Dittander

Dock, common broad leaved

great water

greener leaved

red leaved

3 I

98

fiddle

Tharp pointed

leffer

with curled leaves

Dodder

Dogberry

Dog's Mercury

Rofe, red flowered white flowered

Violet

Dove's-foot, common

Dropwort 


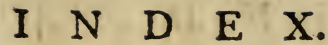

ix

Dropwort Hemlock

Page

Duck's-meat

$$
\text { water }
$$

73

73

58

58

greater

Dwale ivy leaved

$5^{8}$

12

301

$4 \sqrt{1}$

E.

Earth-nut

16

Eglantine

Elder, common

97

Dwarf

100

101

$$
\text { water }
$$

Elecampane

Elm, broad leaved common

fmooth-leaved, or Wych

73

44

125

125

125

Enchanter's Night hade

\section{F.}

Fennel, common

Hog, or Sulphur-wort

Fern, common male female, or common brakes fmall branched ftone

Feverfew

corn

field

$3^{8}$

38

$3^{8}$

$3^{8}$

$3^{8}$

67

25

25

Figwort, 


\section{I $\mathrm{N} \quad \mathrm{D}: \mathrm{E} \quad \mathrm{X}$,}

Figwort, common knobby rooted water

Finkle

Five leaved grafs

Flax, purging or wild dwarf perennial blue

Fleabane, middle

Flix-weed

blue flowered

Flower-de-luce, yellow water

Fluellin, round leaved

Fool's Parlley fharp pointed

Foxglove, purple

French Mercury, male and female

Frog-bit

Fumitory

Furze

$$
\text { great white }
$$

needle

\section{G.}

Gentian, dwarf autumnal

Germander-fpeedwell, or Chickweed wild

Gilli-flower, fea

Gladdon, ftinking 


\section{I $N \quad D E N$.}

$\mathbf{x i}$

Glafs-wort, white

Page jointed

I 4

5 I

prickly

Goat's-beard, purple

5 I

Goofeberry yellow

114

114

Gold of Pleafure

44

126

Goldilocks

Golden rod, common

94

I 24

Goofe-foot, common

I 4

Goofe-grafs

Tharp leaved

14

marn

Goofe Tongue

Gorfe

Goule

Grafs-vetch, crimfon

Green-wood

Go to bed at noon

Gout-weed

Gromwell, or Gromile

Ground Ivy

upright

Groundfel, common

mountain

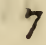

7

91

41

40

23

$4 I$

I 14

6

6 I

37

306

105

127

H.

Hare bells

Hare's ear, the leaft

Hare's foot

Hart's tongue

Hawk nut, or Hog-nut

48

16

115

83

16

Hawk- 
Hawkweed, with bitten roots

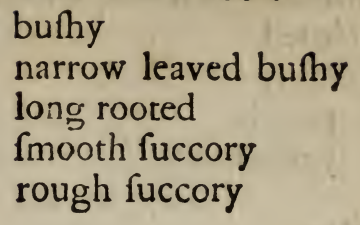

Hawthorn

Hazel-nut Tree

Three faces under a hood

Heath common, or Ling crofs leaved

fine leaved

Pea, or wood pea

Hedge muftard

parfey

Hellebore, great baftard black

Hell-weed

Hemlock Chervil, fmall with rough leaves

71

dropwort

leffer, or Fool's parley

water

Hemp-agrimony, common or Dutch

whole leaved

Henbane, common 


\section{$I N D E X$.}

Hep-tree

Page

Herb, Bennet, or common Avens

Gerard

Paris

Robert

47

2 I

Two-pence

High-tawer

Hog-fennel

Hog-nut

Holly-tree

42

72

119

83

נ 6

Hone-wort

Honeyfuckle, common trefoil

Hop, male and female trefoil

Horehound, ftinking

water

white

Horn-beam Tree, or Horfe-beech

Horfe-radifh

Horfe-tail, corn

leffer, marh

river

wood

Horfe-fhoe Vetch

Hound's-tongue, great

Houfe-leek leffer green leaved

Hyacinth, Engling

Jack-by-the-hedge Iron-wort 
xiv $\quad$ I $N \quad D \quad E \quad X$

Ivy, climbing or berried

Page

Juniper, common

K.

Kidney-vetch

wort

124

29

Knapweed, common

great

50

Knawel, annual

52

Knee-holly

Knot-grafs, common

German

oblong narrow leaved

52

fmall narrow leaved

L.

Ladies Bediftraw, mountain

70 white

Smock yellow

Finger fmall flowered

Thiftle

20

20

77

Traces, triple

Lamb's lettuce

late flowering

I ang-de-beuf

Larkfpur, wild

l averder, fea

Laurel, dwarf 


\section{I $N \quad D \quad E \quad X$.}

Lettuce, Ivy leaved wild

Page leaft wild

Lilly-convally

water wbite

Ling

$$
\text { yellow }
$$

72

Live-long

Lords and Ladies

Loofe-ftrife, greater fmooth-leaved

leaft fmooth-leaved codded

leffer hairy codded with fmall flowers

middle fmooth-leaved

purple fpiked

35

yellow

Loufewort, common marfh, red rattle, Cock's-comb 80

Liquorice, wild or Yetch

\section{M.}

Madder, little field

$$
\text { great baftard }
$$

fmall mountain

wild, or great baftard

Madwort, German

Maiden hair, white

$$
\text { common black }
$$

Mallow, common

$$
\text { fmall wild, or dwarf }
$$

$$
\text { marih }
$$

Maple, common

$$
\text { greater, or Sycamore }
$$


xvi $\quad I \quad N \quad D \quad E \quad X$.

Mare's-tail

Marigold, corn

$$
\text { marfh }
$$

Page

garden

126

Marjoram, pot

wild

Matfellon, common

50

May Lilly great

50

59

weed, ftinking

Meadow Pink

Rue

Sweet

Medick-Vetchling

Melilot

$$
\text { trefoil }
$$

Mercury, common Englin dog's

French, male and female

Milfoil

water, feathered or fpiked verticillated

Milk-wort

fea

Mill-mountain

Mint, Nep or Nap

long leaved horfe round leaved horfe

fpear

water

with whorled coronets 


\section{N D E X.}

Mofcatel, tuberous

Mother-wort

Mother of thyme, common

Mountain Afh

Moufe-ear fcorpion grafs common creeping

Mug-weed water fcorpion grafs

Mug-wort

Mullein, great white hoary or white

Murk Thirtle fage leaved black

Muftard, common hedge tower, leffer white

Myrtle, Dutch

\section{N.}

Navel.wort

Navew, wild

Needle Furze

Nep or Nap, or Cat-Mint

Nettle, dead hedge hemp-leaved, with a parti-coloured flower

red yellow ftinging, common white flowered leffer 
xviii I $N$ D $E$ E $X$.

Nettle-hemp, white flowered

Page

fair flowered

\section{yellow}

Nighthade, common

Enchanter's

woody

12

27

108

Nipple-wort

Nofe-bleed

Nut-earth, Kipper, Pig, Hawk or Hog

Oak Tree, common

91 with acorns on Thort.foot ftalks 9 I

Oculus Chrifti, or Chrift's eye

One Berry

45

Orach, common wild

grafs leaved

10

narrow leaved wild

with a fpear pointed leaf

IO, I I

jagged fea

wild fea narrow leaved

wild fea

10

ftinking

Orchis, female

male, or male fool's ftones

butterffy -

$7+$

bee

bee hairy, or humble bee

74

fly

frog

75

76

Orchis, 


\section{N D E X:}

xix

Page

Orchis, man green

74

purple

75

Neapolitan

little purple flowered

74

$7^{5}$

purple late flowerng

76

yellow or murk

bird's neft

75

ladies traces

Orpine

77

Ofier

Ox-eye

lips

tongue

Pagils, common

Panfies, two faces under a hood three faces under a hood

Park-leaves

Parley, garden

corn or Honewort

82

corn fmall

107

23

hedge

knotted

23

ftone baftard

Parfnep, Cow

127

107

great water

bI I

leaft water

107

308

water creeping.

wild

108

79

12

Pea,

\section{Paul's Betony}

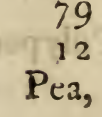


Pea, everlafting broad leaved wood or heath

\section{narrow leaved}

Pear, wild

Pellitory of the wall baftard

Penny royal

Penny-wort, marh wall

Pepper-wort

Pepper, water

Periwinkle, the greater the leffer

Peftilent Wort

Petty Whin

Pig-nut or Hog-nut

Pilewort

Pimpernel, male

$$
\text { yellow }
$$

Page

Pin Pearl-wort

Pink, Deptford meadow

Pipperidge Bufh

Plantain, Buck's-horn

great

hoary

ribwort

fea

narrow leaved fea

water great

ftar-headed

Polypody 


\section{N D E X.}

$x x i$

Page

89

fine or fennel leaved $\quad 88$

grafs leaved

horned

88

perfoliated

fea

126

89

88

87

87

87

trembling

Poppy, Corn

79

red

fpatling

yellow horned

Plowman's fpikenard

Prick-madam

Prick-wood, Cornel-tree

Spindie tree

Primrofe, common

79
64

78

Privet or Prin

Pudding-grafs

Purnane, water

fea

Purfley Piert

$8 \mathrm{I}$

Q.

Quick

Quicken Tree

R.

Radifh, Horfe

Water

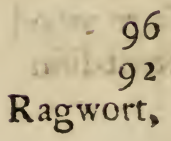


Ragwort, broad leaved marfh

Page $5^{\circ}$ common hoary perennial with Groundfel

Ramfons leaves

Rafpberry

Rattle, yellow red marfh

Reed Mace

$$
\text { leffer }
$$

Reft-harrow, Corn fpinous

Ribwort Plantain creeping

Roan Tree

Rocket, or Winter Creffes

$$
\text { bafe }
$$

water

fea

Rofe-bay, Willow herb

Rofe, Burnet dog's red flowered white flowered

Rue, meadow wall

whitlow grafs

Rupture-wort, the leaft 50 50 50

S.

Sage wood

Saint-foin 


\section{N D E X.}

Saint John's-wort

Saint Peter's-wort

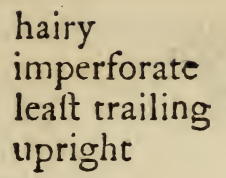

Sallow, common round leaved

Salt-wort, annual

black

Sampire

$$
\text { golden }
$$

marnh

perennial

Sanicle

Satyrion Royal, male handed

Sauce alone female handed

Saw-wort

Saxifrage, common golden meadow

white

Rue-leaved

Scabious, common field leffer

Sheep's hairy

Scad Tree, or Scad Plumb Scorpion-grafs

water

Sea Bindweed

Buckthorn or fallow

Cabbage

Campion

Colewort

Pond-weed

xxiii

Page

48

48

49

49 .

49

49

100

99

43

30

9

99

51

101

76

76

46

105

10 I

105

102.

102

102

102

95

90

70

71

28

96

15

62

326

88

Sea 
xxiv $\quad$ I $N$ D $\quad$ E $\quad$ X.

Sea Hoily

Heath finooth

Gilly-fower

Rocket

Lavender

Plantain

narrow leaved

Spurrey, fmall flowered

Starwort

Scurvy-grals

Self-heal

Sengreen, white

common

Septfoil

Service Tree, common

Setter-wort

Shepherd's Needle

Purfe

Rod

Silver Weed

Simfon

Sloe Tree

Smallage

Snap-dragon, greater

Sneeze-wort

90

Snow-drop

Soap-wort, common

Sorb Tree

Sorrel, common

Wood

Sow-bane 


\section{$\begin{array}{lllllll}I & N & D & E & X . & \quad & x x v\end{array}$}

Sow-thiftle, common

Page

prickly with jagged leaves $\quad 109$ with lefs jagged leaves 109 finooth with lefs jagged leaves 109 Tree

Spear-thiftle

Spear-wort, great lefrer

Szpeedwell, or Chickweed

Germander

110

19

94

$9+$

12 I

ivy-leaved

little fmooth

12 I

i2 I

male

water, narrow leaved

$12 \mathrm{I}$

12 I

with long leaves 120

Spikenard, Plowman's

Spatling Poppy

Spindle 'Tree

Spleenwort, or Miltwafte

rough

Spurge, broad leaved

dwarf, fmall annual

Laurel

petty

Sun, or Wart wort

Wood

Spurrey, Corn

purple

fea

I I

64

36

9

$6 r$

114

113

57

113

113

113

4

II I

fmall flowered

Squinancy wort

Star-headed $\mathrm{W}$ ater Chickweed

Star of the Earth

Thiftle 
$\begin{array}{llllll}\text { xxvi } & \text { I } & \text { N } & \text { D } & \text { E } & \text { X. }\end{array}$

Star-wort, autumnal

vernal

Page

fea

Stich-wort, greater

leaft

22

leffer

middle, a variety

Stonecrop, yellow

Wall Pepper

yellow reflexed

mountain

22

22

infipid

Stone Parney, baftard

104

104

104

103

104

Strangle Tare

122

Strawberry

barren

trefoil

Succory, wild

Sulphur-wort

Sun Hower, little

Sweet Briar

Swine's Crefs

Sycamore

\section{T.}

Tanfey, common wild

Tare, common everlafting hairy fmall wild fmooth 1:2 80 122

fpring 


\section{I $\mathrm{N} \quad \mathrm{D} \quad \mathrm{E} \quad \mathrm{X}$.}

Tare, ftrangle

Page tine

with fmooth pods

Teafel, wild

fmall wild

Tent-wort

99

Thiftle, conmon way, or creeping

Carline, dwarf

$2 L$

19 wild

2.1

Cotton

21

Marßh

20

Milk, or Lady's

20

without fpots

Mufk

20

20

Spear

Star

up $n$ Thifte

Wel:ed, with Imall flowers

Thorn Appie

Thorn, black

white

common purging

fea buck

Thorough wax

Three faces under a hood

Thrift

Throat-wort, great

Thyme, mother of

$$
\text { Lemon }
$$

Tine Tare

$$
\text { with fmooth pods }
$$

Toad Flax, common yellow

ic

21

$1 \hat{y}$

20

104

90

60

9)

96

36

124

11 I

18

105

105

30

30

60 the lealt

Tooth-wort 


\section{xxviii I $N \quad D \quad E \quad X$.}

Tormentil

\section{Cinquefoil} creeping

Tower Muftard, hairy

Traveller's Joy

Tree, pliant mealy Trefoil, Bird's foot

Page

114 80

114

117

27

122

62

greater

common purple

61

Hare's-foot

Heart

Hedge-hog

Hop

leffer

long leaved purple

Mlanh

Melilot

oval headed

Teafel headed

finall

Strawberry

white flowered meadow

Triple Ladies Traces

i 5

115

116

116

117

'Tutfan

'Turnep

hairy

Two faces under a hood

Valerian, great wild V. jagged leaved fmall wild or mar?h 


\section{I $N D E$ D.}

xxix

Venus-comb

Vervain

mallow

Page

103

i 19

2

Vetch Bufh

Crimfon grafs

122

common

23

122

tufted

wild

30

fpring

tufted Horfe thoe

Kidney, or I_ady's finger

Vetchling, common yellow baftard Medick

Violet, Dog's or wild

purple fweet

Throat wort leaved

white

water

hairy

Viper's Buglofs

Englifh

122

122

37

124

57

73

I 23

124

123

123

47

123

33

33

W.

Wake Robin

8

Wall-fower

Penny-wort

Pepper

29

Rue

104

wort

Wart-wort

Water Betony

Caltrups, greater

99

IOI

113

103

88

88

Water 
$\begin{array}{llllll}\operatorname{xxx} & \text { I } & \mathrm{N} & \mathrm{D} & \mathrm{E} & \mathrm{X}\end{array}$

Water Crow-foot, fennel leaved

Page

fine leaved

92

fineft leaved

93

Ivy leaved

92

round leaved

93

various leaved

Creffes

93

Dock, great

107

Dropwort

\section{Hemlock}

Elder

Figwort

79

73

73

73

Gladiole

Heınlock

Hemp agrimony, trifid

with an undivided

Horehound

leaf

Lilly, white

103

17

83

120

yellow

Mill-foil, feathered or fpiked verticillated

Mint

\section{hairy}

with whorled coronets

Parfnep, creeping

great

leaft

Pepper

Plantain, great

ftar-headed

Purflane

120

64

72

73

87

81

107

107

68

108

107

108

82

84

3 I

87

Ragwort

50 


\section{N D E X.}

Water Speedwell, middle long leaved

Page narrow leaved

Wort

Violet

Way-bread

Way-faring Tree

Weed, yellow

I 20

120

I I I

47

84

I 22

Whin

petty

White-glafswort

White-rot

White-thorn

Whitlow-grafs, common rue leaved

62

41

7

14

48

69

79

102

Williams, wild

Willow, common white

round eared Shining

63

99

100

fand

100

fweet

Willow-herb, purple fpiked great flowered

greater fmooth leaved

leaft fmooth leaved codded leffer hairy codded with fmall

flowers

middle fmooth leaved

Rofe bay

40

99

65

64

65

Winter Creffes yellow

Wood Anemone

Betony

12

bine

18 roof 
xxxii I N D E X.

Wood Pea

Page

Sorrel

$7^{8}$

waxen

Sage

Spurge

78

59

103

113

Wormwood, common white fea

Wort, white glafs

Wych Elm

Hazel

I

I

14

J 25

125

Y.

Yarrow, comnion

69

Yellow-weed, common

62

Yew Tree

112 


\section{N $\quad \mathrm{D} \quad \mathrm{E} \quad \mathrm{X}$}

O F T H E

LI N $N$ A $N$ NA MES.

A.

Acer campeftre

Pfeudo platenus

Achillea Millefolium

Ptarmica

Adoxa Mofchatellina

Aegopodium Podagraria

Aethufa Cynapiuns

Agrimonia Eupatoria Agroftemma Githago Ajuga reptans

Alifma Damafonium

Plantago

Allium vineale

Page

I

69

91

70

6

27

2

63

16

Urfinum

Althæa officinalis

Amarantus Blitum

Anagallis arvenfis

Anchufa fempervirens A nemone nemorefa 
xxxiv I $\mathrm{N}$ D $\mathrm{E} \quad \mathrm{X}$.

Anethum Fœniculum

Angelica fylveftris

Anthemis Cotula nobilis

Anthyllis Vulneraria

Antirrhinum minus

Elatine

Linaria

majus

fpurium

Aphanes arvenfis

Apium graveolens

Petrofelinum

Aquilegia vulgaris

Arctium Lappa $\beta . \gamma . \delta$.

Arenaria marina $\beta$.

marina $\gamma$.

rubra

peploides

ferpyllifolia

Ârtemifia Abfinthium

maritima

vulgaris

A rum maculatum

$\mathrm{x}$.

Page

38

6

24

25

124

127

60

60

7

60

8 I

8

82

8

$5^{6}, 57$

4

110

111

4

4

I

I

8

8

8

$9^{8}$

Cynanchica

Afplenium Adiantum nigrum

Ceterach

Ruta muraria

Scolopendrium

Trichomanes

Aftragalus glycyphyllos

After Tripolium 


\section{N D E X.}

XXXV

Atriplex haftata

haftata $\beta$.

laciniata

litoralis $\beta$.

litoralis

patula

ferrata

portula coides

Atropa Belladona

Page

10

I I

10

II

10

10

IO

II

12

B.

Ballota nigra

Bellis perennis

11

12

12

12

12

13

Alnus

Berberis vulgaris

Beta vulgaris

Betonica officinalis

Betula alba

Bidens tripartita

cernua

Borago officinalis

120

Braflica Napus

15

71

Rapa

oleracea

Blackitonia perfoliata

Bunium Bulbocaftanum

Bunias Cakile

Bupleurum rotundifolium

17

16

tenuiffimum

Butomus umbellatus

Buxus fempervirens 
xxxvi I N D E $\mathrm{X}$.

C.

Calendula officinalis

Page

Callitriche autumnalis

126

verna

Caltha paluftris

Campanula rotundifolia

Trachelium

Cardamine parvifora pratenfis

Carduus acanthoides

II I

11 I

86

18

18

19

18

20

acaulos

crifpus

19

Janceolatis

Marianus

19

19

Màrianus $\beta$.

20

20

nutans

paluftris

20

20

Carlina vulgaris

2 I

Carpinus Betulus

78

Caucalis Anthrifcus arvenfis

Centaurea Calcitrappa

Cyanus

Jacea

Scabiofa

Ceraftium aquaticum

femidecandritum

vifcofum

vulga:um

Ceratophyllum denierfum

23

23

21

31

50

50

4

24

3

3

126

Charophyllum fylveftre 
Cheiranthus Cheiri

Chelidonium majus

Glaucium

Chenopodium album

Bonus Henricus

hybricum

59

79

78

13

$1+$

26

maritimum

murale

polyfpermum

14

14

rubrum

ferotinum

Vulvaria

urbicum

Chryfanthemum Leucanthemum fegetum

Chryfofplenium oppofitifolium

Cichoreum Intybus

Circœa Lutetiana

Ciftus Helianthemum

Clematis Vitalba

Clinopodium vulgare

Cochlearia anglica

$$
\begin{aligned}
& \text { Armoracia } \\
& \text { Coronopus }
\end{aligned}
$$

Conium maculatum

Convallaria majalis

Convolvulus arvenfis

$$
\text { fepium }
$$

Soldanella

Conyza fquarrofa

25

14

13

13

26

58

26

IOI

26

27

44

27

28

28

$9^{6}$

71

26

59

28

28

28

II

Cornus fanguinea

Corylus Avellena

Cotyledon Umbilicus Veneris

Crambe maritima 
xxxviii I $N$ D

Cratægus Oxyacantha torminalis

Crepis tectorum biennis

Crithmum maritimum

cucubalus Behen vifcofus

Cufcuta europæa

Cynogloffum officinale officinale $\beta$.

D.

Daphne Laureola

Datura Stramonium

Daucus Carota

Deiph nium Confolida

Dianthus Armeria

Digitalis purpurea

Dipfacus pilofus

Draba verna

Echium anglicum vulgare

Epilobium anguftifolium

hiriutum

65

montanum

paluftre

$6_{4}$

65

ramofum

tetragonum 


\section{I $N \quad D \quad E \quad X$.}

Equifetum arvenfe

Page

fluviatile

33

paluftre

fylvaticum

Erica cinerea Tetralix vulgaris

Erigeron acre

Ervum hirfutum folonienfe tetrafpernum

Eryngium maritimum Eryfinum Alliaria Barbarea officinalis

Euonymus europæus Eupatorium cannabinum Euphorbia Amygdaloides exigua Heliofcopia Peplus platyphyllos

Euphrafia officinalis Odontites

Fagus Caftanea fylvaticus

Ficaria verna

Filago germanica montana

Fragraria fterilis vefca

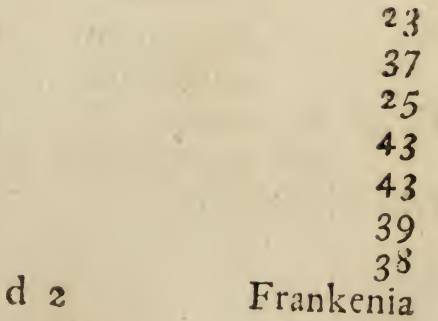


xl I $\quad$ N $D$ E $X$.

Frankenia lævis

Fraxinus excelfior

Fumaria officinalis

39

claviculata

G.

Galanthus nivalis

Galeopfis Ladanum

Tetrahit

Galeobdolon

53

53

Galium Aparine 54

uliginofum

verum

Moliugo

montanum

- paluftre

Genifta anglica

tinctoria

Gentiana Amarella

Centaurium

Geranium cicutarium

40

70

70

70

40

$4 I$

$4 \mathrm{I}$

24

4 I

42

columbinum

diffectum

42

molle

$4 \mathrm{I}$

pufillum

42

inaritimum

42

robertianum

Geum urbanum

42

$2 t$

22

rivale

43

Glaux maritima 


\section{$\begin{array}{lllll}\text { I } & N & D & E & X\end{array}$}

\section{xli \\ Page \\ 106 \\ 17 \\ 43 \\ 43}

Glechoma arvenfis

hederacea

Gnaphalium fylvaticum

$\mathrm{H}$.

Hedera Helix

Hedyfarum Onobrychis

Helleborus fœtidus viridis

Heracleum Spondylium

Hieracium Pilofella fabaudum umbellatum

Hippocrepis comofa

Hippophae Rhamnoides

Hippuris vulgaris

Hottonia paluftris

Humulus Lupulus

Hyacinthus non feriptus

Hydrocharis Morfus ranæ

Hydrocotyle vulgaris

Hyofcyamus niger

Hypericum Androfamum

hirfutum

humifufum

montanum

perforatum

pulchrum

44

73

45

44

I I I

83

46

46

37

96

59

47

62

48

II 2

48

48

49

48

49

49

48

49

quadrangulum

Hypochæris radicata 
xlii $\quad$ I $N \quad D \quad E \quad x$.

I.

Jafione montana

Ilex Aquifolium

Inula crithmoides

Page

dyfenterica

Helenium

Pulicaria

95

2

9

29

44

29

Juniperus communis

$5^{I}$

$5^{\text {I }}$

Pfeudacorus

Lacluca faligna

Lamium album

amplexicaule

rubrum

Lapfana communis

Lathræa fquamaria

Lathyrus latifolius

Niffolia

54
6

pratenfis

fylveftris

Iemna minor

$$
\text { polyrrhiza }
$$

trifulca

Leontodon autumnale

hifpidum

Taraxacun

Leonurus Cardiaca

Lepidium latifolium

L.jguftrum vulgare 


\section{N D E X.}

\section{xliii}

Page

Linum catharticum

perenne

Radiola

tenuifolium

Lithofpermum officinale arvenfe

Lithrum Salicaria

Lonicera Periclymenum

Lotus corniculata

Lychnis dioica

$$
\gamma_{0}
$$

$\beta$.

Flos cuculi

Iycopfis arvenfis

Lycopus europæus

Lyfimachia nemorum

tenella

vulgaris

Nummularia

of

$6 \mathrm{t}$

92

60

6 I

16

99

18

62

6 I

64

64

63

15

64

5

72

65

72

M.

Malva Alcea

rotundifolia

fylveftris

Marrubium vulgare

Matricaria Chamomilla. inodora

Parthenium

Medicago arabica

aribica $\gamma$.

lupulina

${ }^{2} 6$

66

Melampyrum fylvaticum 
xliv I N D E X.

Meliffa Calamintha officinalis

Mentha aquatica

Page

$\gamma$.

arvenfis

longifolia rotundifolia

fpicata

Pulegium

Menyanthes trifolia

Mercurialis annua

Myrica Gale perennis

Myofotis fcorpioides

$\delta$.

Myriophyllum fpicatum verticillatum

Myagrum fativum

\section{N.}

Narciffus Pfeudo-Narciffus

Nepeta Cataria

lutea

72

\section{o.}

Oenanthe crocata

Ononis arvenfis

spinofa 


\section{I $N \quad D \quad E \quad X$.}

Onopordum Acanthium

Ophiogloffum vulgatum

Ophrys Anthropophora

apifera

apifera $\beta$.

Monorchis

Nidus avis

mufcifera

ovata

fpiralis

Orchis bifolia

latifolia

mafcula

maculata

militaris

Morio

purpurea

pyramidalis

uftulata

Origanum onites

vulgare

Ornithopus perpufillus

$\begin{array}{cc} & 74 \\ & 77 \\ & 75 \\ & 74 \\ & 75 \\ & \\ & 13 \\ & 77 \\ & 74 \\ & 76 \\ & 75 \\ & 76 \\ & 74 \\ & 75 \\ & 75 \\ & 76 \\ & 76 \\ & 17 \\ & 77 \\ & 77 \\ & 78 \\ & 78 \\ & 61 \\ & 78\end{array}$

P.

Papaver Rhœas

Parietaria officinalis

79

Paris quadrifolia

79

Paftinaca fativa

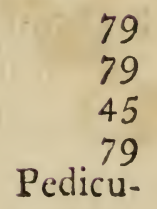


xlvi I N D E X.

Pedicularis paluftris fylvatica

Peplis Portulaca

Pteris aquilina aquilina $\beta$.

Peucedanum officinale

Phellandrium aquaticum

Picris Echioides

Pimpinella major

faxifraga

Plańtago Coronopus $\beta$. lanceolata Leoflingii major maritima media

Polygala vulgaris

Polygonum amphibium aviculare

Convolvulus B. et $\gamma$. Fagopyrum Hydropiper penfylvanicum

Perficaria

Polypodium Filix mas

\section{$\beta$.}

Populus alba vulgare nigra tremula 


\section{N D E X}

Potentilla Argentina argentea reptans

Potamogeton crifpum natans marinum gramineum pectinatum

- perfoliatum ferratum

Poterium Sanguiforba

Prenanthes muralis

Primula veris $\alpha$.

$$
\beta \text {. }
$$

Prunella vulgaris vulgaris

Prunus Avium infititia infititia $\beta$. et $\gamma$. fpinofa

Pyrus Malus communis

Quercus Robur

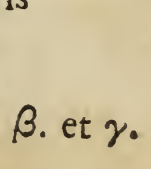

Q.

$$
\beta .
$$

\section{1 \\ 91}

R.

Ranunculus acris

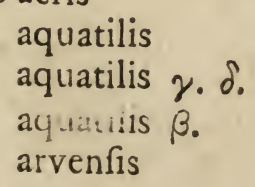


xlviii I $\quad \mathrm{N} \quad \mathrm{D} \quad \mathrm{E} \quad \mathrm{X}$.

Ranunculus Auricomus

bulbofus

Flammula

hederaceus

Lingua

repens

fceleratus

Raphanus Raphaniftrum

Refeda lutea

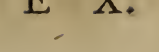

Iuteola

Rhamnus catharticus

Page

94

93

94

93

94

$-94$

94

96

$\beta$.

95

95

62

96

- Frangula

Rhinanthus Crifta galli

Rofa arvenfis

canina

97

Eglanteria

97

fpinofiffima

Ribes rubrum

nigrum

Uva crifpa

Rubus cæifus

fructicofus

Id aus

97

97

96

44

$9^{8}$

$9^{8}$

98

54

54

54

55

Britannicus

55
56

crifus

obtufifolius

pulcher

55

fanguineus

55

Rumicis acuti varietas 


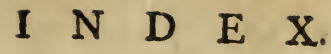

Rufcus aculeatus

$$
\begin{array}{r}
x \text { lix } \\
\text { Page } \\
99
\end{array}
$$

Sagina erecta

S.

Salix alba

procumbens

arenaria

amygdalina

capraa

vimenalis

Salicornia europæa $\beta$.

Salfola Kali

europæa

Salvia Verbenaca

Sambucus Ebulus

nigra

Samolus Valerandi

Sanicula europæa

Saponaria officinalis

Satyrium viride

Saxifraga trydactylites

granulata

Scabiofa arvenfis

Columbaria

Succifa

Scandix Anthrifcus

Pecten Veneris

Sedum acre

annuum

reflexum

reflexum $\beta$.

Telephium

fexangulare

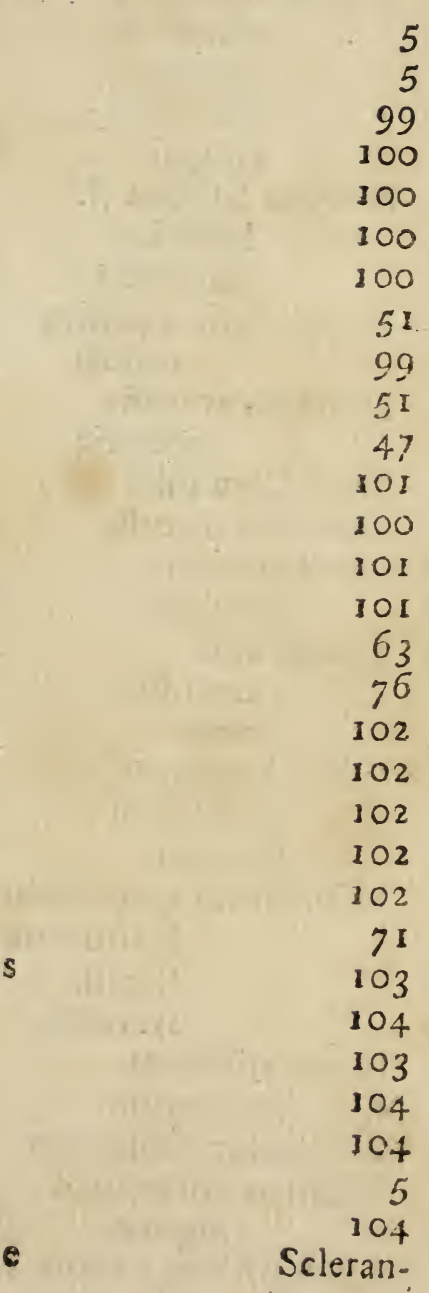


Scleranthus annuus Sempervivum tectorum Senecio aquaticus

erucifolius

Page $5^{2}$

Jacobæa fylvaticus vulgaris

Serapias latifolia $\beta$. latifolia 104 50 50 50 127 105 longifolia 45 45 45

Scrophularia aquatica 103 nodofa

Serratula arvenfis tinctoria

Séfeli Caruifolia

Sherardia arvenfis

Silene amœna conoidea

Sinapis alba arvenfis nigra I03 21 105 105 98 62 63 106

95 306

Sifon Amomum 107 inundatum 108 fegetum

Sifymbrium amphibium $\alpha$. 107

Nafturtium

Sophia

fylveftre

Sium latifolium nodiflorum

Sinyrnium Olufatrum 108

Solanum Dulcamara nigrum

Solidago Virga aurea 


\section{N D E X.}

Sonchus arvenfis

$$
\text { oleraceus } \alpha . \beta . \gamma . \delta \text {. }
$$

Sorbus aucuparia

Page

Sparganium ereetum $\beta$.

Spartium fcoparium

Spergula arvenfis

Spiræa Filipendula

Ulmaria

Stachys paluftris

$$
\text { fylvatica }
$$

110

109

110

I 10

40

4

37

125 106 40 22

22

22

Statice Armeria graminea $\beta$. Holoftea

111

Limonium

Symphytum officinale

\section{T.}

Tamus communis

Tanacetum vulgare

312

Taxus baccata

Teucrium Scorodonia

Thalictrum flavum

Thlafpi Burfa Paftoris

Thymus Acinos

Serpyllum

Serpyllum $\varepsilon_{\text {。 }}$

Tordylium nodofum

Tormentilla erecta

reptans

Tragopogon pratenfe porrifolium 
lii $\quad I N D E X$.

Trifolium agrarium

Page

arvenfe

filiforme

116

fragiferum

I 15

116

I 15

medium

Meliotus officinalis

117

maritimum

pratenfe

procumbens

repens

fcabrum

Tuffilago Farfara

Petafites

Turritis hirfuta

82

Typha latifolia

Valantia Cruciata

Valeriana dioica

officinalis

Locurta

Locuifta $\alpha$ :

Verbafcum Lychnitis nigrum

Thapfus

Verbena officinalis

Veronica agreftis

Anagallis

arvenfis

Becabunga

Chamædrys 


\section{N D E X.}

Jiii

Veronica officinalis

Page ferpyllifolia

12 I fcutellata

12 I

120

122

Opulus

73

30

Vicia Cracca

Lathyroides

122

fativa

122

fepium

122

27

123

123

123

124

123

123

124

Vifcum album .

\section{U:}

Ulex europæus

Ulmus campeftris

125

glabra; et $\beta$.

125

Urtica dioica 126 126 


\section{$\begin{array}{llllllll} & E & R & R & A & T & A\end{array}$}

The candid Reader is defired to correct thefe Errors and Omiffions.

\section{Page line}

420 after marina add $\beta$.

179 for Pluckley read Hedcorne.

2819 after Sandwich add and on Shellnefs.

364 after Sittingbourn add and on the Standard Key.

$42 \quad 25$ after Deal add and on Shellnefs.

4920 for eleganiflimum read elegantifimum.

- 22 for montenum read montanum.

5622 after 304 add $\gamma$.

572 after 304 add $\delta$.

595 for uncommon read common.

- 12 for common read uncommon.

734 for Fairbrook read Luddenham.

75 $2 \mathrm{I}$ afterplentifully add on Badgen Downs, Sparing?'.

8814 for granineum read gramineum.

14416 for uncommon read common.

Omitted to be inferted in their proper Places.

ANTIRRHINUM anguftifolium fylveftre.

R. S. ${ }^{*} 283$.

ANTIRRHINUM Orontium. H.F. 239.

'The leaft Snapdragon, or Calf's-\{nout.

In a Corn field at Luddenham - uncommor. July. A.

CASSIDA paluftris vulgatior flore cæruleo. R. S. 244.

SCUTELLARIA galericulata. H. F. 23 I. Hooded Willow-herb.

In a moift Hedge at Chartham Hatch-uncommon. Augutt. P. 

$5=-1$

. 

$x^{-1}-1$
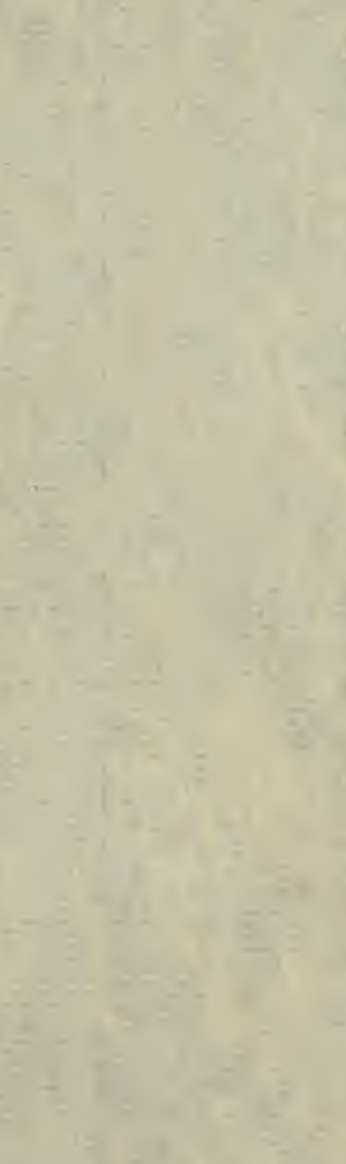


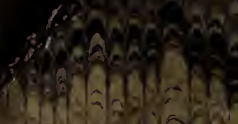

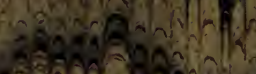

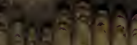

incon

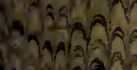

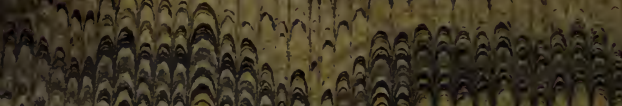

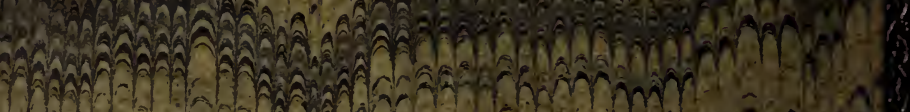

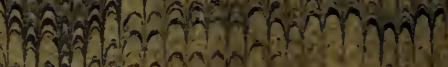

TANA

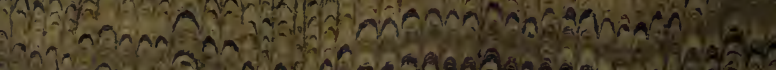
A.M드.

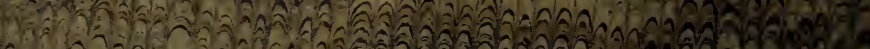

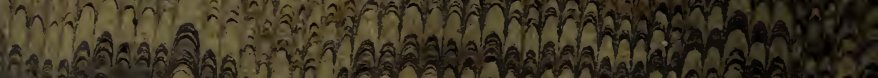

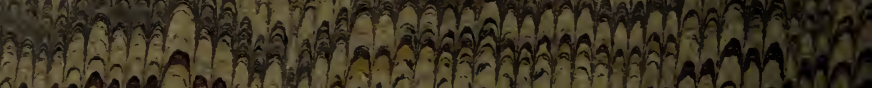

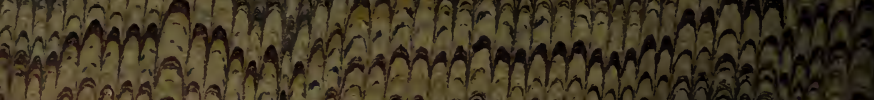

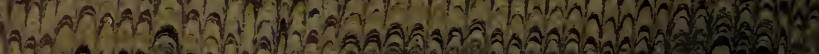

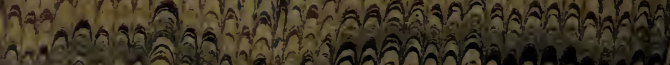

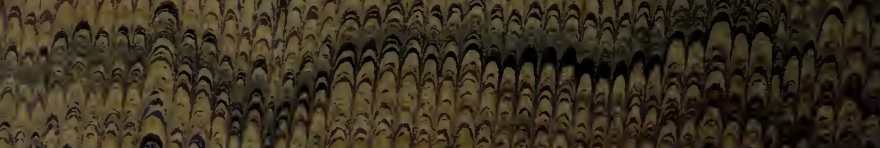

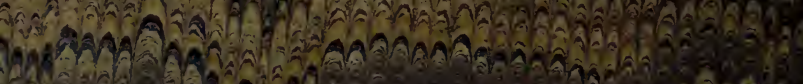

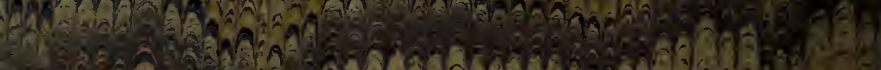

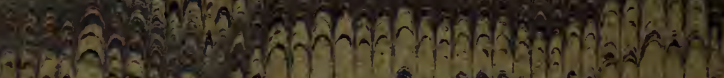
C.

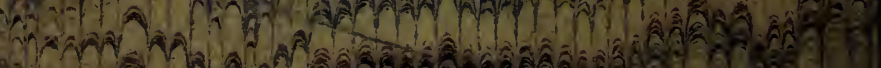

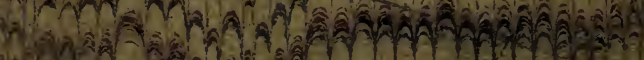

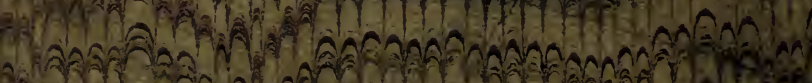

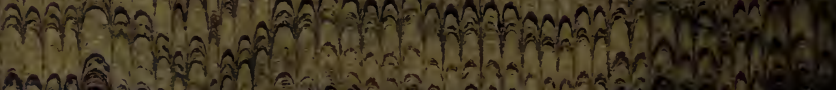

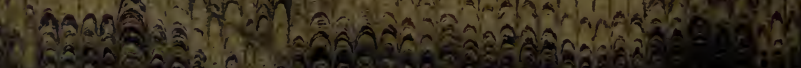

\section{Hansh Qhen}

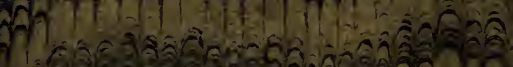

$$
\begin{aligned}
& \text { หลิ } \\
& \text { 6) Tha }
\end{aligned}
$$

